White paper

\title{
Equilibrium solubility measurement of ionizable drugs - consensus recommendations for improving data quality
}

\author{
Alex Avdeef, ${ }^{1,}{ }^{*}$ Elisabet Fuguet, ${ }^{2,3}$ Antonio Llinàs, ${ }^{4}$ Clara Ràfols, ${ }^{2}$ Elisabeth Bosch, ${ }^{2}$ \\ Gergely Völgyi, ${ }^{5}$ Tatjana Verbić, ${ }^{6}$ Elena Boldyreva ${ }^{7}$, Krisztina Takács-Novák ${ }^{5}$ \\ ${ }^{1}$ in-ADME Research, 1732 First Avenue, \#102, New York, NY 10128, USA \\ ${ }^{2}$ Departament de Química Analítica and Institut de Biomedicina (IBUB), Universitat de Barcelona, Martí i Franquès 1-11, E- \\ 08028 Barcelona, Spain \\ ${ }^{3}$ Serra-Húnter Program, Generalitat de Catalunya, Barcelona, Spain \\ ${ }^{4}$ RIA IMED DMPK, AstraZeneca R\&D, Gothenburg, Sweden \\ ${ }^{5}$ Semmelweis University, Dept. of Pharmaceutical Chemistry, H-1092 Budapest, Högyes E. u.9, Hungary \\ ${ }^{6}$ Faculty of Chemistry, University of Belgrade, Dept. of Analytical Chemistry, Studentski trg 12-16, Belgrade 11158, Serbia \\ ${ }^{7}$ Institute of Solid State Chemistry and Mechanochemistry SB RAS, Kutateladze, 18, Novosibirsk, 630128 Russia
}

*Corresponding Author: E-mail: Alex@in-ADME.com; Tel.: +1-646-678-5713

Received: May 08, 2016; Revised: June 21, 2016; Published: June 29, 2016

\begin{abstract}
This commentary addresses data quality in equilibrium solubility measurement in aqueous solution. Broadly discussed is the "gold standard" shake-flask (SF) method used to measure equilibrium solubility of ionizable drug-like molecules as a function of $\mathrm{pH}$. Many factors affecting the quality of the measurement are recognized. Case studies illustrating the analysis of both solution and solid state aspects of solubility measurement are presented. Coverage includes drug aggregation in solution (sub-micellar, micellar, complexation), use of mass spectrometry to assess aggregation in saturated solutions, solid state characterization (salts, polymorphs, cocrystals, polymorph creation by potentiometric method), solubility type (water, buffer, intrinsic), temperature, ionic strength, $\mathrm{pH}$ measurement, buffer issues, critical knowledge of the $p K_{a}$, equilibration time (stirring and sedimentation), separating solid from saturated solution, solution handling and adsorption to untreated surfaces, solubility units, and tabulation/graphic presentation of reported data. The goal is to present cohesive recommendations that could lead to better assay design, to result in improved quality of measurements, and to impart a deeper understanding of the underlying solution chemistry in suspensions of drug solids.
\end{abstract}

\section{Keywords}

shake-flask solubility; intrinsic solubility; water solubility; buffer solubility; thermodynamic solubility; Bjerrum curve; CheqSol; Potentiometric Cycling for Polymorph Creation; Henderson-Hasselbalch equation; aggregates; oligomers; micelles; hydrates; salts; polymorphs; cocrystals.

\section{Introduction}

Many investigational compounds in pharmaceutical development are practically insoluble solids consisting 
of ionizable molecules. Interpreting their solution properties in the course of measuring solubility - as a function of $\mathrm{pH}$ - can be problematic. Although there are a multitude of published methods, today's druglike molecules can pose special challenges, perhaps not adequately addressed in practice. The published literature is replete with incomplete/ambiguous and potentially misleading examples of solubility reports, frequently documented inconsistently. The solubility methodology appears to be in need of a timely tune up.

This commentary discusses ways to improve the measurement of thermodynamic solubility of druglike substances in aqueous media. (Topics such as solubility in bio-relevant media, kinetic solubility, and solubility of amorphous and of liquid-liquid phase separated (LLPS) solids are outside of its scope.) It is relatively scarce to find many high-quality solubility measurements that adequately cover the chemical space of drugs and research compounds. Measuring such data is expensive and analytical-resource consuming. Even with great expense, quality is not ensured when results are determined from under-designed assays. The "Solubility Challenge" from Llinàs et al. [1] and Hopfinger et al. [2] has spurred fresh discussion about data quality in solubility measurement in pharmaceutical research. Concomitantly, Baka et al. [3] examined the classical saturation shake-flask method, to assess which experimental conditions significantly affect the measured equilibrium solubility. More recently, solubility data quality has been reviewed by Avdeef [4].

Optimal experimental design to measure the aqueous equilibrium solubility of an ionizable substance requires a number of critical considerations. Briefly, the aqueous medium to which the substance is added ideally contains a buffer to help control the $\mathrm{pH}$. To this medium, the substance may be added in a number of ways: (a) as a highly supersaturated aqueous solution, (b) as a saturated hot solution, (c) as a concentrated solution in a water-miscible organic solvent, or (d) as a solid - which may or may not be in a thermodynamically stable form. The first three approaches are precipitation routes, while the fourth involves dissolution. The latter process is most commonly used, best understood, and is the backbone of the commentary here.

Solubility reactions, being heterogeneous, are often slow to reach equilibrium, especially in the case of lowsoluble substances. With sufficient added solid and enough time, chemical equilibrium is expected, where the substance in its stable crystalline form is in dynamic equilibrium with its solution form. As the dissolution proceeds, the solid phase can re-precipitate as another form with another structure (polymorphic transitions) and even another composition (formation of crystal solvates, salts, or loss of solvate from the starting solid). Dissolution of multi-component solids (salts - formed by cations and anions, or cocrystals - formed by neutral molecules, a drug and another molecule, termed a "coformer", which are often strongly hydrogen-bonded) adds complexity, since on their dissolution, re-crystallization of some components can start, depending on the solution conditions. The values of the solubility measured at different time points will therefore correspond to different solid phases, not necessarily the one initially introduced into solution. Sometimes a long equilibration time is needed to settle to the most stable crystalline form. In solution, the substance may undergo ionization, self-aggregation (forming water-soluble dimers, trimers, etc., or micelles in the case of amphiphilic substances), or complexation with some of the buffer components. In some cases, solids dissolve as clusters of molecules, which can be preserved for some time before being broken apart by the solvent. At true dynamic equilibrium, the forward (dissolution) and reverse (precipitation) reaction rates are equal to one another. In this dynamically equilibrated saturated solution, the concentration of the drug substance (summed over all of its dissolved forms) is the solubility. Both phases of the solubility process are important to consider, as 
measured solubility depends both on the solid and the solution forms. Since the solubility is temperature dependent, the temperature needs to be controlled. For ionizable substances, solubility depends on $\mathrm{pH}$, so the final (equilibrium) $\mathrm{pH}$ of the saturated solution, $\mathrm{pH}_{\text {sat }}$, needs to be measured directly.

Methods of characterizing the solid state (powder X-ray diffraction, differential scanning calorimetry, FT-IR reflectance/Raman spectra of slurries, elemental analysis, etc.) are well established. This is an important consideration with actionable consequences, since patents may be issued to cover particular solid state forms of a marketed drug substance.

However, characterizations of the solution state of ionizable substances of sparingly-soluble or amphiphilic substances that are surface active are far less well explored. This is especially true of ionizable substances in saturated solutions above their critical micelle concentration (CMC), with the assay temperature being above the Krafft point [5]. Under such circumstances, crystalline solids of the substance are effectively in equilibrium with micelles (or sub-micellar aggregates), in some cases leading to the transient formation of oils if the melting point of the neutral form of the substance is not far above the assay temperature. Such solutions can be very sensitive to changes in $\mathrm{pH}$ and/or temperature, as well as by the presence of background salts and other excipients [6].

This commentary is the outgrowth of discussions held during the "Thermodynamic Solubility Measurement of Practically Insoluble Ionizable Drugs - Case Studies \& Suggested Method Improvements" session at the $4^{\text {th }}$ World Conference on Physico Chemical Methods in Drug Discovery and Development (PCMDDD-4, http://www.iapchem.org/page.php?page id=56), held on Red Island, Croatia, 21-24 September 2015. The session focused on discussing the best practices for the saturation shake-flask measurement of solubility as a function of $\mathrm{pH}$ (the "gold standard" methodology), along with promising potentiometric methods, and attempted to reach a consensus about the best approaches for studying sparingly soluble drug substances. A questionnaire was prepared and distributed to the attendees. The responses received from several of the participants are summarized in Appendix D.

Several excellent books and reviews serve as background material for the commentary covering the solubility of druglike molecules: general coverage of solubility [7-10]; salt formation/selection [11-16]; polymorphism [17-20]; analysis of saturated solution speciation [21-27]; dissolution/solubility of cocrystals [28-37]; anhydrous forms versus hydrates [16, 38, 39].

\section{Case Studies}

The commentary begins with several selected case studies of molecules which illustrate appreciable challenges encountered in the measurement of solubility. Figure 1 shows four examples of drugs which reveal relatively simple logS-pH profiles, which can be more or less predicted by the Henderson-Hasselbalch $(\mathrm{HH})$ equation [27, 40,41]. Figure 2 shows three examples of molecules with logS-pH profiles that are consistent with the presence of anionic or uncharged self-associated aggregates, and one case consistent with drugbuffer interaction. Figure 3 illustrates the logS-pH profiles of haloperidol resulting from different salt precipitations, illustrating some degree of supersaturation for two cases near the Gibbs $\mathrm{p} K_{\mathrm{a}}[42,43]$ in the $\mathrm{pH}$ 4.1-4.9 interval and the possible formation of uncharged aggregates for $\mathrm{pH}>8$. Figures $\mathbf{4}$ and $\mathbf{5}$ ratchet up the complexity of logs-pH profiles of surface-active molecules which precipitate both as salts and as uncharged species. The examples were selected to show that care needs to be exercised in interpreting the shapes of the solubility-pH 
profiles [21-25] and that assays need to be optimized to enhance the confidence in the interpretations of the data.
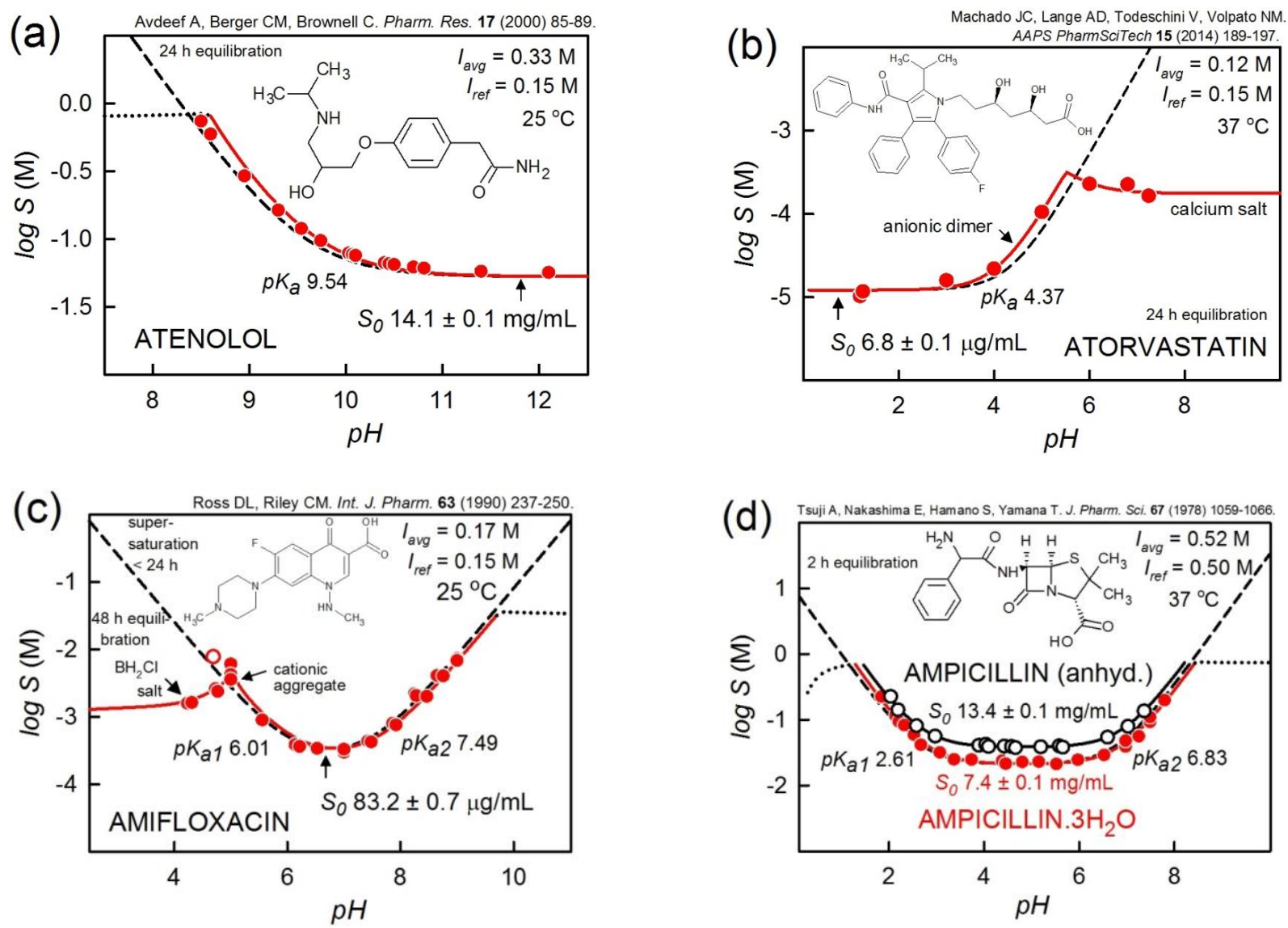

Figure 1. Four examples of drugs which reveal relatively simple logs-pH profiles, which can be more or less predicted by the Henderson-Hasselbalch equation.

The following logS-pH curve notations are used (except Figures $\mathbf{4} \mathbf{b}$ and $\mathbf{5 b}$ ):

- The dashed curves refer to the profiles calculated from the simple HH equation (not corrected for ionic strength or dilution effects)

- The solid curves are calculated using the refined constants from fitting the measured logS-pH data (filled circles) to the proposed equilibrium model, using the pDISOL-X program (in-ADME Research; www.in-adme.com/pdisol x.html)

- The dotted curves indicate regions of $\mathrm{pH}$ of subsaturation, where the substances are dissolved

- $S_{0}$ refers to the intrinsic solubility of the uncharged form of the substance

- $\mathrm{p} K_{\mathrm{sp}}$ refers to the negative log of the salt (or cocrystal, as in later sections) solubility product

- The reference and average ionic strengths are indicated as $I_{\text {ref }}$ and $l_{\text {avg, }}$, respectively. The former values correspond to the $\mathrm{p} K_{\mathrm{a}}$ determination conditions. Corrections are made for changes in the ionic strength in the solubility assays, as described by Völgyi et al. [22] and Wang et al. [44]

- The $j_{H}$ coefficients (Figure $\mathbf{3 b} \mathbf{b}, \mathbf{c}, \mathbf{d}$ ) refer to estimated pH electrode junction potential factors [45] in very acidic solutions $(\mathrm{pH}<1)$, as detailed in Appendix $\mathrm{C}$. 
Atenolol

Figure 1a shows the logS-pH profile of atenolol [46]. Since the weak base is relatively soluble, it takes a 3.8 $\mathrm{M}$ atenolol solution ( $1 \mathrm{~g}$ of the free base added to $1 \mathrm{~mL}$ of $50 \mathrm{mM}$ phosphate buffer) to span the concentration range shown in the figure. The ionic strength rises from 0.24 to $0.83 \mathrm{M}$ as $\mathrm{pH}$ is lowered from 9.5 to 8.5 using $1 \mathrm{M} \mathrm{HCl}$. Such pH adjustment is accompanied by a very large dilution effect. This requires that the $\mathrm{pH}$ electrode used be well-calibrated. Since the accurate $\mathrm{p} K_{\mathrm{a}}$ of atenolol is known, the fit can be further improved over what is shown, by assuming the presence of a neutral aggregate species, (Case 1b in [27]); the standard deviation drops from 0.047 to 0.015 log unit. The intrinsic solubility is slightly lessened from 14.1 to $11.0 \mathrm{mg} / \mathrm{mL}$. The performance of the $\mathrm{pH}$ electrode is quite important in this case study.

\section{Atorvastatin}

Figure $\mathbf{1 b}$ shows the logS-pH profile of atorvastatin added as the calcium salt [47]. This example illustrates the need to know the $\mathrm{p} K_{\mathrm{a}}$ accurately. The value of 4.37 at $37{ }^{\circ} \mathrm{C}$ was predicted [26] from the measured value at $25^{\circ} \mathrm{C}$ [48]. If 4.37 is the correct value, then the small parallel displacement of the solubility values in the $\mathrm{pH} 4-5$ region to lower $\mathrm{pH}$ is consistent with the presence of half-ionized aggregates, [AH.A $]_{n}$, (Case $3 a$ in [27]). On the other hand, it could be assumed that the reported $\mathrm{p} K_{\mathrm{a}}$ is inaccurate, and that no aggregates form in the $\mathrm{pH}$ 4-5 region. Then, the ionization constant determined by $p$ DISOL- $X$ would be $3.94 \pm 0.09$, using the $\log S-\mathrm{pH}$ data, based on methods popularized in older literature $[49,50]$. Such a big difference $(0.43 \mathrm{log}$ unit) seems unrealistic, so the case for aggregation cannot be easily dismissed.

For the aggregate model in the figure, as the $\mathrm{pH}$ is increased from 1 to 5 , the only solid present in the suspension is the free acid, $\mathrm{HA}$. As $\mathrm{pH}$ increases to 5 , the free $\left[\mathrm{Ca}^{2+}\right]$ remains constant at $1.78 \mathrm{mM}$, as $\left[\mathrm{A}^{-}\right]$ increases. When $\mathrm{pH} 5$ is reached, the salt solubility product is exceeded and the calcium salt of the drug, $\mathrm{CaA}_{2}(\mathrm{~s})$, begins to co-precipitate with $\mathrm{HA}(\mathrm{s})$ until $\mathrm{pH} 5.5$. Between $\mathrm{pH} 5.0$ and 5.5 , the calcium concentration decreases by the same extent as that of atorvastatin anion increases, but the product $\left[\mathrm{Ca}^{2+}\right]\left[\mathrm{A}^{-}\right]_{2}$ remains constant. This is the $\mathrm{pH}$ interval containing two different solids. Above $\mathrm{pH} 5.5$ (the point of maximum solubility), all $\mathrm{HA}(\mathrm{s})$ dissolves, as $\left[\mathrm{Ca}^{2+}\right]$ levels off at $0.09 \mathrm{mM}$, and only the calcium salt is predicted to precipitate, as the above product of concentrations remains constant. It may be surprising that as an added calcium salt, atorvastatin does not have a distinct Gibbs $p K_{\mathrm{a}}[42,43]$, but rather shows co-precipitation over a $0.5 \mathrm{pH}$ interval. Had the precipitate been between the drug and sodium ions, there would have been a distinct

$\mathrm{p} K_{\mathrm{a}}{ }^{\text {Gibbs }}$. The system is complex and several models can be proposed to rationalize the logS-pH profile. As can be seen, the independently-determined $\mathrm{p} K_{\mathrm{a}}$ is critical to the analysis of the data. It would have been important to isolate and characterize the solids, at least by elemental analysis, if not by comparison to PXRD of demonstrated crystalline solids of known stoichiometry. Sufficiently sensitive computations tools are needed to fully interpret such complicated systems.

\section{Amifloxacin}

Figure 1c shows the logs-pH profile of amifloxacin, an ampholyte which forms a hydrochloride salt below $\mathrm{pH} 5$ [51]. The region of maximum solubility near $\mathrm{pH} 5$ showed supersaturation effects, especially at equilibration times $<24 \mathrm{~h}$. The data in the figure are based on $48 \mathrm{~h}$ equilibration time. The unfilled circle was assigned zero weight in the refinement, since no equilibrium model could be found to fold the point into the curve represented by the other points. Also, the region between $\mathrm{pH} 5$ and 6 indicated the presence of a small 
amount of Case 2b-type in [27] cationic dimeric aggregate. This example illustrates the time dependence of the measured solubility.

\section{Ampicillin and Ampicillin Trihydrate}

Figure 1d shows the logS-pH profiles of two forms of ampicillin: anhydrous (unfilled circles) and trihydrate (filled circles) [52]. It is noteworthy that the equilibration times were $2 \mathrm{~h}$ in both cases. Had longer dissolution times been used, the more soluble anhydrous form would likely have converted into the trihydrate. Many anhydrous forms of drugs are near 2-fold higher in solubility than their hydrate counter parts $[4,18]$. The two profiles are well-described by the simple $\mathrm{HH}$ equation.

\section{Cefadroxil}

Figure 2a shows the logS-pH profile of cefadroxil, which illustrates two complicating aspect of solubility equilibria [53]. The study considered many different buffers: acetate, ammonium, borate, citrate, formate, lactate, and phosphate. The analysis of the data here excluded the measurements in citrate (unfilled squares)
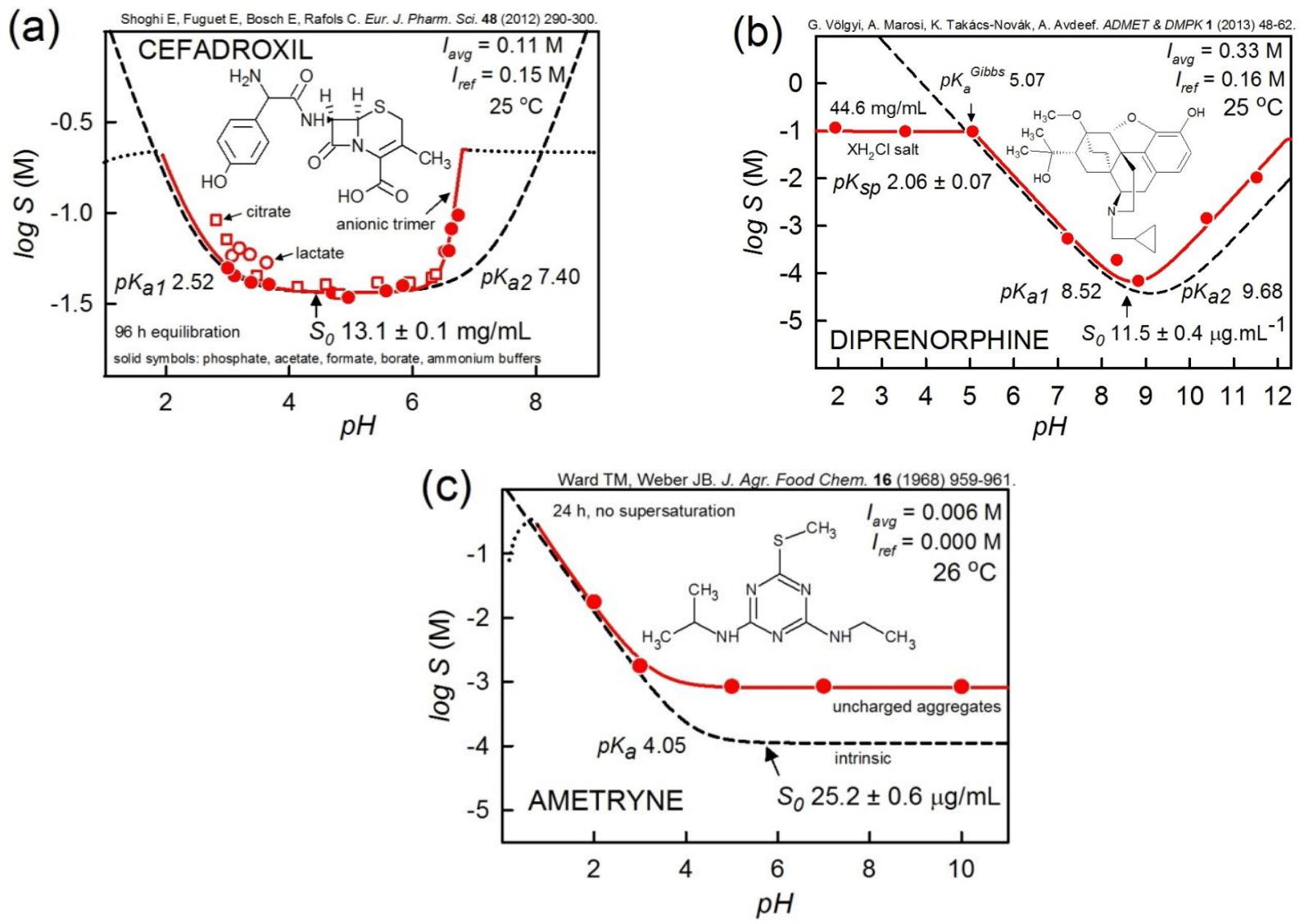

Figure 2. Shown are three examples of molecules with logS-pH profiles which are consistent with the presence of anionic or uncharged self-associated aggregates, and one case consistent with drug-buffer interaction.

and lactate (unfilled circles) buffers, since their solubility in acidic solutions tended to deviate from the curve composed of all the other buffer measurements. The elevated solubility of the lactate and citrate cases suggested that complexes might have formed between the buffer anions and the positively-charged cefadroxil, complicating the solution chemistry. The analysis of the non-complicating buffer data yielded the solid curve in the figure. Evidently, the data above $\mathrm{pH} 6$ cannot be predicted by the simple HH equation. Using $p D I S O L-X$, the 
data were best fit by assuming the presence of anionic monomers and trimers (Case 2a in [27]) of cefadroxil. Shoghi et al. [53] proceeded to analyze the solutions between pH 6 and 7 using low ionization energy ESI-QTOF-MS/MS and found direct evidence for the presence of monomers, dimers and trimers in saturated solution. This example illustrates the importance of corroborating proposed solution-phase equilibrium models by independent methods.

\section{Diprenorphine}

Figure $\mathbf{2 b}$ shows the solubility profile of diprenorphine hydrochloride, $\mathrm{XH}_{2} . \mathrm{Cl}$, in the modified Sørensen's buffer: $0.15 \mathrm{M} \mathrm{NaH}_{2} \mathrm{PO}_{4}$ adjusted with $14.85 \mathrm{M} \mathrm{H}_{3} \mathrm{PO}_{4}$ or $0.5 \mathrm{M} \mathrm{NaOH}$ [22]. The two $\mathrm{p} K$ a values of diprenorphine were carefully determined in the study by Völgyi et al. [22]. For $\mathrm{pH}>\mathrm{p} K_{\mathrm{a}}{ }^{\text {iibbs }} 5.07$, the precipitate is the uncharged ordinary ampholyte, showing the characteristic parabolic shape. At pH below the Gibbs $\mathrm{p} K \mathrm{a}$, either the chloride or the phosphate salt precipitates (or possibly both). Based on the reported solubility data, it is not definitively certain which form precipitates. More data would be needed at very low $\mathrm{pH}$. Alternatively, the salts could have been isolated and characterized. The flat shape of the curve $\mathrm{pH} 2-5$ is most consistent with chloride precipitate. A phosphate precipitate would be expected to show an upward curvature near the $\mathrm{pK}_{\mathrm{a}}{ }^{\text {Gibbs }}$. At $\mathrm{pH}>9$, the logS-pH curve shows a shift to lower $\mathrm{pH}$, compared that what would be predicted from the simple $\mathrm{HH}$ equation. The consistent interpretation of the shift is that a water-soluble mixed-charge anionic dimer forms, with the basic stoichiometry $\mathrm{XHX}^{-}$(Case $3 \mathrm{a}$ in [27]).

\section{Ametryne}

Figure 2c shows the solubility profile of ametryne [54], a weak base that appears to show free-base aggregation (Case $1 \mathrm{~b}$ in [27]). As reported, there was no indication of supersaturation effects at the $24 \mathrm{~h}$ equilibration. The $\mathrm{p} K_{\mathrm{a}}$ was spectrophotometrically determined [55]. Several other derivatives (but not all) studied by Ward and Weber [54] showed the characteristic Case $1 \mathrm{~b} \log \mathrm{S}$-pH pattern. If the free-base aggregation model were not invoked, then the refined $\mathrm{p} K_{\mathrm{a}}$ would be $3.14 \pm 0.08$, almost a log unit lower than the measured value. This example illustrates the need for independently measuring the $\mathrm{p} K_{\mathrm{a}}$, under conditions free of effects of aggregation and precipitation. Also, a mass spectrophotometric analysis of the slurry in neutral solution would have been corroboratively valuable.

\section{Haloperidol}

The salt solubility of haloperidol described by Li et al. [56, 57] raises several interesting points in assay design and data interpretation. Figure 3 illustrates the $\mathrm{pH}$-dependent formation of three crystalline salts of haloperidol: hydrochloride, mesylate, and phosphate.

In the first three frames of the figure, $\mathrm{HCl}$ or $\mathrm{NaOH}$ were used to adjust the $\mathrm{pH}$ of haloperidol, either introduced as a free base (Figure 3a) or as a hydrochloride (Figure 3b). Within the bounds of experimental errors, the results are largely the same. The data in the hydrochloride case (Figure $3 \mathbf{b}$ ) above pH 8 indicate a non-HH effect, which can be due to three situations: (i) formation of uncharged aggregates (Case 1b in [27]), or (ii) $24 \mathrm{~h}$ time not being adequate to reach full equilibration, or (iii) the formation of an oil phase (liquid-liquid phase separation, LLPS $[58,59]$ ), which is more soluble than the free-base crystalline phase (melting point, $149^{\circ} \mathrm{C}$ ). Ignoring this $[49,50]$, the logS-pH data above $\mathrm{pH} 7$ might suggest that the $\mathrm{p} K_{\mathrm{a}}$ of haloperidol at $37^{\circ} \mathrm{C}$ is 8.0. This would not be in agreement with the $\mathrm{p} K_{\mathrm{a}} 8.29$ independently determined at $37^{\circ} \mathrm{C}$ [60] and 8.60 at 
$25{ }^{\circ} \mathrm{C}$ [61]. Based solely on the solubility measurements, none of the three possibilities can be definitively ruled out, and additional investigation might be desirable.
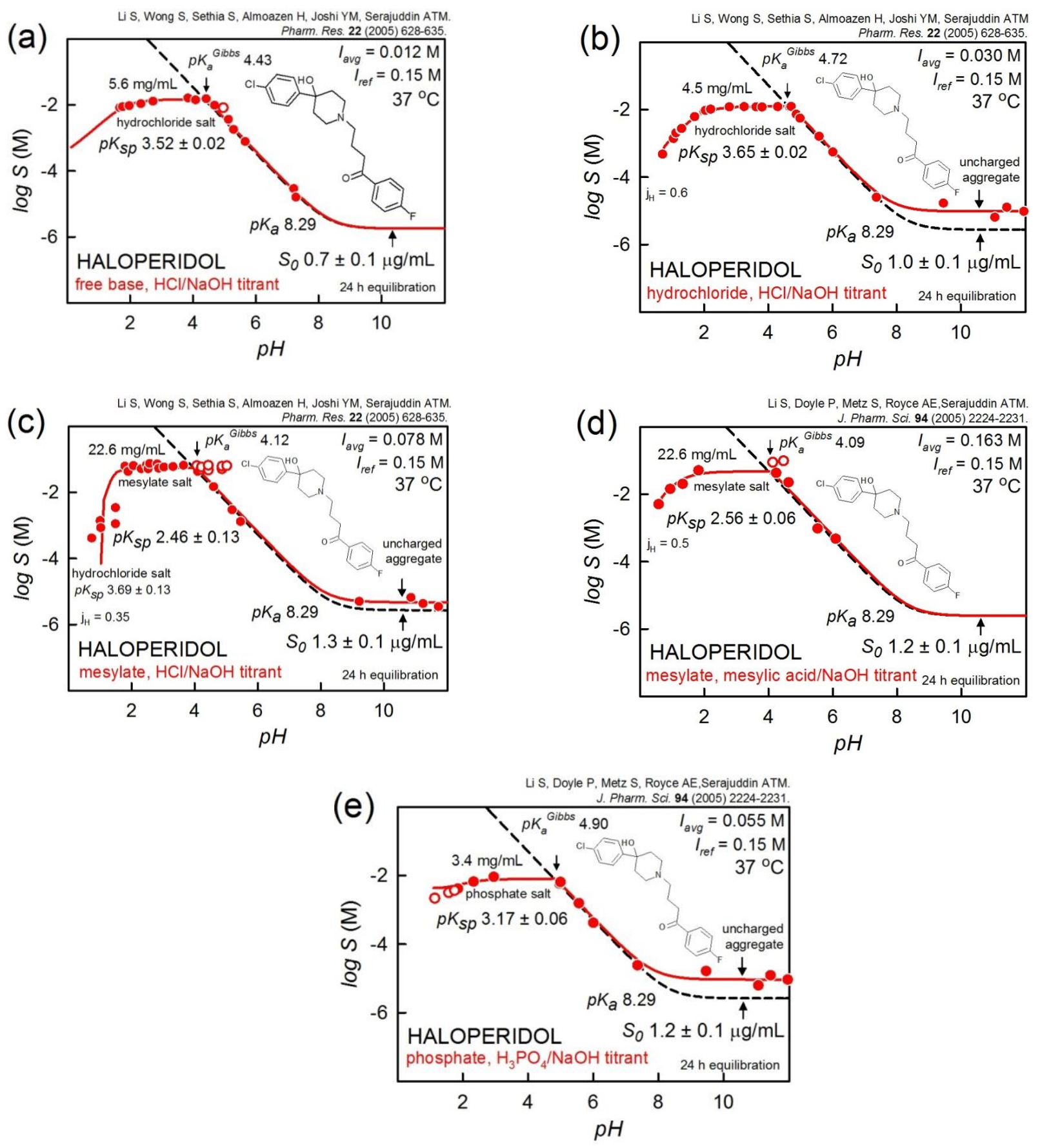

Figure 3. Examples of $\log \mathrm{S}-\mathrm{pH}$ profiles of haloperidol resulting from different salt precipitations, illustrating some degree of supersaturation near the Gibbs $p K_{\mathrm{a}}$ in the $\mathrm{pH}$ 4.1-4.9 interval and the possible formation of uncharged aggregates for $\mathrm{pH}>8$ (see text).

Figure 3c depicts the titration of the haloperidol mesylate salt by either $\mathrm{HCl}$ or $\mathrm{NaOH}$. The higher solubility in the salt precipitation region $(\mathrm{pH} \mathrm{2-4)} \mathrm{compared} \mathrm{to} \mathrm{that} \mathrm{in} \mathrm{Figures} \mathbf{3 a}$ and $\mathbf{3 b}$ suggests that the precipitate is the mesylate salt of haloperidol. However, the five lowest-pH points (Figure 3c) are not indicative of a mesylate salt. At the lowest $\mathrm{pH}$, the data were better fitted with the $K_{\mathrm{sp}}$ corresponding to the hydrochloride 
salt. Because of the very low $\mathrm{pH}(<1)$, it was necessary to invoke a $\mathrm{pH}$ electrode junction potential effect $\left(j_{H}=+0.35\right.$ [45]; cf., Appendix C). This was also needed in the cases of Figure $\mathbf{3 b}$ and $\mathbf{3 d}$, for $\mathrm{pH} \sim 1$. In the low-pH mesylate example, there is actually a region predicted to contain co-precipitates of both $\mathrm{BH} . \mathrm{Cl}(\mathrm{s})$ and BH.mesylate(s). Figure 3d is an example of haloperidol introduced as the mesylate salt and titrated with either mesylic acid or $\mathrm{NaOH}$. The same solubility is evident in the $\mathrm{pH}$ 2-4 region as that shown in Figure 3c. The precipitous drop in solubility at low pH in Figure 3c arises as a "common ion" effect, namely that in the pH 2-4 region, the concentration of chloride contributed by the $\mathrm{HCl}$ titrant is not high enough to exceed the solubility product of $\mathrm{BH} . \mathrm{Cl}(\mathrm{s})$, until enough $\mathrm{HCl}$ had been added to reach the very low $\mathrm{pH}$ values $(\mathrm{pH}<2)$.

In Figures $\mathbf{3 c}$ and $\mathbf{3 d}$, the unfilled circles were assigned zero weights in the regression analysis. The reasonable explanation for their deviancy from the models is due to supersaturation effects.

Figure $3 \mathbf{e}$ is an example of haloperidol introduced as the phosphate salt and titrated with either phosphoric acid or $\mathrm{NaOH}$. No way was found to rationalize the three points (unfilled circles) with the lowest $\mathrm{pH}$, so they were assigned zero weights.

It is interesting to note in the haloperidol case study that the order of drug salt solubility $(\mathrm{pH} 2-4)$ is: mesylate $(\sim 23 \mathrm{mg} / \mathrm{mL})>$ chloride $(\sim 5 \mathrm{mg} / \mathrm{mL})>$ phosphate $(\sim 3 \mathrm{mg} / \mathrm{mL})$. However, the order of the negative log salt solubility products is different: mesylate $(\sim 2.5)<$ phosphate $(\sim 3.2)<$ chloride $(\sim 3.5)$. This highlights the notion that salt solubility is a conditional constant, depending on the concentrations of both the drug and the counterion, whereas the salt solubility product is a true equilibrium constant.

As is evident, the interesting haloperidol case study invokes several issues where experimental design has significant consequences: titrant used to adjust $\mathrm{pH}$, electrode calibration, supersaturation, and possibly aggregation (or related phenomena).

\section{Thiazolyloxamic Acid Derivative}

Figure 4 shows a very complex solubility-pH profile of a surface-active weak acid, N-[4-(1,4-benzodioxan-6yl)-2-thiazolyl]oxamic acid, which has two $\mathrm{p} K_{\mathrm{a}} \mathrm{s}$ : 1.32 and 10.31, both determined spectrophotometrically [6]. It is an example of a molecule, when added as a salt, that becomes more soluble as more of it is added to solution, somewhat like the case of amiodarone [24]. The prominent "bump" in the logS-pH profile is consistent with the formation of a hexameric anionic aggregate, $\left(\mathrm{HA}^{-}\right)_{6}$. The solubility of the ethanolamine salt in the neutral $\mathrm{pH}$ region is $20.3 \mathrm{mg} / \mathrm{mL}\left(\mathrm{p} K_{\mathrm{sp}} 4.22\right)$. Since aggregation is strongly dependent on concentration, in the absence of aggregation (i.e., at lower concentrations), the salt solubility would be predicted to be $2.0 \mathrm{mg} / \mathrm{mL}$ (simulated blue curve in Figure 4a). Here, salt solubility depends not only on the amount of ethanolamine but also the amount of the conjugate anion, which in turn is affected by the extent of aggregation. Pandit et al. [6] also studied other salt-forming counterions, besides ethanolamine. Figure 4a shows the calcium salt solubility (dash-dot curve). The calcium salt is quite a bit less soluble than the one based on ethanolamine. For the case of calcium, the impact of aggregation is much lessened, as is suggested in Figure 4a. 

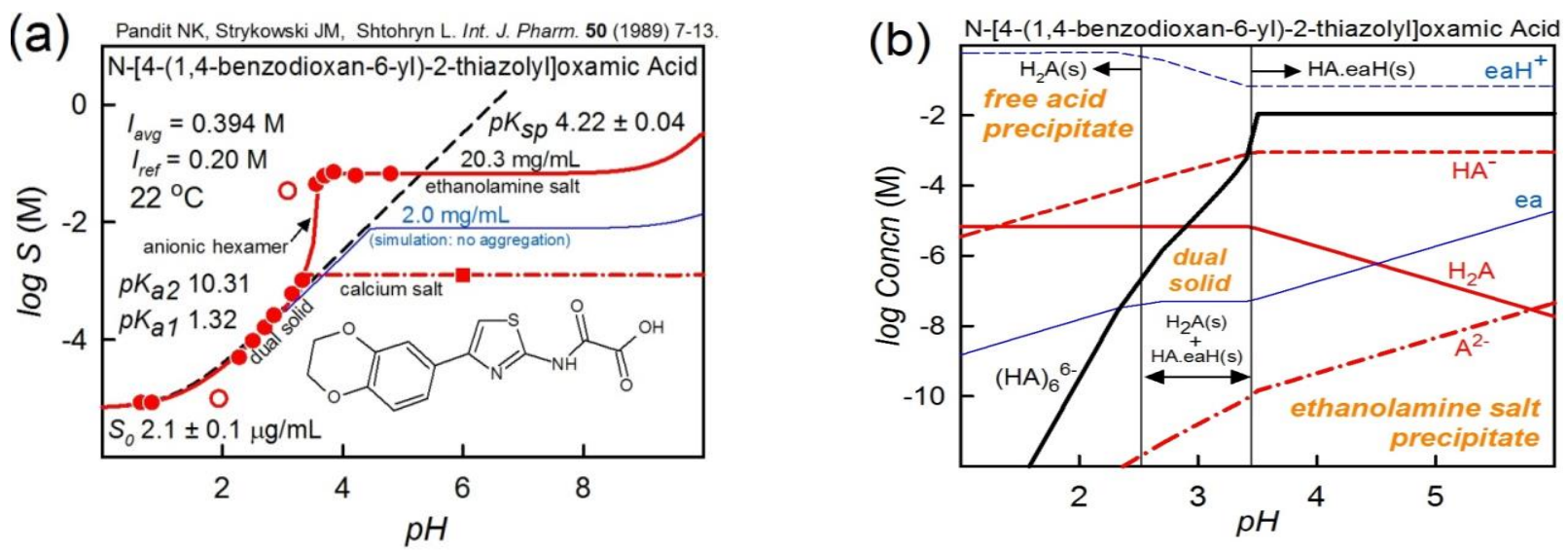

Figure 4. N-[4-(1,4-benzodioxan-6-yl)-2-thiazolyl]oxamic acid as an example of complexity of logS-pH profiles of surface-active molecules which precipitate both as salts and as uncharged species.

Another interesting feature of the oxamic acid derivative solubility profile is that between $\mathrm{pH} 2.5$ and 3.5 , the free acid, $\mathrm{H}_{2} \mathrm{~A}(\mathrm{~s})$, and the ethanolamine salt, $\left[\mathrm{HA}^{-}\right.$.(ethanolamine) $\left.\mathrm{H}^{+}\right](\mathrm{s})$, co-precipitate. Figure $4 \mathbf{b}$ identifies three solid phase regions, with boundaries delimited by thin vertical lines. Below $\mathrm{pH} 2.5$, only the free acid precipitates. Above $\mathrm{pH} 3.5$, only the ethanolamine salt of the drug precipitates. Between the two boundaries, both solids co-precipitate. A rationalization of this phenomenon is that while there is sufficient amount of the acid present to form a precipitate, there is simultaneously enough of the negatively-charged acid and the positively-charge ethanolamine to exceed the solubility product, to result in the simultaneous formation of the second solid. The presence of the hexamer (thick solid line in Figure $\mathbf{4 b}$ ) mitigates the process, since a lot of the negatively-charged acid is tied up simultaneously in the aggregate.

\section{Acetylpromazine}

Figure $\mathbf{5}$ is an example of the speciation of acetylpromazine maleate, a weak base with a reported critical micelle concentration of $12 \mathrm{mM}$ [62]. The traditional view of simple ionic detergents is that as the concentration of added surfactant increases, monomers form until the CMC is exceeded. Further increases of the surfactant lead to the formation of micelles with aggregation numbers reaching 50 or higher, whose concentration increases, while that of the monomer remains constant. With ionizable drug molecules that have surfactant properties, the picture can be more complicated, as illustrated in Figure $\mathbf{5}$. The water-soluble sub-micellar aggregate, $\left(\mathrm{B}_{9} \mathrm{H}_{7}\right)^{7+}$ best fits the "bump" portion of the logS-pH profile. The concentration of the monomer does not remain constant, and that of the aggregate formation strongly depends on $\mathrm{pH}$, as shown in Figure 5b. Ion-pair interactions between chloride and the protonated form of acetylpromazine are also suggested at low pH. There may be neutral aggregates above $\mathrm{pH} 8$ (Case $1 \mathrm{~b}$ in [27]).

For additional characteristics of the fascinating properties of acetylpromazine maleate, a close inspection of the analysis detail summarized in Figures $\mathbf{5 a}$ and $\mathbf{5 b}$, and a review of the paper by Liu and Hurwitz [62] is recommended. 

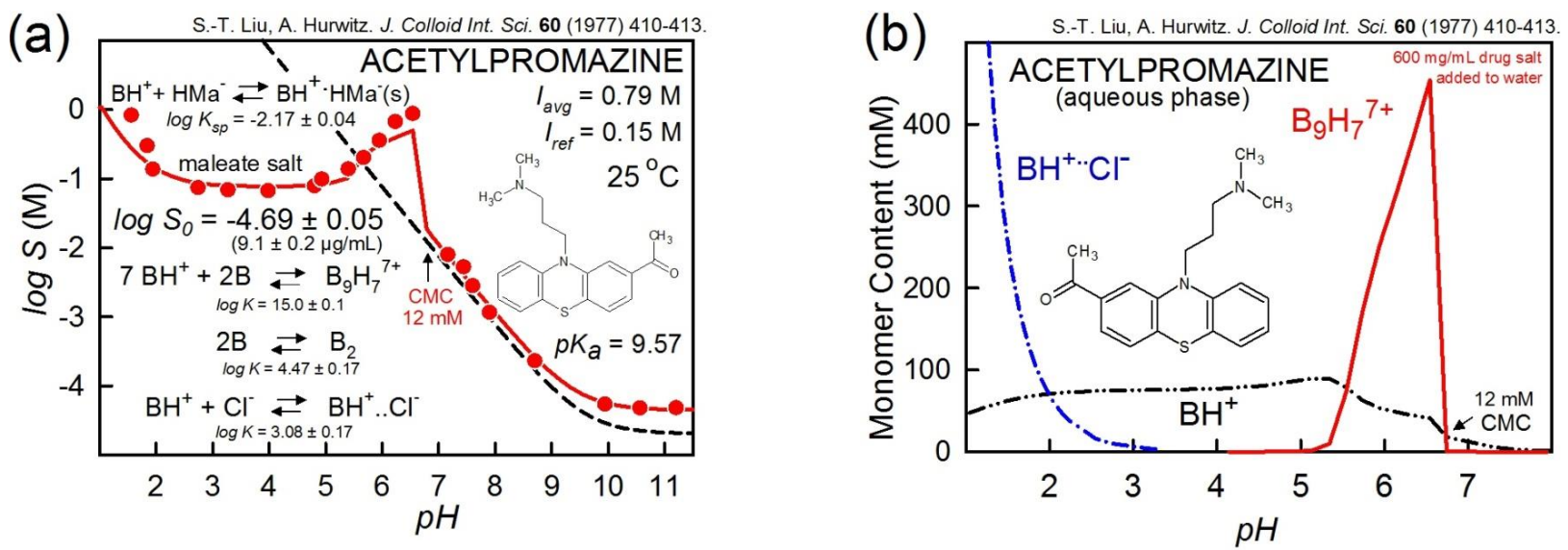

Figure 5. Acetylpromazine as an example of the complexity of $\log S$-pH profiles of surface-active molecules, suggesting that care needs to be exercised in interpreting the shapes of the solubility-pH profiles.

\section{Summary of Issues Raised in the Case Studies}

The above cases were selected as representative examples of apparently well-designed assays, which illustrate how complicated heterogeneous chemical reactions involving druglike molecules can get. There was much to consider. In several instances, it was important to know the true $\mathrm{p} K_{\mathrm{a}}$ prior to assessment of solubility$\mathrm{pH}$. When ionic strength was excessive, or when very low $\mathrm{pH}$ was considered, the calibration of the $\mathrm{pH}$ electrode required special considerations. Supersaturation had to be recognized and accordingly handled. Distortions of the logS-pH curves due to aggregation effects had to be recognized and appropriately interpreted. There were surprises regarding the $\mathrm{pH}$ range over which co-precipitates (free-acid/base drug plus drug salt) could form, parting from the single-pH significance of the " $\mathrm{pH}_{\max }$ " or "Gibbs $\mathrm{p} K_{\mathrm{a}}$ " ideas [42, 43]. It needs to be kept in mind that hydrates can form over long equilibration times. Mass spectrometry could be used to confirm the aggregate models derived from the computational analysis of logS-pH data. The identity of the salt forms of drug precipitates might not be obvious unless the assays were critically designed. More than one salt may form over a $\mathrm{pH}$ range, and sometimes the salts can overlap over a significant range of $\mathrm{pH}$. Several examples of water-soluble drug-buffer complexes were suggested. In systematic testing of salts of a given drug, the salt solubility order may not be the same as the $K_{\mathrm{sp}}$ order. The interpretation of the CMC is more complicated with ionizable drugs than often recognized.

Most of the follow-up suggestions that can be made to some of the above cases could be to pursue additional corroborative investigations to confirm the presence of aggregates, to identify the stoichiometries of the solids precipitating, and the like. Unusual stoichiometry results are seldom confirmed in a different laboratory, so reliability of the models proposed can be difficult to assess. Universally, it seems that not enough attention had been devoted to the performance of the $\mathrm{pH}$ electrodes, particularly at very low $\mathrm{pH}$. A definitive discussion of what is meant by "supersaturation" at the molecular level has not been published, as far as we are aware. It's a term that is used frequently and has an intuitive appeal but the exact molecular mechanism has not been described. Do transient micelles and/or sub-micellar aggregates, or drug-excipient complexes, form in a supersaturated solution? Does meta-stable particle formation, or high energy interactions caused by energetic phase contacts, or increased surface tension lead to the supersaturation?

Examples of poorly designed solubility assays were not considered as case studies. Such assays outnumber 
the well-designed measurements by a landslide. The goal of this commentary is to present specific recommendations that could elevate the quality of assay design, to lead to improved quality of measurements, and impart a deeper understanding of the underlying solution chemistry in the presence of drug precipitates, and how such solids can influence the interpretation of the solubility measurement.

\section{Methods for Measuring Solubility}

\section{"Gold Standard" Shake-Flask Solubility Method}

The traditional saturation shake-flask (SF) method is based on simple, easy-to perform procedures, but it is time-consuming and labor intensive. Without expensive instrumentation, SF can be conducted in any standard analytical laboratory. But to get precise and accurate solubility results, several critical experimental conditions have to be considered. When the SF method is properly performed, according to a well-designed protocol, it provides high quality data with standard deviation lower than $\pm 5 \%$.

The SF method starts with the preparation of a sample suspension, the solution of the tested compound in the selected solvent (mainly in aqueous buffer - see the section below) containing a small excess of the solid. (A large excess in the $\mathrm{pH}$ region where the compound converts from salt form to a free acid/base should be avoided, since the disproportionation may result in the free acid/base coating the surface of undissolved drug salt, which could result in confusing characterization of the isolated solid state material.) The volume of the suspension that should be used depends on the solubility of the sample and the concentration detection method. For majority of compounds, when the saturated solution has to be diluted for assay, 1-3 mg in $3 \mathrm{~mL}$ in small $(10 \mathrm{~mL}$ ) glass vial can be optimal for precise work. In such volume the $\mathrm{pH}$ control is easy and one can easily follow visually any changes in the vial during the equilibration. For extremely insoluble compounds, $(\mathrm{S}<10 \mu \mathrm{g} / \mathrm{mL}$ ) the concentration has to be measured (when using UV spectrophotometry) without dilution; thus bigger volume of solubility suspension ( $1 \mathrm{mg}$ solid in $15-20 \mathrm{~mL}$ solvent) is needed for three replicates of sampling or when $5 \mathrm{~cm}$ pathlength UV cell has to be used. It is a good practice to measure the $\mathrm{pH}$ of the solubility suspension $1 \mathrm{~h}$ after preparation and readjust the desired $\mathrm{pH}$ value if it is shifted which frequently happens when compound is applied in salt form (Table 1).

The heterogeneous system is capped and vigorously agitated (stirred) at a chosen temperature $\left(25,37^{\circ} \mathrm{C}\right.$ or other) in a thermostated bath for a specified time $(6,24,48 \mathrm{~h}$ or longer - see the section below) until the solubility equilibrium has been reached. After that, the solid is separated from the solution by sedimentation, centrifugation or filtration (see below). Upon diluting the sample aliquots with the solvent, if necessary, the concentration of the saturated solution is measured by an appropriate method, most frequently by UV-Vis or HPLC/UV-Vis. The final pH of the saturated solution is measured.

Due to the lack of generally accepted standard way to carry out of this method, the published solubility studies show great differences in the experimental conditions used [4]. Baka et al. [3] suggested a new protocol for SF method using small excess of solid, $6 \mathrm{~h}$ stirring and $18 \mathrm{~h}$ sedimentation time and running a minimum of 3 replicates. This protocol was applied for close to 100 compounds of different structures, acidbase property, morphology, etc., and found appropriate for the precise measurement of equilibrium solubility [63]. 
Table 1. $\mathrm{pH}$ Shift of solubility suspensions upon preparation

\begin{tabular}{ccccc}
\hline Sample & $\begin{array}{c}\text { pH of BR } \\
\text { buffer }^{\mathbf{a}}\end{array}$ & $\begin{array}{c}\mathbf{p H} \text { of BR buffer } \\
\text { after addition } \\
\text { of solid }(\boldsymbol{t}=\mathbf{1 ~ h} \mathbf{~})\end{array}$ & $\begin{array}{c}\text { Titrant added to } \\
\text { readjust the } \mathbf{p H}\end{array}$ & $\begin{array}{c}\text { Final pH of the } \\
\text { saturated } \\
\text { solution }(\boldsymbol{t}=\mathbf{2 4} \mathbf{h})\end{array}$ \\
\hline $\begin{array}{c}\text { Desvenlafaxine } \\
\text { fumarate }\end{array}$ & 9.5 & 5.0 & $\mathrm{NaOH}$ & 9.5 \\
$\begin{array}{c}\text { Promethazine } \\
\text { hydrochloride }\end{array}$ & 11.0 & 6.6 & $\mathrm{NaOH}$ & 10.8 \\
$\begin{array}{l}\text { Quetiapine fumarate } \\
\text { Rosuvastatin }\end{array}$ & 3.0 & 4.3 & $\mathrm{H}_{3} \mathrm{PO}_{4}$ & 3.0 \\
\hline
\end{tabular}

${ }^{\mathrm{a}} \mathrm{BR}=$ Britton-Robinson buffer (cf., Appendix A).

\section{CheqSol Intrinsic Solubility Method}

An alternative to the shake-flask approach for measuring the intrinsic solubility of ionizable compounds is the potentiometric acid-base titration method introduced by Avdeef [42]. It is not necessary to separate the solid from the solution in the potentiometric approach (cf., Separating Solid from Saturated Solution). The intrinsic solubility is calculated from the shift between the apparent $\mathrm{p} K_{\mathrm{a}}$ (in the presence of precipitate) and the true aqueous $\mathrm{p} K_{\mathrm{a}}$ (in the absence of solid). In order to measure the true intrinsic solubility, equilibrium must be reached. This can take quite a long time, especially near the regions of complete dissolution. In order to ensure equilibrium is actually reached and in a short time, a method called CheqSol (abbreviation of "Chasing equilibrium Solubility") was introduced by Stuart and Box [64], and subsequently developed by Sirius Analytical (UK). The method has been validated [65] and comparison of the intrinsic solubility results measured by the SF and CheqSol techniques, when both are properly performed, shows good agreement with a weighted linear regression of $\log S_{0}{ }^{S F}=-0.13+1.00 \log S_{0}{ }^{\text {CheqSol }}\left(r^{2}=0.90, s=0.52, n=125\right)$ [4].

The method is based on the shift of the Bjerrum's plot (cf., Glossary) for the titration when precipitate is present (Figure 6). The method consists of dissolving the material in water and seeking precipitation by backtitrating the resulting solution (adding measured aliquots of base or acid titrant) until first precipitation is detected. After initial precipitation of the neutral species has taken place, the solution is switched from supersaturated to subsaturated solutions and back again several times, seeking an equilibrium $\mathrm{pH}$ where the sample is neither further precipitating nor re-dissolving.

The method quickly pinpoints equilibrium, by advancing the $\mathrm{pH}$ further in the direction that it was already changing spontaneously. In contrast, the Dissolution Titration Template (DTT) potentiometric method passively allows equilibration to be reached [42]. When precipitation is occurring, the $\mathrm{pH}$ will change in one direction; when dissolution is occurring, $\mathrm{pH}$ will change in the opposite direction (see Figure 7). Therefore, by following the $\mathrm{pH}$ gradient, the appropriate titrant can be added to accelerate $\mathrm{pH}$ change in the direction of equilibration, above its ordinarily slow change. Inevitably, such titrant additions overshoot the equilibrium point, at which point the gradient will be reversed. A smaller amount of the counter titrant is then added. A change in $\mathrm{pH}$ of less than 0.05 is usually sufficient to reverse the direction of the $\mathrm{pH}$-gradient. Such active $\mathrm{pH}$ 
nudging, termed "chasing equilibrium," is done as many times as necessary. The interpolated zero points of the $\mathrm{pH}$-gradient indicate that the system is at equilibrium, when neither precipitation nor dissolution is observed. Each cycle between a supersaturated solution and a subsaturated one will produce a measure of the

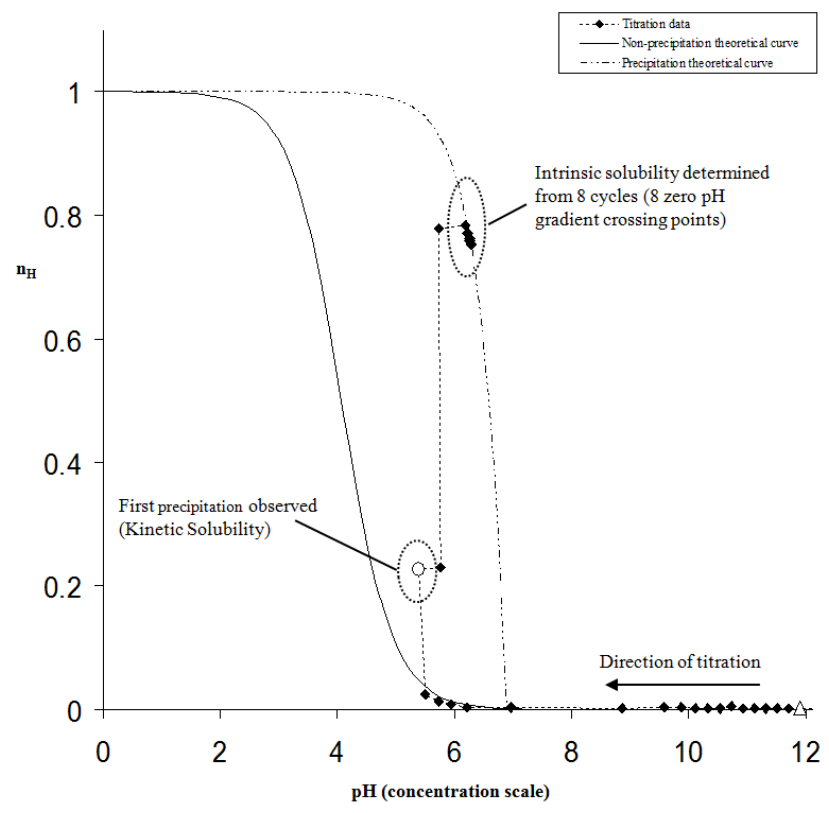

Figure 6. Bjerrum curve (average number of associated protons $\left(n_{\mathrm{H}}\right) \mathrm{vs} \mathrm{pH}$ ) for diclofenac. The experimental data follows the theoretical curve up to the precipitation point (full circle), when it jumps onto the precipitation curve. The direction of titration is towards the acidic region (right to left).

intrinsic solubility (Figure 7b). Usually the apparent intrinsic solubility values are distributed in a tight group around the average (true) value. If the intrinsic solubility values are not randomly distributed (like in Figure 7a), and show a systematic drift in either direction, then this means that the equilibrium has not been reached (or that a more stable polymorph can be generated), and the number of cycles is increased until the equilibrium condition is achieved. In the case of shake-flask measurements the system is allowed to shake for 6-48 $\mathrm{h}$ followed by long sedimentation times (cf., "Gold Standard" Shake-Flask Solubility Method), in an attempt to reach equilibrium, which is then assumed. In the CheqSol case it is easy to see if the equilibrium has been reached, and it is usually achieved for most compounds in 1-2 $\mathrm{h}$ (8 cycles).

Although not adapted to use auxiliary buffers, the CheqSol technique applies mass balance equations to rigorous nonlinear least squares refinement of the model based on the Henderson-Hasselbalch equation. However, as described previously in this manuscript, a very accurate determination of the $\mathrm{p} K_{\mathrm{a}}$ of the sample is therefore needed, because the ionization constants will affect the accuracy of the concentration of the neutral species, using the Henderson-Hasselbalch relationship. Therefore a very accurate $\mathrm{p} K_{\mathrm{a}}$ needs to be measured, preferably at exactly the same conditions (temperature, ionic strength, ...).

It is not recommended to use a "predicted" value or a $\mathrm{p} K_{\mathrm{a}}$ derived from the logS-pH curve (since the Henderson-Hasselbalch equation, in many cases, may not be valid). The spread of the crossing points in CheqSol is used to determine the coefficient of variation (CV) of the mean intrinsic solubility result, which is usually $<4 \%$. The final intrinsic solubility value is calculated from an average of several separate experiments with $\sim 8$ cycles per experiment, adequate to provide the intrinsic solubility value with an associated statistical reproducibility error $( \pm S D)[1]$. 

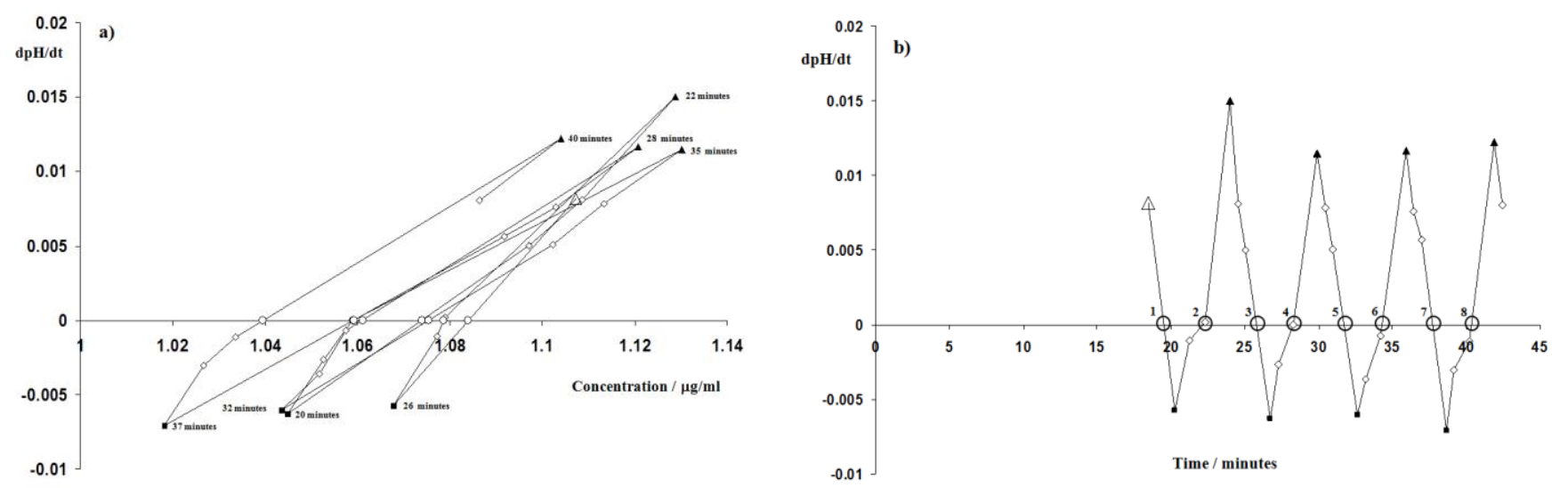

Figure 7. Chasing equilibrium of diclofenac ( 1 of 10 runs): the $\mathrm{pH}$ gradient $(\mathrm{dpH} / \mathrm{d} t)$ changes from a positive value (neutral form is precipitating) in the supersaturated region to a negative one (neutral form is re-dissolving) in the subsaturated one: the rate of $\mathrm{pH}$ change is zero at the crossing points (0). In this run eight cycles were performed ( 8 crossing points). Each crossing point in plot a) corresponds to an intrinsic solubility value in b). That is, the first crossing point, point 1 in plot a) occurs at around 20 min after the experiment has started, giving an intrinsic solubility value (first crossing point) of $1.06 \mu \mathrm{g} / \mathrm{ml}$ (plot b); point 2, $22 \mathrm{~min}$ and $1.07 \mu \mathrm{g} / \mathrm{ml}$; point 3, $26 \mathrm{~min}, 1.08 \mu \mathrm{g} / \mathrm{ml}$; point 4, 28 $\min , 1.07 \mu \mathrm{g} / \mathrm{ml}$; point 5, $32 \mathrm{~min}, 1.06 \mu \mathrm{g} / \mathrm{ml}$; point 6, $35 \mathrm{~min}, 1.07 \mu \mathrm{g} / \mathrm{ml}$; point 7, $37 \mathrm{~min}, 1.06 \mu \mathrm{g} / \mathrm{ml}$ and point 8, 40 $\min , 1.04 \mu \mathrm{g} / \mathrm{ml}$. Full triangles $(\boldsymbol{\Delta})$ represent when acid titrant is added; squares ( $\boldsymbol{\square})$ when base titrant is added, $(\Delta)$ mean no titrant added; (O) are the crossing points, when the $\mathrm{pH}$ does not change, and the large empty triangle is where the experiment starts.

The measurements are performed under inert gas (argon or nitrogen) and degassed reagents and water are used (minimal dissolved $\mathrm{CO}_{2}$ ). The total ionic strength, $l$, is kept nearly constant at $0.15 \mathrm{M} \mathrm{KCl}$ and the temperature is precisely controlled throughout (e.g., $25.0 \pm 0.1^{\circ} \mathrm{C}$ ). The $\mathrm{pH}$ electrode is calibrated using the "Four-Plus" method (cf., Appendix C).

Diclofenac is shown below as an example [66]. Figure 6 shows the Bjerrum curve for diclofenac. For fully dissolved diclofenac the titration curve is calculated from the $\mathrm{p} K_{\mathrm{a}}$ of diclofenac (measured previously at the same conditions as the solubility experiments) and corresponds to the continuous line in Figure 6 . Aqueous pH titration was not used to determine the $\mathrm{p} K_{\mathrm{a}}$ because of precipitation problems. Instead, several titrations were performed at different concentrations of co-solvent (methanol) and the aqueous $\mathrm{p} K_{\mathrm{a}}$ was obtained by extrapolation to zero methanol concentration (Yasuda-Shedlovsky method) [27, 67-69]. The average $\mathrm{p} K_{\mathrm{a}}$ of $4.08 \pm 0.04$ was obtained $\left(n=10, I_{\text {ref }}=0.15 \mathrm{M}, 25^{\circ} \mathrm{C}\right)$, which agrees closely with previous reported value of 3.99.

In the CheqSol method, diclofenac sodium is titrated with standardized $\mathrm{HCl}$ (Figure 6, high-to-low $\mathrm{pH}$ direction), until precipitate is turbidimetrically detected (filled circles). At this point the measurements depart suddenly from the calculated Bjerrum curve, because of the change in the amount of dissolved diclofenac. The solution is then repeatedly switched (by $\mathrm{HCl} / \mathrm{KOH}$ titrant additions) from subsaturated to supersaturated states (Figure 7), until the value for the transition between the states, the solubility estimate, has converged. The intrinsic solubility is calculated as the mean concentration of all the interpolated crossing points. The Bjerrum curve for the saturated solution is illustrated as the dot-dashed line in Figure 6. The experiment was repeated ten times and a new intrinsic solubility value is calculated as the mean of all ten converged intrinsic solubility values. 
Results for one solubility determination of the sodium salt of diclofenac are illustrated in Figure 7, which represents the $\mathrm{pH}$ gradient against the concentration of the neutral species. Each crossing point represents the transition from supersaturated to subsaturated (or vice-versa) and so the concentration of the neutral species at zero $\mathrm{pH}$ gradient is the intrinsic solubility $\left(S_{0}\right)$ of diclofenac. All crossing points should lie close together and give essentially the same answer. The agreement in Figure $\mathbf{7}$ is excellent, with a coefficient of variation (CV) of $1.2 \%$. The spread of these crossing points is used to determine the CV of the mean intrinsic solubility result, and as pointed out earlier, to check if equilibrium has been reached. If the crossing points lie closely together (low CV) this means that the sample is poised at equilibrium, but if the crossing points are not in a tight bunch (high CV) or they are not randomly distributed (showing a tendency towards one single intrinsic solubility value) that means that the system needs more time to evolve towards steady state.

The diclofenac free acid was isolated by stopping the solubility experiment after eight cycles at $\mathrm{pH}$ 6.5. At this $\mathrm{pH}$ and at the end of the experiment ( $60 \mathrm{~min})$, the solid precipitate is in equilibrium with the neutral form in solution. Differential scanning calorimetry, thermogravimetric analysis and powder X-ray diffraction were performed on this precipitate in order to fully characterize the solid. The crystal structure of this solid corresponded to the structure SIKLIHO1 (Cambridge Structural Database) [70]. Characterization showed that the precipitated solid is the anhydrous form of the acid of diclofenac, space group $\mathrm{C} 2 / \mathrm{c}$, with an observed melting point of $180.5^{\circ} \mathrm{C}$.

\section{Potentiometric Cycling for Polymorph Creation, $[P C]^{2}$}

Any solubility measurement depends on the form of the solid in equilibrium with its saturated solution. It is therefore not enough to know the solid form of the material at the beginning of the experiment. Using the CheqSol method one avoids the possibility of confounding different forms precipitating, and therefore reporting misleading data (i.e., amorphous vs crystalline, salts vs neutral forms, hydrates vs anhydrous...). Pudipeddi and Serajuddin published a survey in 2005, showing that the difference in solubility of polymorphs is usually less than a factor of two [18]. However our results suggest that polymorphs [71] can have a considerably greater factor than this. It is therefore of vital importance to properly characterize the solid form, the solubility of which is reported, but this is rarely done in the literature.

CheqSol can be used to parse the entire Meta Stable Zone (MSZ) shown in Figure 8, starting from the first form precipitating at high supersaturation, which is the highest energy polymorph (kinetically driven), down to the last most stable form on the thermodynamic solubility saturation curve (thermodynamically driven) and achieved by cycling the system many times between supersaturation and subsaturation. This transition is normally seen as a continuous transition from the first precipitate to the most stable form, following Ostwald's rule (see Figure 8). Metastable polymorphs encountered during this path are not, in general, stable enough to isolate and characterize, but sometimes a metastable polymorph arises during this process, being stable enough to reach a $100 \%$ of this metastable polymorph, which then can be isolated and characterized. Nevertheless, if one continues cycling between supersaturated solution and subsaturated solution, this metastable form will evolve towards the most stable one, which will not change in form under the cycling process. It's an advantage that these polymorph transformations can be followed in real time, when the solubility can be measured for each different form, by stopping the cycling at a specific $\mathrm{pH}$, collecting the solid and characterizing it by several techniques. This cycling method has been called "Potentiometric Cycling for Polymorph Creation" [PC] $]^{2}$. 


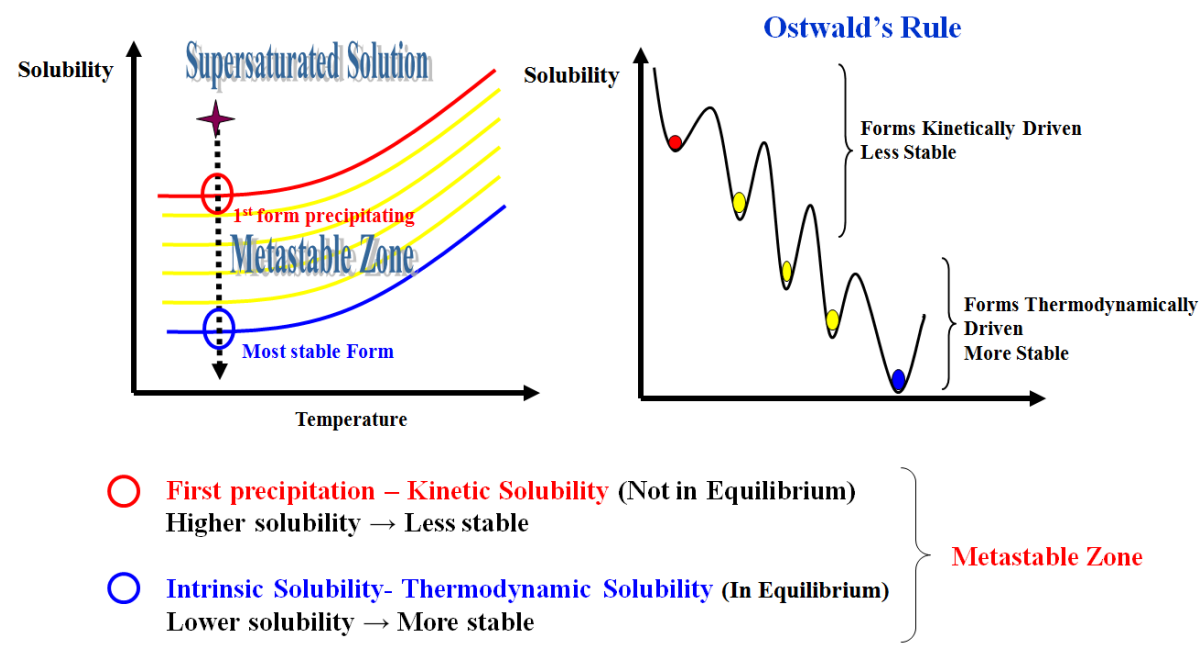

Figure 8. Supersaturation and Oswald ripening.

This transformation can also be followed in real time using a Raman probe coupled to the potentiometric instrument enabling one to follow these phase transitions without the need of stopping the experiment and isolating the solid. This technique allows one to have a precise control of the rate of transformation of one form into the other, depending on the conditions of the experiment used.
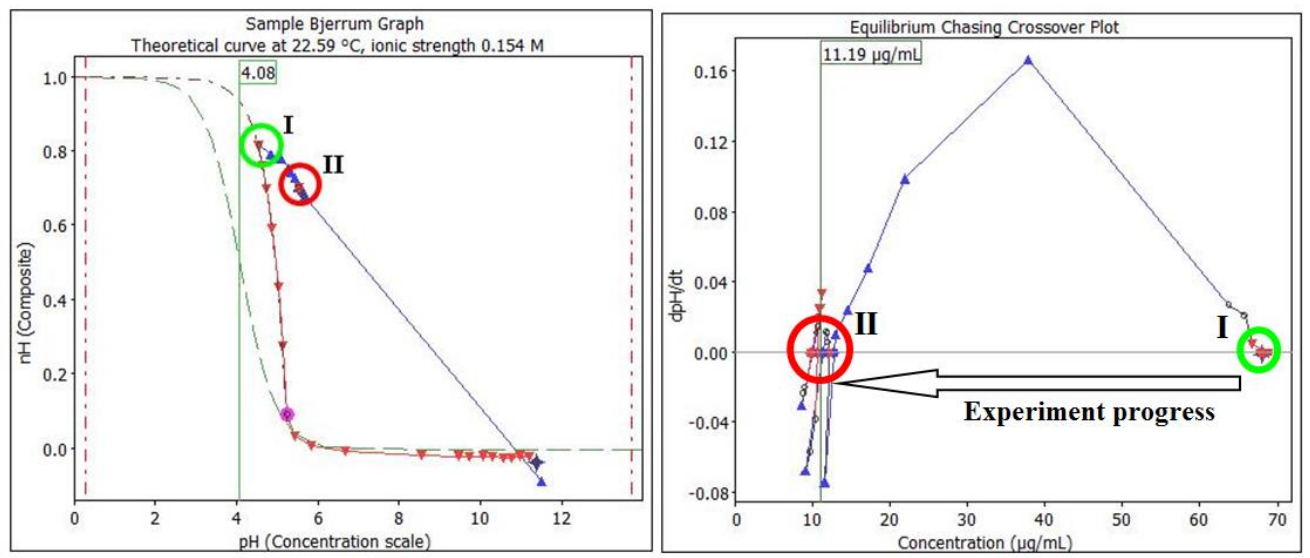

Figure 9. Bjerrum curve for sulindac depicting the first (Form I) and equilibrium cycling (Form II). Figure on the right shows the evolution of the $\mathrm{pH}$ gradient as the experiment progress. Note how the first precipitate form I has a solubility around $70 \mu \mathrm{g} / \mathrm{mL}$, while the most stable form, Form II, stablishes the cycling on a value around 10

$$
\mu \mathrm{g} / \mathrm{mL} \text {. }
$$

This approach has been applied to numerous compounds (bases, acids and zwitterions), generally obtaining the more stable polymorph; in many cases they proved to be newly-identified polymorphs $[1,66,71-74]$.

Sulindac exemplifies the [PC] ${ }^{2}$ approach [73]. At first the experiment produced measurements following the usual pattern of a CheqSol experiment and the readings converged on an intrinsic solubility of $70 \mu \mathrm{g} / \mathrm{mL}$ (Figure 9). The crystalline precipitate (Form I in Figure 9) was then isolated and characterized by powder X-ray diffraction (PXRD), Raman spectroscopy, infrared spectroscopy (IR), thermogravimetry analysis (TGA) and 
differential scanning calorimetry (DSC). The powder pattern for this form (Form I) matched the pattern reported in the Cambridge Structural Database (CSD reference code DOHREX) [75]. However, it was observed that a sudden drop in solubility from about 70 to $10 \mu \mathrm{g} / \mathrm{mL}$ occurred after cycling for $20 \mathrm{~min}$ (Figures 9 and 10).

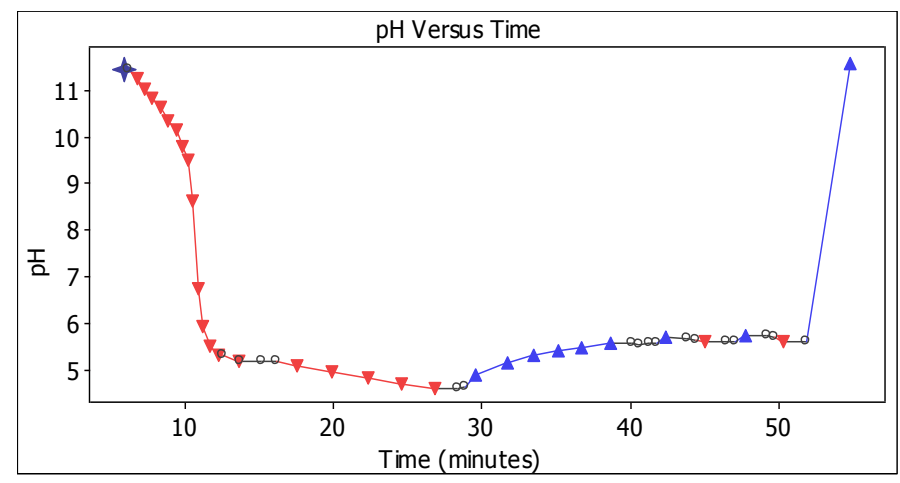

Figure 10. Evolution of $\mathrm{pH}$ vs time for the sulindac experiment. Form I is obtained in pure form if the cycling is stopped before 20 minutes (precipitation takes place at a $\mathrm{pH}=4.5$ ). Pure Form II is obtained when the cycling is kept for longer times $(40-50 \mathrm{~min}, \mathrm{pH}=5.7)$

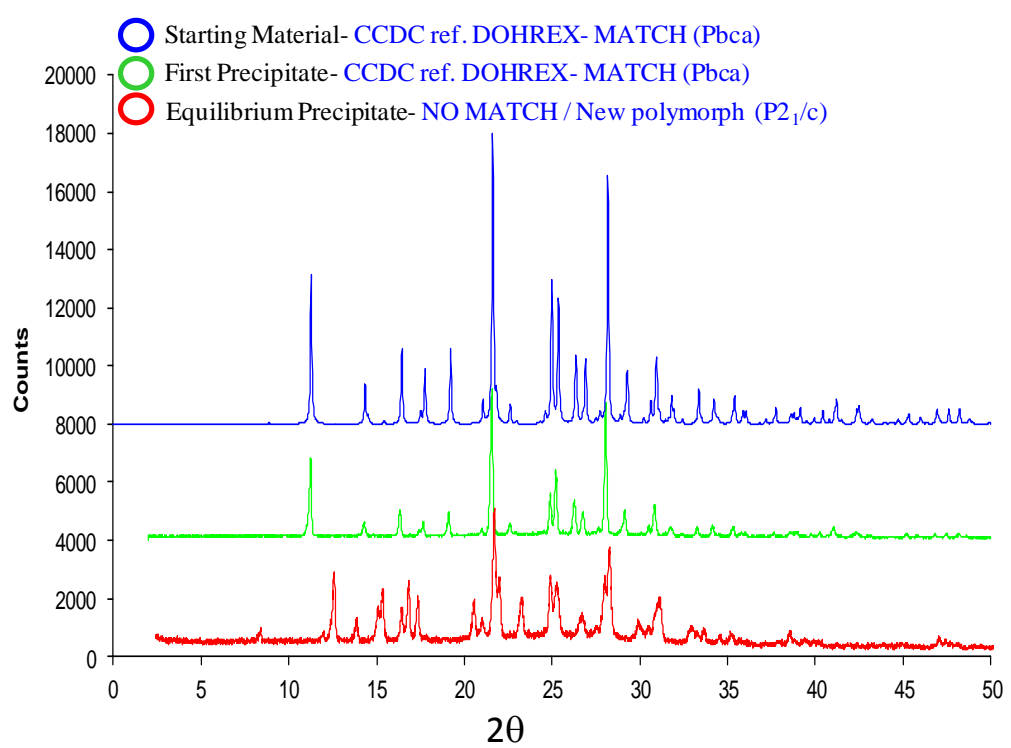

Figure 11 Powder X-ray diffraction of the different solid forms obtained during the sulindac $[\mathrm{PC}]^{2}$ experiment. The first precipitate detected in the experiment is Form I which corresponds to the orthorhombic sulindac $\mathrm{Pbca}$ already described in the CCDC database (DOHREX). Form II did not match any previously reported pattern. The new polymorph of sulindac, form II, is the most stable polymorph and corresponds to the monoclinic P21/c form. This new form has now the reference name DOHREX01 in the CCDC database.

This behavior was repeatable and setting up the conditions to cycle for more than 20 min always produced the more stable Form II with an intrinsic solubility of $10 \mu \mathrm{g} / \mathrm{mL}$. This low solubility form was isolated and characterized in the same manner as Form I but in this case the powder pattern did not correspond to anything in the CSD (Figure 11). The DSC and TGA measurements confirmed that Form II has no solvent in the crystal structure, and therefore it was not simply a different hydrate of sulindac. The crystal structure of this new form (II) was solved from the powder X-ray diffraction pattern using the simulated annealing algorithm implemented in DASH [76], and refined using the Rietveld method implemented in the General Structure Analysis System [77]. The factor of seven fold between the intrinsic solubility of the original form I of sulindac 
and this new more stable Form II is unusually large, but based on many other [PC] $]^{2}$ experiments performed on different materials, a 10 fold difference is quite common. The $[P C]^{2}$ approach is therefore a very promising technique to measure accurately and reproducibly the intrinsic solubility of different polymorphs, since the approach is able to generate metastable and stable polymorphs. Experiment to produce the hydrate or the anhydrous form exclusively (when possible) can be designed. The application of the [PC] ${ }^{2}$ technique to polymorphic systems apparently generates the most stable form of the compound, which is especially appealing to the pharmaceutical industry. By controlling the experimental conditions, also metastable forms can be obtained on purpose.

\section{Solid State Characterization, Polymorphs, Hydrates, Cocrystals}

Analysis not only of the solution, but also of the solid residue, at least at the beginning and at the end of a dissolution experiment, is often claimed to be too time-consuming, to be recommended for common use. However, the interpretation of the results of a dissolution measurement can be misleading, if solubility refers to the wrong (or unknown) solid phase. Modern powder X-ray diffraction (PXRD) techniques make it possible to follow the composition of a solid phase directly in solution in situ, and also in high-throughput screening experiments (e.g., using instruments from Panalytical [78], or Bruker [79]). When characterizing a solid sample by PXRD, it is important not to limit the analysis by considering one or two characteristic peaks, but to analyze the whole diffraction pattern. Diffraction patterns of some polymorphs can be very similar at low values of $2 \theta$ diffraction angles, so that they can be distinguished only if data are collected also at higher angles (see as an example diffraction patterns of the $\alpha, \varepsilon, \varepsilon^{\prime}$-polymorphs of chlorpropamide, Figure 12 [80]).

One can also use confocal Raman spectroscopy for fast analysis of the solid phase [81, 82], although Raman spectroscopy may be less sensitive for distinguishing between the polymorphs with common structural motifs than PXRD is. It is also very instructive to monitor a crystal of a selected original phase directly in solution using an optical microscope: quite often a recrystallization into another solid phase can be visually evident (see Figure 13 as an example), but that should never be taken as proof of different polymorphs since a single form can show multiple morphologies.

The following example further illustrates some of the kinds of unexpected results that may arise following solid-state characterization, which might surprise some practitioners. The differential scanning calorimetry (DSC) of a hydrochloride salt of an insoluble base $\left(\mathrm{p} K_{\mathrm{a}} \sim 9\right)$ indicated a sharp negative peak, corresponding to the melting point of the drug salt. The salt was added in substantial excess to a neutral solution (where not the entire solid dissolved), whose $\mathrm{pH}$ was subsequently adjusted to 10 . After $24 \mathrm{~h}$, the solid was filtered out, rinsed and dried, and had its DSC run. A much lower melting point corresponding to the free base was expected.

However, the melting point actually measured by DSC was identical to the original drug salt starting material. A possible explanation is as follows. When a practically insoluble base (e.g., thioridazine) is added as a hydrochloride salt in large excess to a neutral solution, and the resultant suspension is then quickly made alkaline $\left(\mathrm{pH}>\mathrm{p} K_{\mathrm{a}}\right)$, it is possible that the drug released from the dissolving salt particles immediately reprecipitates as the free base on the surface of the salt particles, quickly encasing them. These free-base coated particles of undissolved salt can be stable in solution. The suspension may reach a steady state after $24 \mathrm{~h}$, regulated by the surface coating (which may be amorphous, or crystalline, or oil) of the practically-insoluble free base, although the suspension contains considerable undissolved crystalline salt, insulated from the 
aqueous solution. The filtered solid can show a melting point corresponding to crystalline drug salt, rather than the expected free base. This example illustrates the value of solid-state characterization and the need to critically examine assay protocols.

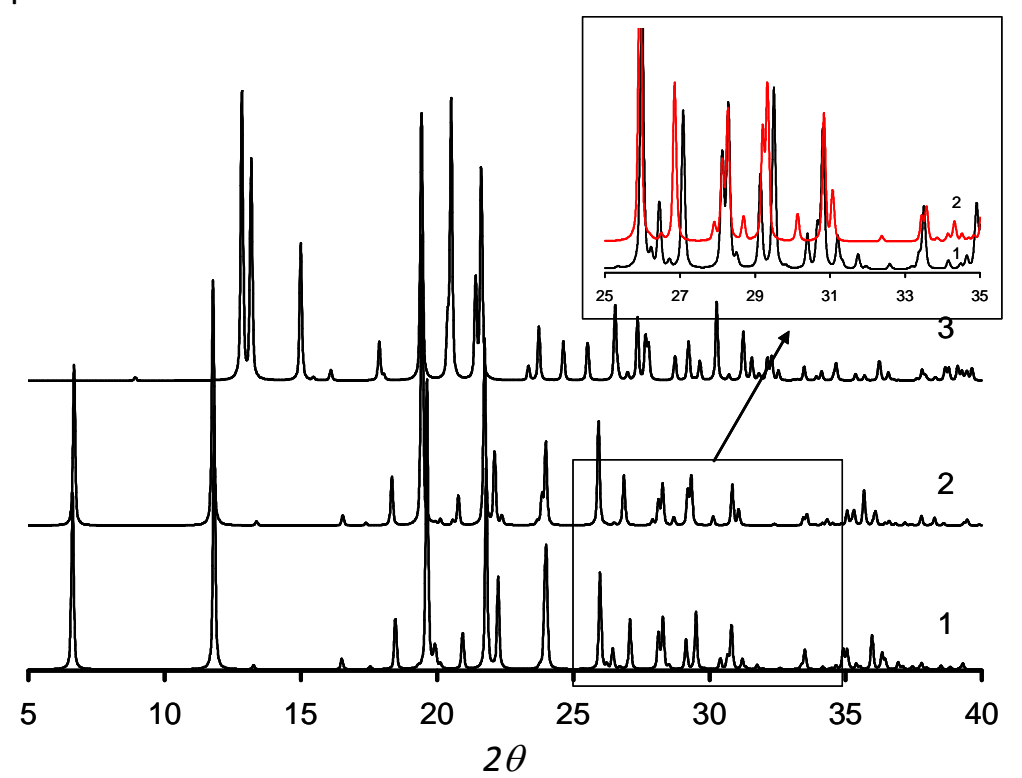

Figure 12. Powder X-ray diffraction patterns of $\alpha-, \varepsilon^{\prime}-, \varepsilon$ - polymorphs of chlorpropamide. Note the similarity of the patterns of the $\alpha$ - and $\varepsilon^{\prime}$ - polymorphs at low $2 \theta$ diffraction angles; zoomed insert enlarges the diffraction patterns of 1 and 2 at higher $2 \theta$ diffraction angles, where the differences are pronounced [80].

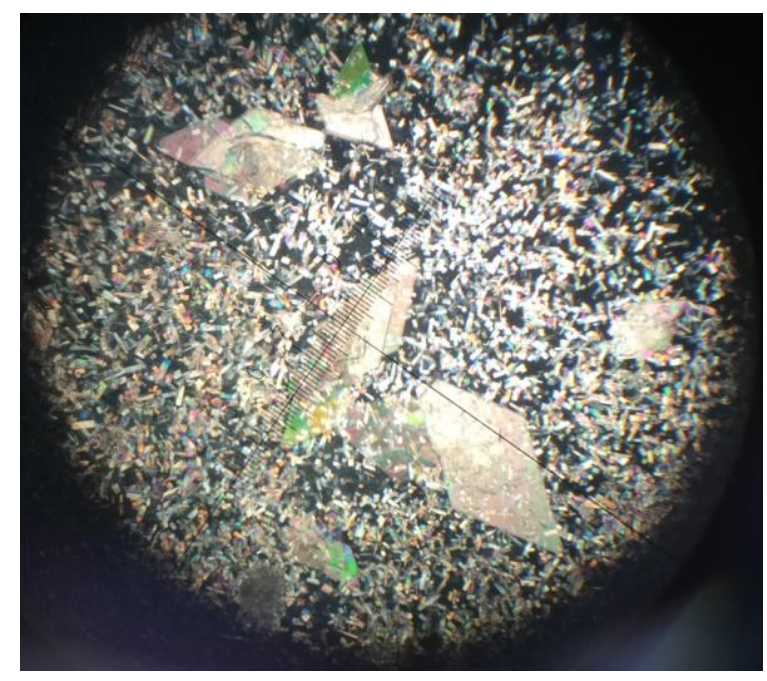

Figure 13. Recrystallization of tolazamide polymorph II (large rhombic plates) into another tolazamide polymorph I (small elongated prisms) [83].

As seen previously (cf., "Potentiometric Cycling for Polymorph Creation"), there can be features in the solubility or dissolution curves which can suggest that the composition of the solid phase does change with time, and the results at the end of the measurements refer to another phase than at the beginning. This can be further illustrated with the example of L-glutamic acid. 


\section{L-Glutamic Acid Polymorphs}

Figure 14a shows the variation of the concentration of L-glutamic acid in aqueous suspension. One can notice, that the saturated concentrations start decreasing after some time. An explanation of this phenomenon can be provided by Figure 14b, in which the relative contents of the more soluble polymorph, the $\alpha$-L-glutamic, in the solid phase in contact with solution, is plotted versus time of stirring [84].

(a) L-Glutamic Acid $\alpha$-Form Dissolution Profile

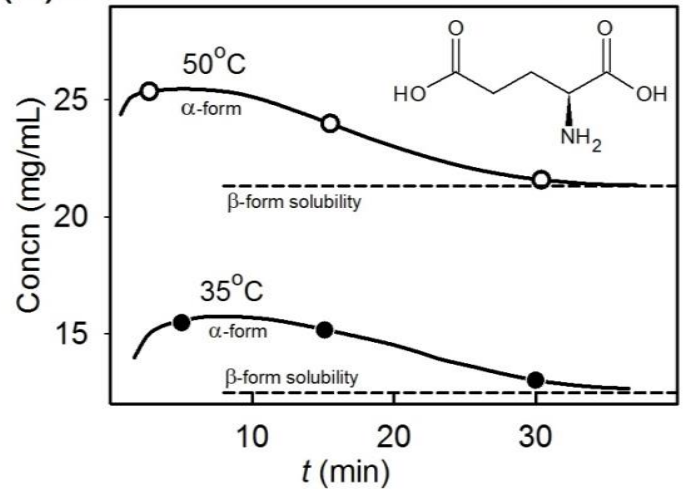

(b) L-Glutamic Acid $\alpha$-Form (\%) in Slurry vs. Time

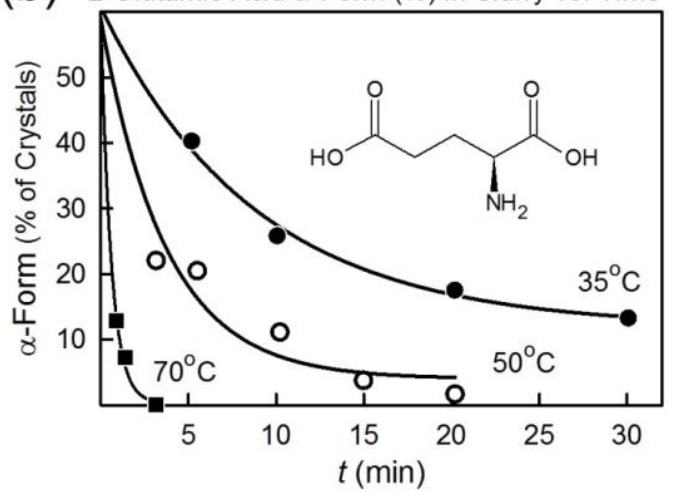

Figure 14. (a) Variation of the concentration of L-glutamic acid in aqueous solution versus time at $35^{\circ} \mathrm{C}$ and $50^{\circ} \mathrm{C}$. (b) The content of the $\alpha$-polymorph of $\mathrm{L}$-glutamic acid in the solid sample stored in aqueous solution at $35{ }^{\circ} \mathrm{C}, 50^{\circ} \mathrm{C}, 70^{\circ} \mathrm{C}$; Data from [85].

The $\beta$-L-glutamic acid is the more stable form of L-glutamic acid [84]. The $\alpha$ - to $\beta$-form transition can proceed also in the solid state under humid conditions, although very slowly (being far from completion even after several months $[84,85]$. Interestingly, no transition was observed on heating a dry crystal of the $\alpha$-form. In solution, when the crystals of the $\beta$-form precipitate, they deposit mainly on the surface of $\alpha$-crystals, rather than as single crystals suspended in solution and the two forms can fuse into each other to such an extent, that their co-existence could be revealed only by PXRD, but not by optical microscopy [84, 85].

In Figure 15 the data from Sakata [85], processed using $p D I S O L-X$, are presented in a comparison with similarly treated data from Lee et al. [86]. Figure 15a shows the expected parabolic-shaped logS-pH profile of L-glutamic acid, without indication of the polymorphic form. From the $p D I S O L-X$ analysis of the data, the intrinsic solubility, $S_{0}$, refined to $7.5 \pm 0.1 \mathrm{mg} / \mathrm{mL}$. Figure $15 \mathbf{b}$ shows the result of a single-point analysis of L-glutamic acid, known to be in the $\beta$-form [85], where the refined $S_{0}=7.7 \mathrm{mg} / \mathrm{mL}$. The comparable $S_{0}$ values suggest that Lee et al. [86] had performed measurements on the stable $\beta$-form. It is worth pointing out that on examination of the Sakata publication [85], one encounters several shortcomings in method description, which are, unfortunately, rather common when examining published results. First, solubility has been reported in units of "g solute per $100 \mathrm{~g}$ water." Given that $100 \mathrm{mg}$ of $\mathrm{NaXH} . \mathrm{H}_{2} \mathrm{O}$ plus 1 equiv. of $\mathrm{HCl}$ had been introduced to water, it is not clear, what is meant by "solute", i.e., which formula weight should be used to convert to molality. The result closest to that of Lee et al. [86] suggests that "solute" refers to the zwitterion $\mathrm{XH}_{2}$ species, not salt form, $\mathrm{NaXH} . \mathrm{H}_{2} \mathrm{O}$, that was actually added to the solution. Such ambiguities happen quite often in the nearly 850 citations in the solubility database [4]. Clarifications are often not possible, since the authors are either no longer active, or the original experimental data cannot be located, or the authors are deceased. Second, the final $\mathrm{pH}$ value was not reported [85]. For L-glutamic acid, it does not matter much, though 
knowledge of the $\mathrm{pH}_{\text {sat }}$ becomes important for low soluble molecules (e.g., mefenamic acid, diflunisal, fenbufen, indomethacin, clofazimine, terfenadine) [4]. Using $\mathrm{pDISOL-X}$, the two calculated saturation $\mathrm{pH}$ values, $\mathrm{pH}_{\text {sat }}$, are 3.38 (salt) and 3.37 (zwitterion). Third, the values of ionic strength have not been reported. Depending on which formula weight assumptions have been made, the values would be calculated as 0.54 or $0.64 \mathrm{M}$, either value well above that of normal saline.
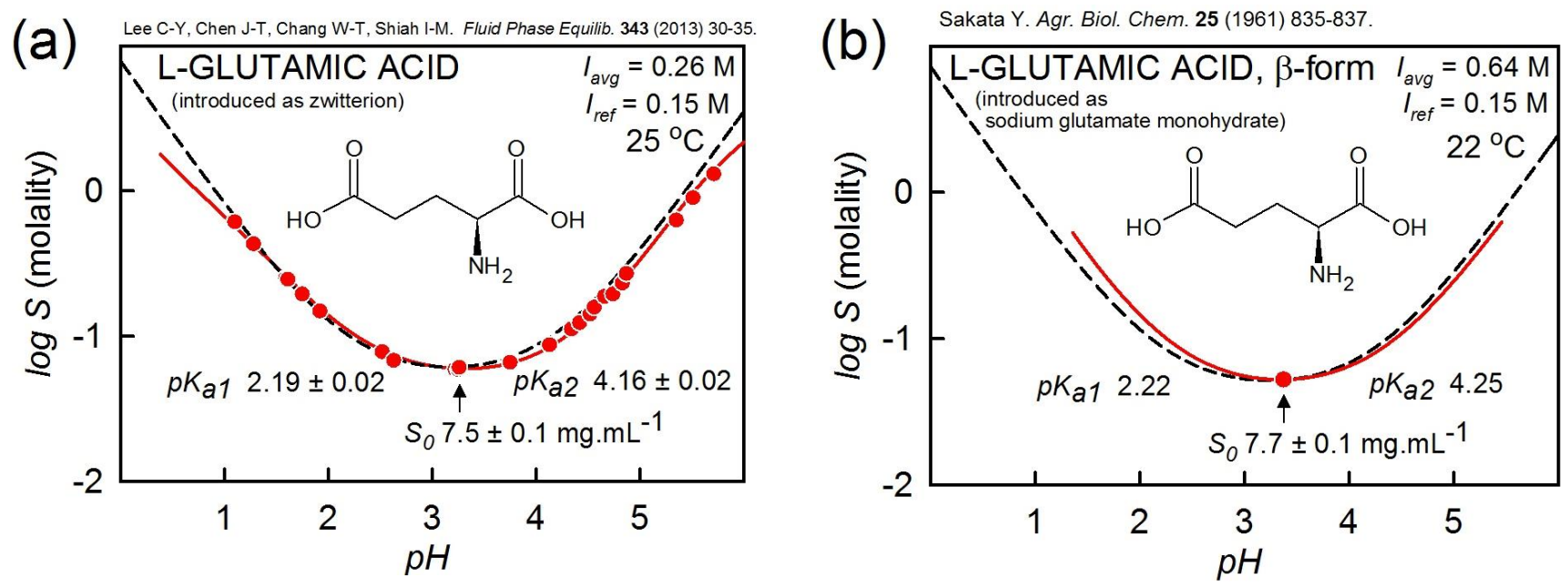

Figure 15. The data from Sakata [85] processed using pDISOL-X in a comparison with similarly treated data from [86].

\section{Cocrystals}

Interest in pharmaceutical cocrystals emerged rapidly in the last decade [30, 87-96]. Quite often cocrystals can be formed in the physical mixtures of the two components even without any action, merely due to moisture sorption by components themselves [97], or by a polymer excipient [96, 98]. Presence of cocrystals in the drug formulation is thus possible even when not anticipated. Cocrystals are often designed and produced on purpose, to increase the solubility, or the stability, or the tabletability of an active pharmaceutical ingredient (API) [30, 92, 96, 99-104]. However, when a cocrystal is dissolved, the initially high concentration of the API in solution can drop down with time, and sometimes this happens very fast. Cocrystal solubility is sensitive to coformer concentration and $\mathrm{pH}$ [31]. The presence of surfactants can have a pronounced influence on the dissolution of cocrystals. Surfactants are commonly used in pharmaceutical development, in dissolution media, as formulation aids, to enhance wetting and solubility of hydrophobic drugs [105, 106], and are, of course, encountered in vivo. Whereas the micellar solubilization of single-component crystals has been thoroughly studied, micellar solubilization of cocrystals is not well understood. A key question is: how do surfactants that solubilize the drug, influence cocrystal solubility and dissolution $[33,96]$ ? The solubility of a hydrophobic drug in aqueous solution depends on the total surfactant concentration [33]. Solubility curves of a pure drug and a cocrystal intersect at a critical stabilization concentration (CSC) point, which increases with coformer solubilization [33]. Cocrystal solubility for cocrystal R-HA in micellar solutions can be predicted from $K_{\mathrm{a}}$ and $S_{0}$ values of cocrystal components (drug, coformer) and $K_{\mathrm{sp}}$ of cocrystal in blank media [28].

Cocrystal and drug solubilities converge as they approach CSC $[83,107]$. Cocrystal CSC is highly sensitive to $\mathrm{pH}$ [108]. A key parameter, which enables the measurement of cocrystal solubility and to establish stability regions from a single experiment, is the eutectic, or transition point (Figure 16) [28]. At this point, 
$S_{\text {cocrystal }}=S_{\text {drug }}$ (cocrystal solubility in terms of drug moles); two solid phases are in equilibrium with solution; solution composition $[\mathrm{B}]_{\mathrm{tr}},[\mathrm{A}]_{\mathrm{tr}}$ is fixed at $T$ and $\mathrm{pH}$, regardless of ratio of two solid phases [28].

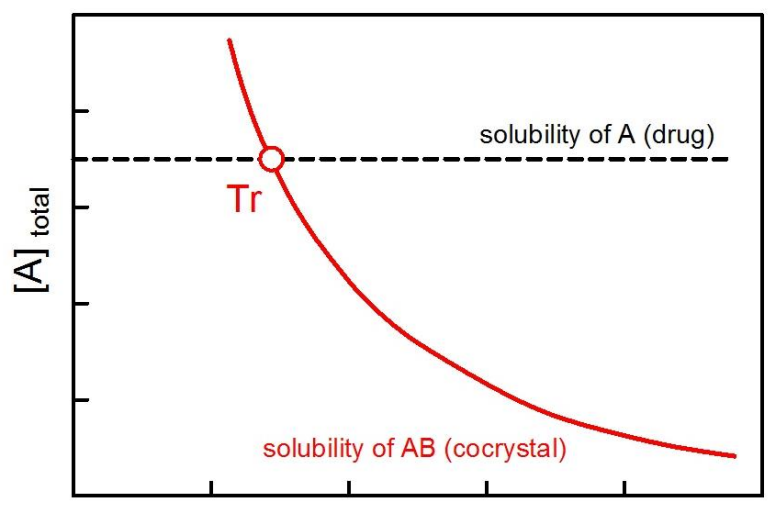

$[B]_{\text {total }}$

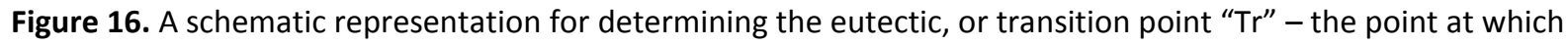
the "solubility of AB versus concentration of B" curve crosses the "solubility of A versus concentration of B" curve. At this point $S_{\text {cocrystal }}=S_{\text {drug }}$ (cocrystal solubility in terms of drug moles), and the two solid phases (AB and A) are in equilibrium with the solution. The solution composition $[B]_{t r},[A]_{t r}$ is fixed at $T$ and $\mathrm{pH}$, regardless of ratio of two solid phases. Adapted from [28].

\section{Carbamazepine-Nicotinamide Cocrystal}

Figure 17 [108] illustrates the fast drop in the carbamazepine concentration, as indicated by the shift in the UV absorption maximum, on dissolution of a carbamazepine-nicotinamide 1:1 cocrystal in water. Two minutes after the start of the dissolution of the carbamazepine-nicotinamide cocrystal, the solid sample transforms almost completely into the carbamazepine dihydrate, with a significantly lower solubility, and the concentration of carbamazepine in solution drops. Cocrystal solid-solution equilibria are dictated by solution composition. If anhydrous carbamazepine is dissolved in the solution of nicotinamide, a 1:1 cocrystal is formed [108].

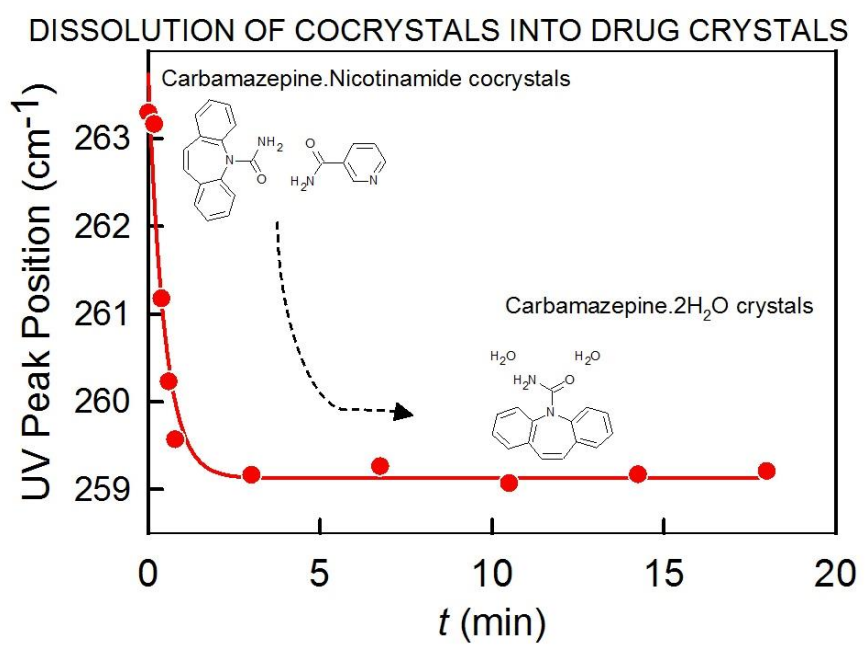

Figure 17. Transformation of a cocrystal of carbamazepine with nicotinamide into carbamazepine dihydrate on dissolution (monitored spectroscopically). Data from [108]. 


\section{Cocrystal Solubility-pH Profiles}

Cocrystals can impact $\mathrm{pH}$-dependent solubility even when the drug is nonionizable. The $\mathrm{pH}$ dependence of the solubility of a cocrystal can differ significantly from that of a pure drug compound, the curves being qualitatively different for ionizable/non-ionizable/zwitterionic API and amphoteric/basic/acidic coformer [31].

Figure 18 illustrates this using as examples the solubility of the carbamazepine - salicylic acid 1:1 cocrystal (Figure 18a) and of the carbamazepine-4 amino-benzoic acid hydrate 2:1 cocrystal (Figure 18b) as functions of $\mathrm{pH}$, and, for a comparison, the $\mathrm{pH}$ independence of the solubility of the pure drugs [31].
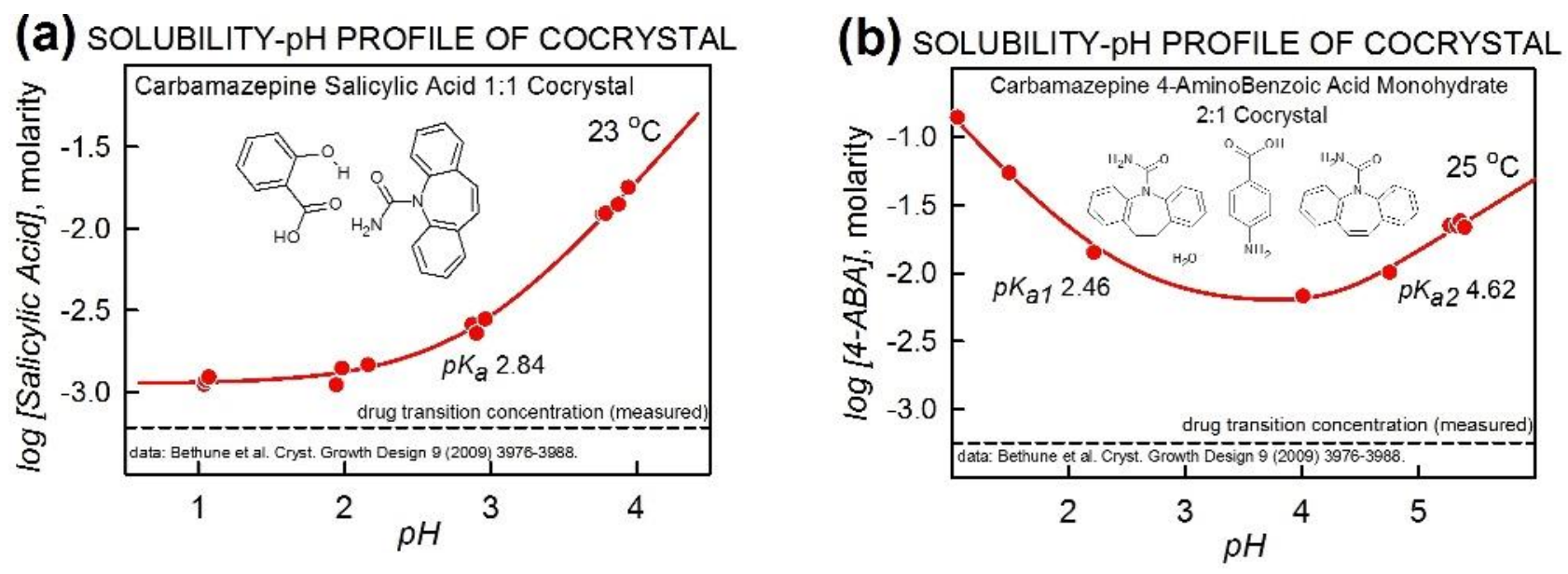

Figure 18. (a) The solubility of the carbamazepine - salicylic acid 1:1 cocrystal, and (b) the solubility of the carbamazepine - 4-aminobenzoic acid hydrate 2:1 cocrystal as functions of $\mathrm{pH}$. The dashed lines are the expected solubilities of the pure drugs [31]. The data from [31] have been processed using pDISOL-X.

The data from Bethune et al. [31] have been processed using $p D I S O L-X$. This procedure has given almost the same $K_{\text {sp }}$ value for the carbamazepine - salicylic acid 1:1 cocrystal as reported in [31], but it was necessary to introduce a complex between carbamazepine and salicylate anion. To interpret the curve with the minimum for the carbamazepine - 4-aminobenzoic acid hydrate 2:1 cocrystal, one needs to consider cocrystal dissociation and coformer ionization. 


\section{Recommendation for Solid State Characterization}

It is recommended that the solid sample for which solubility is measured be evaluated by powder X-ray diffraction (PXRD), both before and after the solubility assay. If possible, it is preferable to monitor the sample directly in solution (modern PXRD instruments specially adapted for the needs of pharmaceutical industry make this possible). If, however, such an in situ measurement is not possible, the conditions of separating the solid from solution, drying and preparing the sample for PXRD (e.g., manual grinding in a mortar) must be specified and standardized with utmost care. Quite often, phase transition, dehydration, or transformation (in a multi-component system) can occur on drying, grinding, tableting, or storage. It is recommended that the PXRD pattern be measured in a broad enough range of $2 \theta$ diffraction angles and analyzed as a whole, not just by searching selected peaks, and where possible samples should be loaded in capillaries for data collection so we minimize preferred orientation peaks.

Confocal Raman spectroscopy can be recommended as a complementary - though less unambiguous tool to control possible changes in the phase and chemical composition of the solid phase.

DSC and TGA analysis of the sample is recommended before and after the experiment. Note, that even very similar DSC curves cannot completely exclude that the samples have different phase composition.

Analysis of the final solid phase is desirable in all cases, since the form precipitating can be, and usually is, different from the form introduced at the beginning of the study, and becomes critical if some "anomalies" in the solubility-pH/time profile are observed. Careful data processing can give insights in potential changes in the phase composition during the solubility measurement, and also in the solid state. The latter can be verified by PXRD.

\section{Analysis of the Species in the Saturated Solution using Mass Spectrometry}

Mass spectrometry is a powerful technique to obtain information about the species present in any solution, including saturated solutions [53]. In solubility assays, apart from the compound itself, the presence of charged aggregates can be detected by direct infusion into the mass spectrometer of the saturated solution, as demonstrated for cefadroxyl by Shoghi et al. [53] (cf., Figure 2a). With a simple mass spectrometer (i.e., an electrospray ionization source and a single quadrupole) aggregates with a molecular mass up to 1500-2000 Da can be easily observed. In addition, the use of more sophisticated instruments, like triple quadrupoles or highresolution mass spectrometers allows the confirmation of the presence of aggregates through the product ion scan or the exact mass measurement of the aggregates.

One important requirement in the measurements when buffered solutions are used is that buffers must be MS compatible. Thus, buffers based on compounds such as formic acid, acetic acid, lactic acid, carbonic acid, ammonia, or ethylenediamine are recommended. It is helpful to report buffer concentrations in $\mathrm{mM}$ units.

Another compulsory caution is to avoid the formation of aggregates in the ionization source. This drawback can be easily overcome by raising the declustering potential of the source to high voltages (up to $\pm 200 \mathrm{~V}$, depending on the ionization mode). Under such conditions, much more fragmentation is observed, but in case aggregates are detected it is ensured that they come uniquely from the sample itself. 
Although MS provides a clear view of the solutes in solution, it is important to notice that not all type of compounds are susceptible to be ionized in certain conditions (especially in absence of organic solvents). Other complementary techniques should be considered for solution characterization of those compounds that do not ionize easily, form neutral aggregates, or have a high aggregation number such as micelles, among others.

\section{Types of Solubility}

Water Solubility, $S_{w}$, and its Relationship to Intrinsic Solubility, $S_{0}$

The solubility in water, $S_{w}$, is frequently reported for ionizable druglike substances. The procedure calls for adding an unspecified "excess" solid (ideally, the free-acid or free-base, not the salt) to distilled water, followed by the measurement of the substance concentration after equilibration. Frequently, $\mathrm{pH}$ of the saturated solution, $\mathrm{pH}_{\text {sat, }}$ is not reported (and probably not measured). Usually, the reported experimental details are incomplete, and so such measurements can have substantial uncertainty. It is useful to consider the relationship between $S_{w}$ and the intrinsic solubility, $S_{0}$, the value an ionizable molecule indicates at the $\mathrm{pH}$ where the molecule is completely uncharged $\left(\mathrm{pH}<<\mathrm{p} K_{\mathrm{a}}\right.$ for acids or $\mathrm{pH}>>\mathrm{p} K_{\mathrm{a}}$ for bases; cf., Appendix $\left.\mathrm{B}\right)$.

When a relatively soluble weak acid/base is added to water, the $\mathrm{pH}$ of the suspension is altered by the ionizing molecule, in the direction where the molecule remains largely in the uncharged form: $S_{\mathrm{w}} \approx S_{0}$, provided the compound is added as a pure free acid/base. For compounds added as salts, it is frequently not possible to deduce $S_{0}$ and $\mathrm{pH}_{\text {sat }}$ from just $S_{\mathrm{w}}$ and $\mathrm{p} K_{\mathrm{a}}$, since the total amount of added compound can affect the disposition of the saturated solution: if not enough of the drug salt is added (i.e., salt solubility product not exceeded), the solid disproportionates to the free acid/base in the saturated solution, with $\mathrm{pH}_{\text {sat }}$ depending on the weight of drug salt added [11].

If the ionizable compound is practically insoluble, then the measured value of $S_{w}$ can be quite different from $S_{0}$, since not enough of the compound dissolves to alter the $\mathrm{pH}$ in the direction of maintaining a nearly uncharged molecule in the poorly-buffered solution. If the $\mathrm{p} K_{\mathrm{a}}$ is known and the Henderson-Hasselbalch $(\mathrm{HH})$ equation is valid in the particular case, it is possible to calculate the $\mathrm{pH}_{\text {sat }}$, as well as $S_{0}$. For a one- $K_{\mathrm{a}}$ molecule, $\log S=\log S_{0}+\log \left[10^{ \pm(\text {pHsat-pKa })}+1\right]$ (' \pm ' is ' + ' for an acid and '-' for a base). Additional Henderson-Hasselbalch equations for ampholytes and multiprotic acids and bases are tabulated in Table B.1 in Appendix B [21, 27].

When measured compounds contain protogenic impurities, the $\mathrm{pH}_{\text {sat }}$ may be affected substantially under poorly-buffered conditions, which could lead to a change in the measured $S_{w}$. Under such circumstances, the calculation of $S_{0}$ from $S_{w}$ may be quite inaccurate.

Abraham and Le [109] discussed the relationship between the measured $S_{w}$ and $S_{0}$, and identified under which circumstances large differences are expected between the two values. The authors derived useful plots of $S_{0} / S_{\mathrm{w}} v \mathrm{~s} . \log S_{\mathrm{w}}$ for acids and bases over a range of $\mathrm{p} K_{\mathrm{a}}$ values. For example, for ionizable acids with $\mathrm{p} K_{\mathrm{a}} 5$ and $S_{\mathrm{w}}>0.001 \mathrm{M}$, or with $\mathrm{p} K_{\mathrm{a}} 3$ and $S_{\mathrm{w}}>0.1 \mathrm{M}$, the water solubility is practically equal to the intrinsic solubility. The same is true for ionizable bases with $\mathrm{p} K_{\mathrm{a}} 10$ and $S_{\mathrm{w}}>0.01 \mathrm{M}$, or $\mathrm{p} K_{\mathrm{a}} 8$ and $S_{\mathrm{w}}>0.0001 \mathrm{M}$.

When the aqueous solubility of practically insoluble free bases $\left(\mathrm{p} K_{\mathrm{a}}>9\right)$ is measured in water, the $\mathrm{pH}$ is only slightly affected by the minute extent to which the base dissolves. To a much greater degree, the measured $\mathrm{pH}$ is regulated by a much stronger buffer present in water, namely, dissolved carbon dioxide. This is often not 
factored in. In the (unlikely) absence of $\mathrm{CO}_{2}$, the drug-saturated solution may be at $\mathrm{pH}>9$. But the presence of dissolved $\mathrm{CO}_{2}$ can lower the $\mathrm{pH}<6$, depending on the level of $\mathrm{CO}_{2}$ and how insoluble the basic compound is. Rytting et al. [110] reported $\log S_{\mathrm{w}}=-4.87$ for terfenadine dissolved in water, with no further information. If ambient $\left[\mathrm{CO}_{2}\right]=\{0,10,20\} \mu \mathrm{M}$, the calculated $\log S_{0}=\{-5.7,-6.3,-8.3\}$ and $\mathrm{pH}_{\text {sat }}=\{9.2,8.5,6.6\}$, respectively. Consequently, the error in the calculated $S_{0}$ of practically insoluble basic drugs in water is expected to be enormous (up to 3 log units), since it is very difficult to eliminate $\mathrm{CO}_{2}$ entirely simply by bubbling an inert gas $\left(\mathrm{Ar}, \mathrm{N}_{2}, \mathrm{He}\right.$ ) through the solution. Furthermore, any protogenic drug impurity in water under the circumstances would also lead to large uncertainties in the $\mathrm{HH}$-calculated $S_{0}$. That is why one ought to actually measure the $\mathrm{pH}$ of the saturated solution. But, measuring the $\mathrm{pH}$ accurately of an unbuffered solution is another problem, due to the effects of uncontrolled electrode junction potentials and possibly inappropriately calibrated $\mathrm{pH}$ electrodes [27]. The addition of a background electrolyte (e.g., $0.15 \mathrm{M} \mathrm{NaCl}$ ) would lessen the junction potential error.

\section{Solubility in a Single Buffered Solution, $S_{p H}$}

The use of a buffered solution lessens some of the uncertainties associated with $\mathrm{pH}$ measurement and the impact of trace impurities. The $\mathrm{pH}$ of the saturated drug solution still needs to be carefully measured after equilibration. Still, to convert the $S_{\mathrm{pH}}$ to the intrinsic value, $S_{0}$, it is necessary to know the independentlymeasured $\mathrm{p} K_{\mathrm{a}}$ and the application of the $\mathrm{HH}$ equation (provided it is valid for the studied molecule).

\section{Intrinsic Solubility, $\mathrm{S}_{0}$, from Multiple logS-pH Measurements}

When critically comparing the solubility of different molecules, the intrinsic value is the appropriate one to consider. The most general way to determine the intrinsic solubility, $S_{0}$, requires multiple $\mathrm{pH}$ measurements using the SF method. Ionizable/nonionizable drugs may be considered, as well as reactions that form drug salts, complexes, aggregates/micelles, or cocrystals. CheqSol is also a good way to determine intrinsic solubility of ionizable drugs. Interferences due to buffers, salts, or complexing agents are generally avoided. Several of the preceding case studies illustrate how complicated the analysis of logS-pH can be. When measuring several different $\log S-\mathrm{pH}$ points, both below and above the $\mathrm{p} K_{\mathrm{a}}$ (and $\mathrm{p} K_{\mathrm{a}}^{\text {Gibbs }}$ with drug salt precipitates), it is not necessary to rely on simple $\mathrm{HH}$ equations (which might not accurately predict the logS dependence on $\mathrm{pH}$ ). Buffers may be required to cover the regions of $\mathrm{pH}$ poorly buffered by the drug molecule. Also, buffers may facilitate the uniform $\mathrm{pH}$ adjustments of the solutions using standardized titrants.

\section{Other Solubility Terms Used in the Literature}

Other solubility terms (e.g., biological, native, natural, unbuffered, turbidimetric, kinetic) are used in the literature. We encourage practitioners to give careful consideration to the meaning of the terms used to associate with solubility. At times these lesser-common terms have been used without critical definition or sufficiently rigorous measurement practices. 


\section{Recommendations for Type of Solubility to Measure}

Generally, the measurement of the water solubility, $S_{w}$, cannot be recommended. If there is good reason to do so, then (a) use freshly distilled/purified water that had been allowed to equilibrate at the measurement temperature, (b) measure the $\mathrm{pH}$ of the water exposed to ambient air, $\mathrm{pH}_{\mathrm{w}}$, before adding the sample (to estimate $\mathrm{CO}_{2}$ concentration), and (c) measure the final $\mathrm{pH}_{\text {sat }}$. Sample purity needs to be verified.

Potentiometric methods such as CheqSol are well suited to determine intrinsic solubility (avoiding the possible interferences due to buffers), and can thus be well recommended.

Multiple-pH shake-flask assays are recommended for general applications, since many different solution properties can be revealed by the logS-pH profiles. A single solubility determination at a measured $\mathrm{pH}$ can establish the intrinsic solubility. But it is recommended that at least three $\mathrm{pH}$ points be measured if the intrinsic solubility is to be determined reliably: $\mathrm{pH}=\left\{\mathrm{p} K_{\mathrm{a}}-2, \mathrm{p} K_{\mathrm{a}}, \mathrm{p} K_{\mathrm{a}}+2\right\}$, where $\mathrm{p} K_{\mathrm{a}}$ is independently determined. If also the salt solubility is being determined, then more points would be required: e.g., $\mathrm{pH}=\left\{\mathrm{p} K_{\mathrm{a}}{ }^{\text {Gibbs }}-2, \mathrm{p} K_{\mathrm{a}}{ }^{\text {Gibbs }}, \mathrm{p} K_{\mathrm{a}}^{\text {Gibbs }}+2\right\}$, in addition to those points around the $\mathrm{p} K_{\mathrm{a}}$. The characterization of drug salts, aggregates/micelles, complexes, and cocrystals generally requires multiple-pH solubility data, in thoughtfully-designed assays.

\section{Temperature}

Solubility is a function of temperature, so the assay temperature always needs to be reported. It is advisable to measure and record the actual temperature in the sample vessel during the equilibration period. Or better yet, the measurement is performed in a thermostated vessel kept at $25 / 37^{\circ} \mathrm{C}$. It is clear that unspecified "room temperature" can be different from laboratory to laboratory, and in some cases, seasonally variable.

When solubility measurements are conducted at different temperatures, it is common to report the solubility values in mole fraction, mass fraction, and molality units, since these do not depend on the density of the solutions, which can change with temperature. Since advanced computational approaches to evaluate the logS-pH data are anchored in molarity units, it would be useful to report the weight of a certain volume of the saturated solution at the assay temperature, after the solid had been separated from it, so that molality units can be converted to those based on molarity.

\section{Recommendation for Reporting Temperature}

The temperature must always be measured at the time equilibration is established, and its value reported in the publication. The method of temperature control should be reported. It is not helpful to state the value as "room temperature." 


\section{lonic Strength}

Measured solubility can be affected by ionic strength (especially when salt solubility is measured), so the ionic strength usually needs to be reported. The average total ionic strength needs to be calculated from the contributions at each $\mathrm{pH}$ point. It is the $\mathrm{pH}$-dependent sum of the contributions of inert background electrolytes ( $\mathrm{NaCl}, \mathrm{KCl}$, etc.), buffer constituents, as well as the sample (especially when introduced in salt form). It is not possible to back-calculate the ionic strength at each pH point if the report simply states that "buffers were used" or "measurement was done in water."

\section{Recommendation for Reporting lonic Strength}

The total ionic strength, averaged over the $\mathrm{pH}$ range of the assay, needs to be reported.

If the computer program used to evaluate the $\log S-\mathrm{pH}$ data does not calculate the ionic strength, then the report must give enough information about the solution composition so that the average total ionic strength can be independently calculated - i.e., precisely state: (a) concentration and type of ionic strength adjustor used (e.g., $0.15 \mathrm{M} \mathrm{NaCl}$ ), (b) precise stoichiometry and weights of buffer used (e.g., $\mathrm{Na}_{2} \mathrm{HPO}_{4} \cdot 12 \mathrm{H}_{2} \mathrm{O}$, not just "sodium phosphate"), (c) weight and form of sample used (e.g., amiodarone hydrochloride), (d) any other relevant electrolytes that would contribute to the ionic strength calculation.

\section{Measurement of pH using Glass Electrodes}

A pH electrode is a sensitive electrochemical probe that can indicate the degree of acidity of a solution. The device is a combination of a reference half-cell (in a compartment separated from the test solution by a porous-barrier junction that permits the flow of an ionic current but prevents convective mixing of the reference and the test solutions) and a pH-sensing glass electrode half-cell. The two half cells immersed in the test solution complete the electrical circuit, as a high impedance (i.e., extremely low electrical current) voltage is measured, which is proportional to $\mathrm{pH}$ of the solution. Such a system needs to be calibrated against solutions of defined $\mathrm{pH}$, so that the measured voltage can be converted to the "operational" $\mathrm{pH}$ scale. The latter is nearly, but not precisely, the activity scale, $\mathrm{p}_{\mathrm{a}} \mathrm{H}$ [27]. The relationship between voltage and the operational $\mathrm{pH}$ depends on temperature and ionic strength.

For common laboratory use, the pH electrodes are typically calibrated against standard ("NIST-traceable") pH 4.00 (phthalate) and 7.00 (phosphate) buffers, with ionic strength of $0.10 \mathrm{M}$. However this procedure may be inadequate for specialized applications, such as $\mathrm{p} K_{\mathrm{a}}$ determination and solubility-pH measurement. Solubility determination may involve measuring $\mathrm{pH}$ in solutions with near zero ionic strength or ionic strength as high as $1 \mathrm{M}$, far from the $0.1 \mathrm{M}$ value of the calibrating buffers. The reference electrode part of the circuit can develop significant porous-barrier junction voltages ("liquid junction potentials," LJP) when immersed in solutions with near zero ionic strength or with $\mathrm{pH}<2$. Also, the glass bulb of the sensor can develop mechanical stress when dipped in strongly acid solutions $(\mathrm{pH}<1)$. Both effects contribute to an error in $\mathrm{pH}$ reading, which can be as high as a $\mathrm{pH}$ unit [4].

In published solubility studies, the electrode calibration is virtually never described and the brand of 
electrode used is largely unidentified. In contrast, attention to such detail is the norm in $\mathrm{p} K_{\mathrm{a}}$ determination practice, where specialized calibration methods are well developed [22, 27, 44, 45, 111-113]. Researchers who determine $\mathrm{p} K_{\mathrm{a}}$ values using commercial analyzers are well acquainted with the four-parameter electrode standardization procedure, described in Appendix C. Those using the Sirius Analytical titrators will recognize the procedure as the "Four-Plus" method, and those using the Pion titrators will recognize it as the "ABC" method.

\section{Recommendations for $\mathrm{pH}$ Electrode Calibration}

It is recommended that research-grade combination $\mathrm{pH}$ glass electrodes be used. It is a good idea to identify the brand and make of the electrode. The calibration procedure used needs to be concisely described. Ideally, the four-parameter electrode standardization procedure is recommended [45]. Simpler procedures may be satisfactory, as long as they are described precisely.

The reported $\mathrm{pH}$ should be on the "operational" pH scale. The standardization procedure can be used to convert $\mathrm{pH}$ to the "concentration" scale, $\mathrm{p}_{\mathrm{c}} \mathrm{H}$, which is used in equilibrium quotients, as described in Appendix C.

Particular attention should be given to the $\mathrm{pH}$ electrode calibration when extreme $\mathrm{pH}$ is measured $(\mathrm{pH}<1$ or $\mathrm{pH}>12)$ or when the ionic strength reaches high values, as in the case of salt solubility measurement, or near zero values (in unbuffered solutions).

\section{Buffers}

A pH buffer is a solution which resists $\mathrm{pH}$ change when a small quantity of an acid or a base is added to it. For example, a $50 \mathrm{mM}$ phosphate buffer solution at $\mathrm{pH} 6.8$ has a "buffer capacity" of $27 \mathrm{mM} / \mathrm{pH}$. That is, to change $\mathrm{pH}$ of a $1 \mathrm{~mL}$ buffer solution from 6.8 to 6.7 , it would take $5.4 \mu \mathrm{L}$ of $0.5 \mathrm{M} \mathrm{HCl}$. Distilled water has the buffer capacity $<0.005 \mathrm{mM} / \mathrm{pH}$. So, to change the $\mathrm{pH}$ of distilled water from 6.8 to 6.7 would require $<0.001 \mu \mathrm{L}$ of $0.5 \mathrm{M} \mathrm{HCl}$. The minimum volume addition from dispensers in commercial $\mathrm{p} K_{\mathrm{a}}$ analyzers would produce at least a $\mathrm{pH} 2.1$ jump in the $\mathrm{pH}$ 5.8-8.4 interval in distilled water.

A surprising number of investigators prepare a standard buffer solution of a known $\mathrm{pH}$, but then neglect to measure the actual $\mathrm{pH}$ of the drug-buffer slurry when equilibrium is reached. In common practice, buffers can be used to set the $\mathrm{pH}$ of an assay solution to a desired value. The $\mathrm{pH}$ change due to the introduction of drug is not zero, however. It depends on the capacity of the buffer solution and the ionization characteristics and amount and solubility of the added drug. Even with a buffer solution, the $\mathrm{pH}$ can change by several units upon the addition of an ionizable substance. See Table 1 for examples.

\section{Adjusting $\mathrm{pH}$ in Multi-pH Solubility Measurement using a Titrant}

There are many different ways to prepare multiple- $\mathrm{pH}$ solutions in solubility applications. The $\mathrm{pH}$ of the solubility medium can be modified using a standardized titrant, which can be a strong electrolyte, $\mathrm{HCl} / \mathrm{NaOH}$, $\mathrm{CH}_{3} \mathrm{SO}_{3} \mathrm{H}, \mathrm{CF}_{3} \mathrm{COOH}$, or a moderate electrolyte, like $\mathrm{CH}_{3} \mathrm{COOH}, \mathrm{H}_{3} \mathrm{PO}_{4}$, tartaric acid, L-lysine, etc. Even though a buffer of a known $\mathrm{pH}$ is used, let's accept that the final $\mathrm{pH}$ of a fully-equilibrated drug-saturated solution has to 
be actually measured.

One way (the most popular) is to look up standard buffer recipes and make the buffers to the desired $\mathrm{pH}$. (Many of these prepared buffer solutions are commercially available.) This may seem like a time-saving approach, but the choices of buffers may be inappropriate and may make the analysis of the logS-pH profile an unnecessary challenge. If many different ingredients are selected to cover the $\mathrm{pH}$ range, instances of drugbuffer interactions may be problematic (Figure 2a, [53]). Choosing concentrated buffer solutions can lead to greater drug-buffer interactions and excessively elevated ionic strengths in the final solutions. Drug-buffer complexes raise solubility, while drug-buffer precipitates decrease solubility. Some buffers, such as phosphate are not compatible with mass spectrometry [53]. Some buffers have too high a UV absorbance. Boric acid buffers can covalently interact with 1,2-diol portions in drug molecules. Some combinations of buffers have cross interactions: e.g., phosphate and ethylenediamine buffers can form ion-pairs [45]. The optimal choice of buffers requires some experience.

Another way (less popular) is to avoid buffers altogether. After all, the ionizable drug being studied can act as its own buffer, particularly if the drug molecule has multiple $\mathrm{p} K_{\mathrm{a}}$ values. So, self-buffered solutions with excess solid can be adjusted to different values of $\mathrm{pH}$ by additions of $\mathrm{HCl}$ or $\mathrm{NaOH}$. This is actually not a bad approach, since interferences due to drug-buffer interactions are eliminated. The downside of this approach is that some sparingly soluble drugs may have virtually no buffer capacity over a wide $\mathrm{pH}$ range, so that impurities may have an inordinate sway over the equilibrated $\mathrm{pH}$. Not using auxiliary buffers would not be recommended when studying practically insoluble bases with $\mathrm{p} K_{\mathrm{a}}>8$ (e.g., terfenadine, chlorpromazine, etc.), since reading and adjusting $\mathrm{pH}$ may be problematic experimentally.

The third way (occasionally used) is to start with a "universal" buffer mixture of several individual buffer components. This mixture can be very beneficial, since the buffer capacity remains above a certain minimum level across the whole $\mathrm{pH}$ range. For example, the Britton-Robinson buffer (acetic acid, phosphoric acid, boric acid, $40 \mathrm{mM}$ each) has an initial $\mathrm{pH} 1.9$ [22]. It is quite simple to adjust the $\mathrm{pH}$ to any higher value, simply by adding a known amount of $0.5 \mathrm{M} \mathrm{NaOH}$ (titrant).

Constancy in ionic strength across the $\mathrm{pH}$ range of interest is also desirable, but most universal buffer formulations fall short of that goal (cf., Appendix A). Most investigators using universal buffers pick them from well-tested published recipes. (Commercial sources of such solutions are not common.) Since the universal buffers were not specifically designed for solubility measurements, their characteristics may not be optimal, for the same reasons as mentioned above (drug-buffer interactions, excessive ionic strength, excessive computational complexity in data analysis, etc.).

The fourth way (newly explored and quite promising) is to take the best of the idea of universal buffers, but reduce the factors leading to drug-buffer complications noted above. Such new buffers may be called "Minimalist Universal Buffers" (MUB).

\section{Minimalist Universal Buffer (MUB) Formulation}

Traditionally, universal buffer design required a lot of experiments to be performed to come up with the desired formulation. Predicting the characteristics of universal buffers computationally would have been quite a challenge then. Now that such computational approaches are possible, universal buffer designs specific to 
solubility-pH applications can be explored easily. In the recent study of the solubility-pH of terfenadine, a MUB made of lactic acid and ethylenediamine was tested successfully [114].

In a solubility-pH assay, only the regions low in buffer capacity need a boost from an auxiliary buffer. It can be computationally predicted (e.g., $p$ DISOL-X) what buffer capacity the drug itself contributes to the solution in the $\mathrm{pH}$ range of interest [22-25]. The $\mathrm{pH}$ regions of unacceptably low buffer capacity can suggest which auxiliary buffer to use. Without the capacity boost, it would be impractical to add the extremely small volumes of the $\mathrm{NaOH} / \mathrm{HCl}$ titrant to adjust the $\mathrm{pH}$ in 0.2 increments across the low-capacity region.

In the terfenadine example [114], two such regions were identified. A MUB consisting of just two components, $10 \mathrm{mM}$ lactic acid and $10 \mathrm{mM}$ ethylenediamine, filled in the buffer capacity holes. Terfenadine lactate was not expected to precipitate, since the lactic acid concentration was low enough. $\mathrm{HCl}$ was used to lower the $\mathrm{pH}$ to 2 , and the chloride salt of terfenadine was characterized in the $\mathrm{pH} 2-5$ region. The ionic strength was kept well under control.

Figure $\mathbf{A} \mathbf{3}$ in Appendix A shows the region above $\mathrm{pH} 5$ for haloperidol where ethylenediamine was a useful added buffer. There, the steepness in the titration curve was lessened by the added buffer. For haloperidol without such a capacity boost, the reading of $\mathrm{pH}>5$ would have been a challenge due to low ionic strength and the concomitant junction potential effects. Also, adjusting $\mathrm{pH}$ in increments of 0.2 in that region would not have been feasible with typical dispensers and titrant concentrations.

\section{Recommendations for Buffer Use}

Multiple-pH assays using buffers are recommended for general applications. In reporting solubility-pH results, state precisely which buffers were used: in which salt forms and at what concentrations. This is needed information for calculating the total ionic strength at each point. Note that the use of a high capacity buffer does not eliminate the need to measure the equilibrium $\mathrm{pH}$ of a saturated solution.

Sufficient buffering is needed so that $\mathrm{pH}$ can be measured reliably and that the titrant can be dispensed precisely to set the desired $\mathrm{pH}$. Ordinarily, relatively low buffer concentrations (e.g., 5-25 mM) can be recommended. Use of higher concentrations need to be critically justified. The sample itself may be an adequate buffer. It is not beneficial to overload the assay protocol with added complications arising from unintended buffer-drug interactions. The use of universal buffers (e.g., Britton-Robinson, Sorensen, Mcllvaine - cf., Appendix A) can be very convenient, but one must guard against unintended/unnecessary complications. Some buffer components may cause difficulties in UV or mass spectrometry measurement. The MUB approach merits further considerations, since many of the down-sides of buffer use can be designed out.

\section{Ionization Constant $\left(p K_{\mathrm{a}}\right)$}

High-quality $\log S-\mathrm{pH}$ data are measured so that a reliable value of the intrinsic solubility, $S_{0}$, as well as of equilibrium constants of any self-aggregates, micelles, drug-buffer complexes, and of the salt or cocrystal solubility products, $K_{\mathrm{sp}}$, can be determined. The $\mathrm{p} K_{\mathrm{a}}$ is required in the calculations. There were several 
examples in the case studies of how critical it can be to know the accurate $p K_{\mathrm{a}}$. The temperature associated with the $\mathrm{p} K_{\mathrm{a}}$ needs to match that of the solubility assay. Also, the "reference" ionic strength, $I_{\text {ref, }}$ in data analysis needs to be set equal to the ionic strength at which the $\mathrm{p} K_{\mathrm{a}}$ was determined.

In the older literature, sometimes the $\mathrm{p} K_{\mathrm{a}}$ had been determined from logS-pH data $[49,50]$. The required assumption is that the Henderson-Hasselbalch accurately describes the log $S$-pH curve. The assumption is not valid in many cases, and thus the method can result in large systematic errors in the $\mathrm{p} K_{\mathrm{a}}$ value (cf., Figure $\mathbf{2 c}$ ).

It is far more reliable to use purpose-designed $\mathrm{p} K_{\mathrm{a}}$ measurement techniques (e.g., potentiometric, spectrophotometric, or capillary electrophoretic), under conditions where ionic strength is well controlled and complications due to sample complexation, self-aggregation, or precipitation are demonstrably avoided. It is felt that commercially-available $\mathrm{p} K_{\mathrm{a}}$ prediction programs are not accurate enough for use in the interpretation of experimental logS-pH data.

It is quite important that when the $\mathrm{p} K_{\mathrm{a}}$ of a sparingly-soluble drug is determined at $37{ }^{\circ} \mathrm{C}$ using the cosolvent extrapolation method, that methanol not be used as the organic solvent. It is highly volatile, and systematic errors can confound the determination, which sometimes can be difficult to recognize. A suitable solvent alternative is n-propanol. However, if the $\mathrm{p} K_{\mathrm{a}}$ assay can be performed rapidly $(<3-5 \mathrm{~min})$, the amount of methanol lost may be small at $37{ }^{\circ} \mathrm{C}$. (When using methanol-water solutions, it could be prudent to measure the final solution volume, e.g., by weighting the vial before and after the assay.)

\section{Recommendations for $p K_{a}$ Determination}

The determination of the $\mathrm{p} K_{\mathrm{a}}$ solely from solubility-pH data cannot be recommended.

It is recommended that methods specifically designed to determine $\mathrm{p} K_{\mathrm{a}}$ of very poorly soluble molecules be used (e.g., state-of-the-art UV spectrophotometry, capillary electrophoresis, potentiometry). These methods are widely available and have been fine-tuned for the challenge. Commercial $\mathrm{p} K_{\mathrm{a}}$ instruments based on the above three technologies are generally well-supported by their manufacturers.

\section{Equilibration Time and Stirring Protocol}

One of the most important experimental conditions of determination of equilibrium solubility is that in the solubility suspension, the system has to achieve the equilibrium state. That is, when the dissolution rate of the solid is equal to its precipitation rate from the solution. This dynamic equilibrium can be maintained only by the presence of solid in the system. Perturbation, like filtration can invalidate this condition.

The optimal time of equilibration (also called incubation time) in the SF method is difficult to specify and is often a debated topic. The equilibration time is mainly determined by the dissolution rate of the compound, which depends on many factors like morphology, crystallinity, and particle size. The fine micro-crystals dissolve more quickly than large ones; amorphous solids dissolve faster than crystalline substances. The optimal time valid for every compound cannot be specified. So the best practice in the most rigorous application of the SF method is to check the required incubation time from compound to compound. However, in a standard protocol suggested for routine measurement, a generally accepted equilibration time would be useful. In a 
comprehensive study - among other factors - the effect of incubation time was tested on the equilibrium solubility of model compound, hydrochlorothiazide [3]. The achievement of equilibrium was divided into two different steps: vigorous agitation of solubility suspension with stirring and sedimentation. First, the time of stirring was varied between 0.5 and $48 \mathrm{~h}$, followed by constant time sedimentation. Second, after $48 \mathrm{~h}$ of constant stirring time, the sedimentation was varied between 1 and 24h (Figures 2 and 3 in [3]). The study revealed that sedimentation is an indispensable part of incubation, because during the intensive stirring, a supersaturated solution can form, which needs adequate time in a resting state to form the true equilibrium. Based on these results $24 \mathrm{~h}$ incubation time ( $6 \mathrm{~h}$ stirring followed by $18 \mathrm{~h}$ sedimentation) was suggested as a general protocol.

It may be noted that practically insoluble compounds which consequently have very slow dissolution rates may require much longer dissolution time. The unusually high standard deviation of solubility obtained by the above standard protocol may be an indicator of the problem. In such case, a series of repeated measurements with increasing agitation time would be necessary until the solubility is no longer changing. Some reported extremely long equilibration time (e.g., 10-30 d) set up several problems: decomposition of the sample, molding of the buffer, changing the $\mathrm{pH}$, evaporation, difficulty in temperature control, etc. Methods shortening the incubation time are known but not widely used in the practice [115].

\section{Recommendations for equilibration time}

The use of intensive stirring for agitation followed by sedimentation to reach the solubility equilibrium is recommended. The optimal speed of the stirrer depends on the volume of solubility suspension and the characteristics of the solid. It should provide high surface contacts of phases and avoid the formation of stable colloid system.

As a good average time of incubation, $24 \mathrm{~h}$ ( $6 \mathrm{~h}$ stirring and $18 \mathrm{~h}$ sedimentation) can be recommended for overwhelming majority of compounds. In case of very sparingly soluble compounds, it is recommended that the suitability of the selected equilibration time be verified. It is reasonable to start with a $6 \mathrm{~h}$ stirring time, and check whether the sample concentration is continuing to decrease at longer times.

\section{Separating Solid from Saturated Solution}

Three alternative techniques (sedimentation, centrifugation, and filtration) can be used for the separation of the solid from the saturated solution before aliquots can be taken out for concentration measurement. A recent survey [4] showed that in majority of papers the means of separation used has not been indicated. Where even declared, the filtration was the most frequent method (44\%), while centrifugation (14\%) and sedimentation (9\%) were less used.

In the shake-flask method, sedimentation is the primary method of choice, because it assures the precondition of dynamic solubility equilibrium, without disrupting the heterogeneous system (see above). At the same time, direct sampling from the clear supernatant is simple and fast. The aliquots taken out must be completely transparent, and free of any solid particles. If compound properties hinder the formation of clear 
solution upon sedimentation resulted in opalescent solution, centrifugation can be the alternative separation method. For such samples centrifugation at $1000 \mathrm{~g}(\sim 2000 \mathrm{rpm})$ for $10 \mathrm{~min}$ can be suggested. Procedure should be repeated if necessary.

\section{Surface Adsorption by "Just-Saturated" Sample Solution}

For some compounds the adsorption to vials (glass or plastic) is a problem, especially with lipophilic bases and neutral molecules in weakly buffered solutions, as described by Palmgrén et al. [116]. It can be viewed as a partitioning phenomenon. Pretreatment of the vial surfaces with subsequently-discarded drug solution may be expected to saturate the "binding" sites on the glass or plastic surfaces, thus potentially mitigating the problem in subsequent solution transfers.

In the case of practically insoluble drugs, when solid is separated from slurry, the resulting "just-saturated" sample solution can decrease in concentration with each subsequent transfer, as surface adsorption removes some of the compound from solution, taking the system further away from the "just-saturated" state, since there is no solid to re-equilibrate the perturbed solution. In filtration, adsorption to filters is well recognized problem [117]. However, centrifugation as a method for separating solid from saturated solution is not free from similar adsorption effects. Consider the Bergström et al. [118] study where centrifugation was done twice: "The excess solid was separated from the solution by centrifugation at $23000 \mathrm{~g}$ for $15 \mathrm{~min}$. After the centrifugation, approximately $0.25 \mathrm{~mL}$ of the supernatant was sampled with Pasteur glass pipettes and dispensed into glass insert vials. These vials were placed in Eppendorf tubes and centrifuged for 15 min a second time to obtain complete separation of the solution and the remaining solid. After the centrifugation, the supernatant was withdrawn with Pasteur pipettes and dispensed into glass HPLC vials. Glass was used throughout this procedure to minimize the risk of underestimating the solubility owing to adsorption of the drugs to plastics." It was not explicitly reported that the Pasteur glass pipettes and HPLC vials were pre-treated with sample before use. Table 2 lists the intrinsic solubility values of twelve practically-insoluble drugs taken from the above study, and compares them to corresponding averaged intrinsic solubilities taken from other published studies (with all values normalized to $25^{\circ} \mathrm{C}$ [26]).

Figure 19 is a plot of the above comparison. Notably, the $\log S_{0}$ values from [118] are 1-3 (or more) log units lower than the average values reported from other published studies, with the exception of that of itraconazole. Although there may be a number of plausible explanations for the differences, it might be useful to consider the possibility that the double-centrifugation step led to under-estimated solubility, due to adsorption to the glass surfaces of the transfer vials. In the case of itraconazole, the value reported by Glomme et al. [117] may have had more adsorption than even in [118]. The former group used plastic UniPrep filter vials: "Even with the UniPrep filters, a loss of drug can sometimes occur through adsorption on the filter membrane. For that reason, the first drops of the filtrate should be discarded."

\section{Combining Centrifugation with Filtration}

It is potentially a faulty procedure, when separating solid from solution: to centrifuge the suspension first and then subject the centrifugate to a further filtration step. The reason is very simple: once the solid is removed, a "just-saturated" solution has no capacity to re-establish equilibrium when exposed to the surface of a filter, where adsorption usually takes place to some extent, and can account for a large fraction of the mass of a practically-insoluble compound. 
Table 2. Potential sample loss due to adsorption in multiple transfers

\begin{tabular}{cccccc}
\hline Drug & $\log {S_{0}}^{{ }^{2}}$ & SD & $\log S_{0}{ }^{b}$ & SD $^{\mathbf{c}}$ & Lit. Refs. \\
\hline Albendazole & -6.01 & 0.05 & -5.65 & 0.15 & {$[119,120]$} \\
Carvedilol & -6.15 & 0.03 & -5.32 & 0.50 & {$[48,121-124]$} \\
Cinnarizine & -7.73 & 0.04 & -7.14 & 0.28 & {$[27,125,126]$} \\
Danazol & -7.44 & 0.34 & -6.02 & 0.28 & {$[117,125,127-130]$} \\
Felodipine & -6.56 & 0.03 & -5.77 & 0.10 & {$[117,131,132]$} \\
Glimepiride & -7.90 & 0.10 & -6.85 & 0.36 & {$[133-135]$} \\
Itraconazole & -8.48 & 0.61 & -9.67 & $--{ }^{d}$ & {$[117]$} \\
Rimonabant & -7.02 & 0.06 & -6.35 & $--{ }^{d}$ & {$[136]$} \\
Tamoxifen & -8.54 & 0.15 & -7.54 & 0.72 & {$[137-140]$} \\
Terfenadine & -7.94 & 0.27 & -6.99 & 1.05 & {$[2,21,110,141-147]$} \\
Tolfenamic Acid & -7.87 & 0.05 & -6.16 & $-{ }^{d}$ & {$[137]$} \\
Troglitazone & -7.80 & 0.43 & -5.34 & 0.78 & {$[148,149]$}
\end{tabular}

\footnotetext{
a Solid separated from slurry by two centrifugations [118].

${ }^{\mathrm{b}}$ Averaged for the corresponding drugs from other published sources; values transformed to $25{ }^{\circ} \mathrm{C}$ before averaging.

${ }^{c}$ Interlaboratory standard deviations, with references in the last column. ${ }^{d}$ Single source comparison.
}

\section{Filtration}

Solubility suspensions which fail to clarify or sediment, must be filtered through membrane or glass filter. Filtration should not be done right after stirring, since the formation of a supersaturated solution upon intensive agitation may lead to an overestimated solubility. Since majority of compounds are inclined to supersaturation, it is recommended to allow the sample standing after stirring (as done in the sedimentation technique) to avoid this problem. Another source of error using filtration is the possible adsorption of the solid to the filter material, as just described. This can be substantially reduced or eliminated by pre-saturating the filter and discarding the initial portions of filtrate. Less attention was devoted in the literature to the proper filter type selection. A recent study has revealed the significant role of hydrophilic or hydrophobic type of filters on the solubility results of ionizable molecules [150]. The selection of appropriate filter type requires some knowledge of the acid-base chemistry of the sample. For the filtration of the ionized form of the compound, hydrophobic filter can be recommended. For unionized drugs, the uncharged-form hydrophilic filter can be recommended. These suggestions can be supported by the solubility results of papaverine $\left(\mathrm{p} K_{\mathrm{a}}\right.$ 6.39) measured at three $\mathrm{pH}$ values in the Britton-Robinson (BR) buffer, using different type of filters (Table 3 ).

A further aspect in the selection of the filter may be its diameter and pore size. The most frequently used ideal membrane filter parameters are $25 \mathrm{~mm}$ diameter and $0.22 \mu \mathrm{m}$ or $0.45 \mu \mathrm{m}$ pore size. These pore sizes are suitable for macrocrystals and micronized particles, however nano-size solids require specific filters. The solution should be slowly filtered in small 300-600 $\mu \mathrm{L}$ portions. The first 1-5 aliquots saturate the filter, and thus need to be discarded, while the sixth aliquot can be used for concentration measurement. 


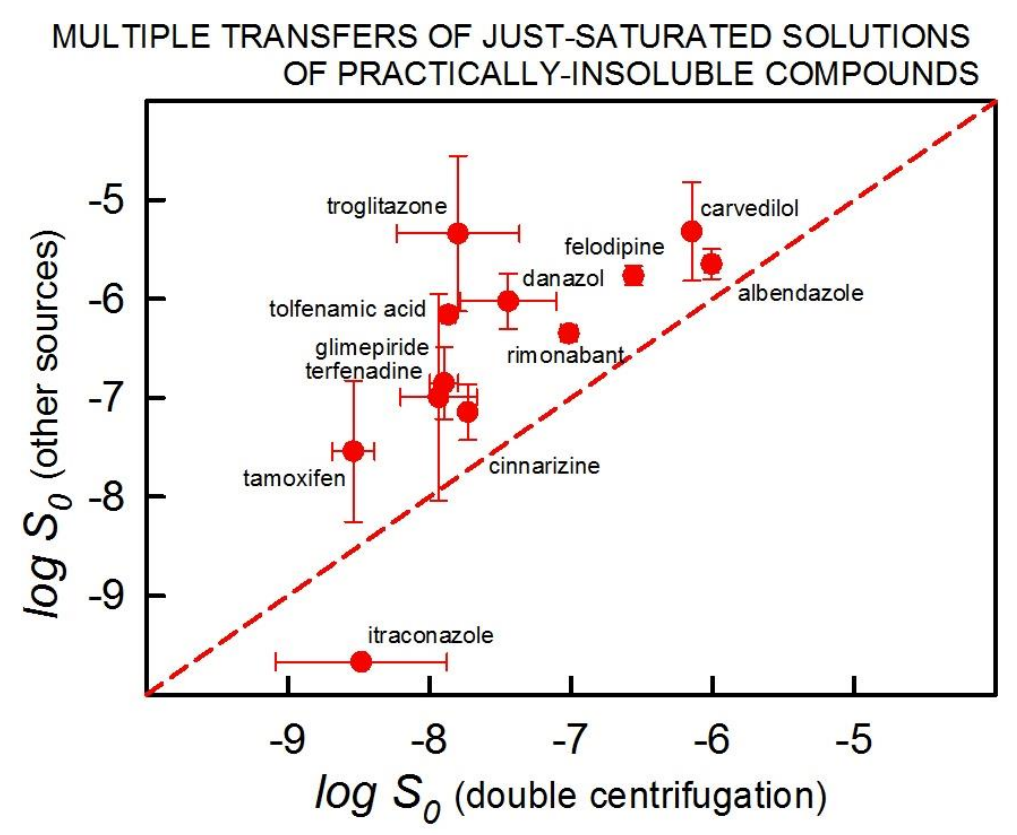

Figure 19. The comparison of the intrinsic solubility values of twelve practically-insoluble drugs [118] to the corresponding averaged values taken from other published studies (with values transformed to $25^{\circ} \mathrm{C}$ [26]). Notably, the $\log S_{0}$ values along the horizontal axis are 1-3 (or more) log units lower than the average values reported from other published, with the exception of that of itraconazole. See text.

Table 3. Effect of separation method and filter type on the equilibrium solubility of papaverine

\section{Method of separation of}

solid from the saturated

solution
pH 2.80

$100 \%$ charged form

\section{$S_{\mathrm{pH}}(\mathrm{mg} / \mathrm{mL})$}

pH 6.40

$50 \%$ charged

$50 \%$ uncharged form
pH 9.55
$100 \%$ uncharged form
Sedimentation

Filtration on hydrophilic

filter (PVDF)

Filtration on

hydrophobic filter

(nylon)
39.1

36.3

36.6
20.4

0.017

22.4

0.016

9.52

0.011 


\section{Recommendations for phase separation}

Sedimentation is recommended as the safest method for separation of the solid from the saturated solution. For non-clarifying, opalescent colloid solutions the centrifugation can be used.

If filtration cannot be avoided, then it is essential that the proper filter type is selected. For polar, ionized species hydrophobic (nylon) is recommended, while for unionized species the hydrophilic type filters (PVDF, PES) are recommended. The filtration should be done after sedimentation (resting time), and not directly after agitation. Pre-saturation of the filter is necessary. The initial portions of filtrate should be discarded.

\section{Solubility Units, Conversion Issues, Tabulation of Results and the Use of Logarithmic Plots}

Solubility measurements have been reported in many different concentration units: weight/volume (e.g., $\mathrm{mg} / \mathrm{mL}, \mu \mathrm{g} / \mathrm{mL}$ ), $\mathrm{mol} / \mathrm{L}$ (molarity, $\mathrm{M}$ ), $\mathrm{mol} / \mathrm{kg}$ (molality, $\mathrm{m}$ ), mole fraction, and mass fraction - just to name a few [4]. Mole fraction, mass fraction, and molality units are popular choices when solubility is determined over a wide range of temperatures, since the units do not depend on the density of the solutions in aqueous solutions. Also, such units are convenient to use with viscous solutions [151]. When solubility is reported in "practical" $\mathrm{mg} / \mathrm{mL}$ or $\mu \mathrm{g} / \mathrm{mL}$ units, the equivalent molecular weight needs to be clearly indicated (e.g., "concentration is expressed as free base equivalents," meaning that it is the molecular weight of the free base that is to be used to convert practical units to molarity units).

As different units are in common usage, it is too easy to make a mistake in converting the units to the common molarity scale. Solubility should be presented in log units (preferably based on molarity), since (a) direct values span over many orders of magnitude and cannot be accurately depicted in S-pH plots at the low end of the scale, and (b) since errors in log values do not depend on the magnitude of the log solubility. When molality units are used, it would be useful if the actual solution density is reported at the various temperatures studied.

\section{Recommendation for Reporting Solubility Units}

It is recommended that solubility be tabulated both in molarity and in practical $(\mathrm{mg} / \mathrm{mL})$ units, as done in the Handbook of Aqueous Solubility Data (Yalkowsky et al. [152]). Standard deviations in the measured solubility (based on averaging three or more values) should be included in the table of values. Additionally, a graphical display of logS vs. pH (but not $S$ vs. $\mathrm{pH}$ ) would be helpful.

\section{Conclusions}

This commentary drew on the extensive experimental knowledge and experiences of several laboratories with which the authors are associated. Reviewed were a number of factors that can affect the quality of equilibrium solubility measurement as a function of $\mathrm{pH}$ of druglike molecules, especially those which are only sparingly soluble. It was concluded that the traditional shake-flask and the potentiometric CheqSol methods 
could be used, provided that proper assay protocol is followed. It was stressed that independently-determined $\mathrm{p} K_{a}$ values of the drug be used in the analysis of the logS- $\mathrm{pH}$ data. The importance of solid state characterization was also stressed, citing several case studies of polymorphic and cocrystal transformations. The complexity on the solution side of solubility-pH measurement was illustrated with several case studies, where aggregates (micellar and sub-micellar) and drug-buffer or drug-coformer complexes appeared to form. The importance of measuring $\mathrm{pH}$ accurately in buffered and unbuffered solutions was discussed at length. Methods and pitfalls of separating solid from saturated solutions were critically discussed. The proper reporting of the temperature, ionic strength, buffer capacity, and other experimental detail was encouraged. When such "good practices" could be followed, it is expected that high quality results in solubility measurement could be achieved.

\section{Glossary}

API

Bjerrum plot

$\mathrm{BR}$

CCDC

CheqSol

$\mathrm{CMC}$

CSC

CSD

CV

DTT

DSC

DASH

GSAS

$\mathrm{HH}$

I

$\mathrm{IR}$

$K_{\mathrm{n}}$

$K_{\mathrm{sp}}$

LP

LLPS

MUB

MSZ active pharmaceutical ingredient

Average number of ionizable protons, $\bar{n}_{H}$, bound to a weak acid/base, plotted as a function of $\mathrm{pH}$. For example, for a monoprotic acidic drug with a $\mathrm{pK}_{\mathrm{a}} 4.5, \overline{\mathrm{n}}_{\mathrm{H}}=1.0$ at $\mathrm{pH} 2, \overline{\mathrm{n}}_{\mathrm{H}}=0.5$ at $\mathrm{pH} 4.5$, and $\overline{\mathrm{n}}_{\mathrm{H}}=0.0$ at $\mathrm{pH} 9[27,153]$.

Britton-Robinson buffer [154]

Cambridge Crystallographic Data Centre

Chasing Equilibrium Solubility

critical micelle concentration

critical stabilization concentration

Cambridge Structural Database

coefficient of variation

Dissolution Titration Template (potentiometric method to determine intrinsic solubility, $\mathrm{S}_{0}$ )

differential scanning calorimetry

Interactive package from the Cambridge Crystallographic Data Centre (CCDC) for solving crystal structures from powder diffraction data (https://www.ccdc.cam.ac.uk/solutions/csdmaterials/components/dash/)

General Structure Analysis System. Comprehensive system created by Allen C. Larson and Robert B. Von Dreele of Los Alamos National Laboratory for the refinement of structural models (http://www.ncnr.nist.gov/xtal/software/downloads.html), for both X-ray and neutron diffraction data

Henderson-Hasselbalch equation (e.g., Eq. 1)

ionic strength of the solution

infrared spectroscopy

aggregation constant, where $\mathrm{n}$ is the degree of aggregation

drug-salt or drug-coformer (cocrystal) solubility product

liquid junction potential

liquid-liquid phase separation

Minimalist Universal Buffer

Metastable Zone 
$[P C]^{2} \quad$ Potentiometric Cycling for Polymorph Creation

$\mathrm{p} K_{\mathrm{a}} \quad$ negative logarithm of the ionization constant

$\mathrm{pK}_{\mathrm{a}}{ }^{\text {Gibbs }} \quad \mathrm{pH}$ where the drug in the uncharged form co-precipitates with the drug in the salt form

$\mathrm{pH}_{\text {sat }} \quad$ equilibrium $\mathrm{pH}$ of a saturated solution

PXRD powder X-ray diffraction characterization of the solid form

$S \quad$ solubility, ideally expressed in units of $\mathrm{mol} / \mathrm{L}(\mathrm{M}), \mu \mathrm{g} / \mathrm{mL}$, or $\mathrm{mg} / \mathrm{mL}$

$S_{0} \quad$ "intrinsic" solubility (i.e., the solubility of the uncharged form of the compound)

$S_{\mathrm{w}} \quad$ "water" solubility, defined by dissolving enough pure free acid/base in distilled water (or water containing an inert salt - as ionic strength adjustor) to form a saturated solution. The final $\mathrm{pH}$ of the suspension, $\mathrm{pH}_{\text {sat }}$, and $S_{0}$ can be calculated by the $\mathrm{HH}$ equation (when valid), provided the true $\mathrm{p} K_{\mathrm{a}}$ is known. Compound added as a salt form may disproportionate into free acid/base, depending on how much solid had been added. It is not generally possible to calculate the $\mathrm{pH}$ and $S_{0}$ of such a drug salt suspension.

$S_{\mathrm{pH}} \quad$ "pH buffer" solubility (i.e., the total solubility of the compound at a well-defined $\mathrm{pH}_{\text {sat }}$ )

TGA thermogravimetric analysis

Acknowledgements: T.V. wishes to thank Drs. Gordana Popović and Lidija Pfendt for many helpful discussions. A.A. wishes to thank Drs. Agustin Asuero (Seville Univ.), Michael Abraham (Univ. College London), and Lennart Lindfors of AstraZeneca (Mölndal) for stimulating discussions. 


\section{References}

[1] A. Llinàs, R.C. Glen, J.M. Goodman. J. Chem. Inf. Model. 48 (2008) 1289-1303.

[2] A.J. Hopfinger, E.X. Esposito, A. Llinàs, R.C. Glen, J.M. Goodman. J. Chem. Inf. Model. 49 (2009) 1-5.

[3] E. Baka, J. E.A. Comer, K. Takács-Novák. J. Pharm. Biomed. Anal. 46 (2008) 335-341.

[4] A. Avdeef. ADMET \& DMPK 3 (2015) 84-109.

[5] N.K. Pandit, J.M. Strykowski. J. Pharm. Sci. 78 (1989) 767-770.

[6] N.K. Pandit, J.M. Strykowski, L. Shtohryn. Int. J. Pharm. 50 (1989) 7-13.

[7] T. Higuchi, A.K Connors. Adv. Anal. Chem. Instrum. 4 (1965) 117-212.

[8] D.J.W. Grant, T. Higuchi. Solubility Behavior of Organic Compounds, John Wiley \& Sons, New York, 1990.

[9] J.T. Carstensen. Advanced Pharmaceutical Solids, Marcel Dekker, Inc., New York, 2001.

[10] M. Pudipeddi, A.T.M. Serajuddin, D.J.W. Grant, P.H. Stahl. Solubility and dissolution of weak acids, bases, and salts. In: P.H. Stahl, C.G. Wermuth (Eds.). Handbook of Pharmaceutical Salts: Properties, Selection, and Use, Wiley-VCH, Weinheim, 2002, pp. 19-39.

[11] B.D. Anderson, K.P. Flora. Preparation of water-soluble compounds through salt formation. In: Wermuth, C.G. (Ed.), The Practice of Medicinal Chemistry. Academic Press, London, 1996, pp. 739-754.

[12] W.Q. Tong, G. Whitesell. Pharm. Dev. Technol. 3 (1998) 215-223.

[13] A.T.M., Serajuddin, M. Pudipeddi. Salt selection strategies. In: P.H. Stahl, C.G. Wermuth (Eds.), Handbook of Pharmaceutical Salts: Properties, Selection, and Use, Wiley-VCH, Weinheim, 2002, pp. 135-160.

[14] A.T. Serajuddin. Adv. Drug Deliv. Rev. 59 (2007) 603-613.

[15] A.F. Casares, W.M. Nap, G.T. Figás, P. Huizenga, R. Groot, M. Hoffmann. An evaluation of salt screening methodologies. J. Pharm. Pharmacol. 67 (2015) 812-822.

[16] P.H. Stahl, C.G. Wermuth (Eds.). Handbook of Pharmaceutical Salts Properties, Selection, and Use. John Wiley \& Sons, New York, 2008, 374 pp.

[17] H.G. Brittain. J. Pharm. Sci. 86 (1997) 405-411.

[18] M. Pudipeddi, A.T.M. Serajuddin. J. Pharm. Sci. 94 (2005) 929-939.

[19] P.H. Stahl. Salt selection. In: R. Hilfiker (Ed.). Polymorphism in Pharmaceutical Industry, Wiley-VCH, Weinheim, 2006, pp. 309-322.

[20] J. Bernstein. Polymorphism of Molecular Crystals. 2007, Oxford University Press, Oxford, 428 pp.

[21] A. Avdeef. Adv. Drug Deliv. Rev. 59 (2007) 568-590.

[22] G. Völgyi, A. Marosi, K. Takács-Novák, A. Avdeef. ADMET \& DMPK 1 (2013) 48-62.

[23] A. Avdeef. ADMET \& DMPK 2 (2014) 33-42.

[24] A. Avdeef. ADMET \& DMPK 2 (2014) 43-55.

[25] G. Butcher, J. Comer, A. Avdeef. ADMET \& DMPK 3 (2015) 131-140.

[26] A. Avdeef. ADMET \& DMPK 3 (2015) 298-344.

[27] A. Avdeef. Absorption and Drug Development Second Edition. Wiley-Interscience: New York. 2012, pp. 251-318.

[28] S.J. Nehm, B. Rodriguez-Spong, N. Rodriguez-Hornedo. Cryst. Growth Des. 6 (2006) 592-600.

[29] L.S. Reddy, S. Bethune, A. Jayasankar, N. Rodriguez-Hornedo. Cryst. Growth Des. 9 (2009) 378-385.

[30] D. Good, N. Rodríguez-Hornedo. Cryst. Growth Des. 9 (2009) 2252-2264.

[31] S. Bethune, N.C. Huang, A. Jayasankar, N. Rodríguez-Hornedo. Cryst. Growth Des. 9 (2009) 976-3988.

[32] D. Good, N. Rodriguez-Hornedo. Cryst. Growth Des. 10 (2010) 1028-1032.

[33] N. Huang, N. Rodríguez-Hornedo. Cryst. Growth Des. 10 (2010) 2050-2053. 
[34] A. Alhalaweh, L. Roy, N. Rodríguez-Hornedo. S.P. Velaga. Molec. Pharmaceut. 9 (2012) 2605-2612.

[35] C. Maheshwari, V. André, S. Reddy, L. Roy, T. Duarte, N. Rodríguez-Hornedo. Cryst. Eng. Comm. 14 (2012) 4801-4811.

[36] A. Alhalaweh, A. Sokolowski, N. Rodríguez-Hornedo, S.P. VelagaCryst. Growth Des. 11 (2011) 3923-3929.

[37] J. Wouters, L. Quere (Eds.), Pharmaceutical Salts and Co-crystals, RSC Publishing, London, 2011, 391 pp.

[38] D. Murphy, F. Rodríguez-Cintrón, B. Langevin, R. C. Kelly, N. Rodríguez-Hornedo. Int. J. Pharm. 246 (2002) 121-134.

[39] J.P. Boetkera, J. Rantanena, L. Arnfasta, M. Doretha, D. Raijadaa, K. Loebmanna, C. Madsena, J. Khanb, T. Radesa, A. Müllertza, A. Hawleyd, D. Thomase, B.J. Boyd. Eur. J. Pharm. Biopharm. 100 (2016) 119-127.

[40] H.N. Po, N.M. Senozan. J. Chem. Educ. 78 (2001) 1499-1503.

[41] G. Völgyi, E. Baka, K.J. Box, J.E.A. Comer, K. Takács-Novák. Anal. Chim. Acta 673 (2010) 40-46.

[42] A. Avdeef. Pharm. Pharmacol. Commun. 4 (1998) 165-178.

[43] W.H. Streng. Int. J. Pharm. 186 (1999) 137-140.

[44] Z. Wang, L.S. Burrell, W.J. Lambert. J. Pharm. Sci. 91 (2002) 1445-1455.

[45] A. Avdeef, J.J. Bucher. Anal. Chem. 50 (1978) 2137-2142.

[46] A. Avdeef, C.M. Berger, C. Brownell. Pharm. Res. 17 (2000) 85-89.

[47] J.C. Machado, A.D. Lange, V. Todeschini, N.M. Volpato. AAPS PharmSciTech 15 (2014) 189-197.

[48] D. Schönherr, U. Wollatz, D. Haznar-Garbacz, U. Hanke, K.J. Box, R. Taylor, R. Ruiz, S. Beato, D. Becker, W. Weitschies. Eur. J. Pharm. Biopharm. 92 (2015) 155-170.

[49] H.A. Krebs, J.C. Speakman. J. Chem. Soc. (1945) 593-595.

[50] I. Zimmermann. Int. J. Pharm. 13 (1983) 57-65.

[51] D.L. Ross, C.M. Riley. Int. J. Pharm. 63 (1990) 237-250.

[52] A. Tsuji, E. Nakashima, S. Hamano, T. Yamana. J. Pharm. Sci. 67 (1978) 1059-1066.

[53] E. Shoghi, E. Fuguet, E. Bosch, C. Ràfols. Eur. J. Pharm. Sci. 48 (2012) 290-300.

[54] T.M. Ward, J.B. Weber. J. Agr. Food Chem. 16 (1968) 959-961.

[55] J.B. Weber. Spectrochim. Acta 23 (1967) 458-461.

[56] S. Li, S. Wong, S. Sethia, H. Almoazen, Y.M. Joshi, A.T.M. Serajuddin. Pharm. Res. 22 (2005) 628-635.

[57] S. Li, P. Doyle, S. Metz, A.E. Royce, A.T.M. Serajuddin J. Pharm. Sci. 94 (2005) 2224-2231.

[58] G. Zografi, I. Zarenda. Biochem. Pharmacol. 15 (1966) 591-598.

[59] A.S. Indulkar, K.J. Box, R. Taylor, R. Ruiz, L.S. Taylor. Mol. Pharmaceut. 12 (2015), 2365-2377.

[60] A. Avdeef, O. Tsinman. Pharm. Res. 25 (2008) 2613-2627.

[61] C. Dagenais, A. Avdeef, O. Tsinman, A. Dudley, R. Beliveau. Eur. J. Pharm. Sci. 38 (2009) 121-137.

[62] S.-T. Liu, A. Hurwitz. J. Colloid Int. Sci. 60 (1977) 410-413.

[63] K. Takács-Novák, G. Völgyi. Best practice for saturation shake-flask measurements of thermodynamic solubility. Abstracts - 4th World Conf. Phys.-Chem. Meth. Drug Disc. Dev., Red Island, Croatia, 21-24 Sep 2015.

[64] M. Stuart, K. Box. Anal. Chem. 77 (2005) 983-990.

[65] K.J. Box, G. Völgyi, E. Baka, M. Stuart, K. Takács-Novák, J.E. Comer. J. Pharm. Sci. 95 (6) (2006) 12981307.

[66] A. Llinàs, J.C. Burley, K.J. Box, R.C. Glen, J.M. Goodman. J. Med. Chem. 50 (2007) 979-983.

[67] M. Yasuda. Bull. Chem. Soc. Jpn. 32 (1959) 429-432.

[68] T. Shedlovsky. Electrolytes, Proc. Intern. Symp., Trieste, Italy, 1962, 1959, 146-151. 
[69] T. Shedlovsky. In Pesce, B. In Electrolytes. Ed.; Pergamon Press: New York, 1962; pp. 146-151.

[70] D. Kovala-Demertzi, D. Mentzafos, A. Terzis. Polyhedron 12 (1993) 1361-1370.

[71] A. Llinàs, J.M. Goodman. Drug Discov. Today 13 (2008) 198-210.

[72] A. Llinàs, L. Fábián, J.C. Burley, J. van de Streek, J.M. Goodman. Acta Cryst. E62 (2006) o4196-04199

[73] A. Llinàs, K.J. Box, J. C. Burley, R. C. Glen, J. M. Goodman. J. Appl. Cryst. 40 (2007) 379-381.

[74] A. F. Fioritto, S.N. Bhattachar, J.A. Wesley. Int. J. Pharm. 330 (2007) 105-113.

[75] C.H. Koo, S.H. Kim, W. Shin. Bull. Korean Chem. Soc. 6 (1985) 222-224.

[76] W. I. David, K. Shankland, N. Shankland. Chem. Commun. 8 (1998) 931-932.

[77] A. C. Larson, R. B. Von Dreele, R. B. General Structure Analysis System (GSAS), Report LAUR 86-748. LoS Alamos National Laboratory, New Mexico, USA, 2000.

[78] http://www.panalytical.com/XPert3-Powder.htm. Accession date: June 23, 2016.

[79] https://www.bruker.com/products/x-ray-diffraction-and-elemental-analysis/x-ray-diffraction/d8endeavor/overview.html. Accession date: June 23, 2016.

[80] T.N. Drebushchak, Yu.A. Chesalov, E.V. Boldyreva. Acta Crystallogr. B 65 (2009) 770-781.

[81] http://www.renishaw.com/en/invia-confocal-raman-microscope--6260. Accession date: June 23, 2016.

[82] S. Childs, N. Rodriguez-Hornedo, L.S. Reddy, A. Jayasankar, C. Maheshwari, L. McCausland, R. Shipplett, B. C. Stahly. Cryst. Eng. Comm. 10 (2008) 856-864.

[83] E.V. Boldyreva, S.G. Arkhipov, T.N. Drebushchak, V.A. Drebushchak, E.A. Losev, A.A. Matvienko, V.S. Minkov, D.A. Rychkov, Yu. V. Seryotkin, J. Stare, B.A. Zakharov. Chem. Eur. J. 21 (2015) 15395-15404.

[84] Y. Sakata. Agric. Biol. Chem. 25 (1964) 829-834.

[85] Y. Sakata. Agric. Biol. Chem. 25 (1964) 835-837.

[86] C.-Y. Lee, J.-T. Chen, W.-T. Chang, I.-M. Shiah. Fluid Phase Equilib. 343 (2013) 30-35.

[87] N. Schultheiss, A. Newman. Cryst. Growth Des. 9 (2009) 2950-2967.

[88] N. Shan, M.J. Zaworotko. Drug Discov. Today 13 (2008) 440-446.

[89] W. Jones, W.D.S. Motherwell, A.V. Trask. MRS Bull. 31 (2006) 875-879.

[90] A.V. Trask. Molec. Pharmaceut. 4 (2007) 301-309.

[91] N. Qiao, M. Li, W. Schlindwein, N. Malek, A. Davies, G. Trappitt. Int. J. Pharm. 419 (2011) 1-11.

[92] R. Thakuria, A. Delori, W. Jones, M.P. Lipert, L. Roy, N. Rodríguez-Hornedo. Int. J. Pharm. 453 (2013) 101125.

[93] H.G. Brittain. J. Pharm. Sci. 102 (2013) 311-317.

[94] Ö. Almarsson, M.L. Peterson, M. Zaworotko. Pharm. Patent Analyst 1 (2012) 313-327.

[95] R. Chadha, A. Saini, P. Arora, S. Bhandari. Crit. Rev. Ther. Drug Carrier Syst. 29 (2012) 183-218.

[96] F. Cao, G.L. Amidon, N. Rodriguez-Hornedo, G. E. Amidon. Molec. Pharmaceut. 13 (2016) 1030-1046.

[97] C. Maheshwari, A. Jayasankar, N.A. Khan, G.E. Amidon, N. Rodríguez-Hornedo. Cryst. Eng. Comm. 11 (2009) 493-500.

[98] D. Good, C. Miranda, N. Rodríguez-Hornedo. Cryst. Eng. Comm. 13 (2011) 1181-1189.

[99] N.J. Babu, A. Nangia. Cryst. Growth Des. 11 (2011) 2662-2679.

[100] D.R. Weyna, T. Shattock, P. Vishweshwar, M.J. Zaworotko. Cryst. Growth Des. 9 (2009) 1106-1123.

[101] I. Halasz, A. Puškarić, S.A.J. Kimber, P.J. Beldon, A.M. Belenguer, F. Adams, V. Honkimäki, R.E. Dinnebier, B. Patel, W. Jones, V. Štrukil, T. Friščić. Angew. Chemie Int. Ed. 52 (2013) 11538-11541.

[102] S.A. Myz, T.P. Shakhtshneider, K. Fucke, A.P. Fedotov, E.V. Boldyreva, V.V. Boldyrev, N.I. Kuleshova. Mendeleev Comm. 19 (2009) 272-274. 
[103] T. Friščić, W. Jones. Cryst. Growth Des. 9 (2009) 1621-1637.

[104] D. Braga, L. Maini, F. Grepioni. Chem. Soc. Rev. 42 (2013) 7638-7648.

[105] C.W. Pouton. Adv. Drug Deliv. Rev. 25 (1997) 47-58.

[106] V.P. Torchilin. J. Control. Rel. 73 (2001) 137-172.

[107] N. Huang, N. Rodríguez-Hornedo. J. Pharm. Sci. 100 (2011) 5219-5234.

[108] N. Rodriguez-Hornedo. S.J. Nehm, K.F. Seefeldt, Y. Pagan-Torres, C.J. Falkiewicz. Molec. Pharmaceut. 3 (2006) 362-367.

[109] M.H. Abraham, J. Le. J. Pharm. Sci. 88 (1999) 868-880.

[110] E. Rytting, K.A. Lentz, X.Q. Chen, F. Qian, S. Venkatesh. Pharm Res. 21 (2004) 237-244.

[111] G. Biedermann, L.G. Sillén. Arkiv. Kemi. 5 (1952) 425-440.

[112] A. Avdeef, J.E.A. Comer, S.J. Thomson. Anal. Chem. 65 (1993) 42-49.

[113] A. Avdeef, K.J. Box, J.E.A. Comer, M. Gilges, M. Hadley, C. Hibbert, W. Patterson, K.Y. Tam. J. Pharm. Biomed. Anal. 20 (1999) 631-641.

[114] V.S. Živanović, M.P. Pešić, V. Horváth, J. Madarász, I.N. Cvijetić, G.V. Popović, T.Ž. Verbić, A. Avdeef. Terfenadine solubility studies. 4th World Conference on Physico-Chemical Methods in Drug Discovery and Development, September 21-24, 2015, Red Island, Croatia, Abstracts P12.

[115] T. Higuchi, F.-M. Shih, T. Kimura, J.H. Rytting. J. Pharm. Sci. 68 (1979) 1267-1272.

[116] J.J. Palmgrén, J. Mönkkönen, T. Korjamo, A. Hassinen, S. Auriola. Eur. J. Pharm. Biopharm. 64 (2006) 369-378.

[117] A. Glomme, J. März, J.B. Dressman. J. Pharm. Sci. 94 (2005) 1-16.

[118] C.A.S. Bergström, C.M. Wassvik, K. Johansson, I. Hubatsch. J. Med. Chem. 50 (2007) 5858-5862.

[119] A. Avdeef, M. Kansy, S. Bendels, K. Tsinman. Eur. J. Pharm. Sci. 33 (2008) 29-41.

[120] B.I. Escher, C. Berger, N. Bramaz, J.H. Kwon, M. Richter, O. Tsinman, A. Avdeef. Environ. Tox. Chem. 27 (2008) 909-918.

[121] C.A.S. Bergström, K. Luthman, P. Artursson. Eur. J. Pharm. Sci. 22 (2004) 387-398.

[122] T. Loftsson, S.B. Vogensen, C. Desbos, P. Jansook. AAPS PharmSciTech 9 (2008) 425-430.

[123] K. Beattie, G. Phadke, J. Novakovic. Profiles Drug Subst. Excip. Rel. Method. 38 (2013) 113-157.

[124] Y.-L. Hsieh, G.A. Ilevbare, B. Van Eerdenbrugh, K.J. Box, M.V. Sanchez-Felix, L.S. Taylor. Pharm. Res. 29 (2012) 2738-2753.

[125] S. Clarysse, J. Brouwers, J. Tack, P. Annaert, P. Augustijns. Eur. J. Pharm. Sci. 43 (2011) 260-269.

[126] S.R. Johnson, X.Q. Chen, D. Murphy, O. Gudmundsson. Mol. Pharmaceut. 4 (2007) 513-523.

[127] A. Glomme, et al. In: B. Testa, et al. (eds), Pharmacokinetic Profiling in Drug Research. Wiley-VCH 2006, pp 259-280.

[128] V. Bakatselou, R.C. Oppenheim, J.B. Dressman. Pharm. Res. 8 (1991) 1461-1469.

[129] S.D. Mithani, R.C. Oppenheim, J.B. Dressman. Pharm. Res. 13 (1996) 163-167.

[130] L.-A. Erlich, D. Yu, D.A. Pallister, R. Saul Levinson, D.G. Gole, P.A. Wilkinson, R.E. Erlich, L.E. Reeve, T.X. Viegas. Int. J. Pharm. 179 (1999) 49-53.

[131] C.A.S. Bergström, C.M. Wassvik, U. Norinder, K. Luthman, P. Artursson. J. Chem. Inf. Comput. Sci. 44 (2004) 1477-1488.

[132] S.A. Raina, B. Van Eerdenbrugh, D.E. Alonzo, H. Mo, G.G.Z. Zhang, Y.Gao, L.S. Taylor. J. Pharm. Sci. 104 (2015), 1981-1992.

[133] T. Taupitz, J.B. Dressman, S. Klein. Eur. J. Pharm. Biopharm. 84 (2013) 208-218.

[134] L.Y. Narasimham, V.D. Barhate. J. Pharmacy Res. 4 (2011) 532-536. 
[135] S. Grbić, J. Parojčić, A. Malenović, Z. Djurić, M. Maksimović. J. Chem. Eng. Data 55 (2010) 1368-1371.

[136] J.H. Fagerberg, E. Karlsson, J. Ulander, G. Hanisch, C.A.S. Bergström. Pharm. Res. 32 (2014) 578-589.

[137] C.A.S. Bergström, M. Strafford, L. Lazorova, A. Avdeef, K. Luthman, P. Artursson. J. Med. Chem. 46 (2003) 558-570.

[138] C.M. Wassvik, A.G. Holmén, C.A.S. Bergström, I. Zamora, P. Artursson. Eur. J. Pharm. Sci. 29 (2006) 294305.

[139] J.H. Fagerberg, O. Tsinman, N. Sun, K. Tsinman, A. Avdeef, C.A.S. Bergström. Mol. Pharmaceut. 7 (2010) 1419-1430.

[140] G. Ottaviani, S. Wendelspiess, R. Alvarez-Sánchez. Mol. Pharmaceut. 12 (2015) 1171-1179.

[141] A. Avdeef, C.M. Berger. Eur. J. Pharm. Sci. 14 (2001) 281-291.

[142] K.J. Box, J.E.A. Comer. Curr. Drug Metab. 9 (2008) 869-878.

[143] C. Sköld, S. Winiwarter, J. Wernevik, F. Bergström, L. Engström, R. Allen, K. Box, J. Comer, J. Mole, A. Hallberg, H. Lennernäs, T. Lundstedt, A.-L. Ungell, A. Karlén. J. Med. Chem. 49 (2006) 6660-6671.

[144] W.H. Streng, S.K. Hsi, P.E. Helms, H.G.H. Tan. J. Pharm. Sci. 73 (1984) 1679-1684.

[145] B. Bard, S. Martel, P.-A. Carrupt. Eur. J. Pharm. Sci. 33 (2008) 230-240.

[146] S. Budavari, et al. The Merck Index. 12th Ed., Windholz M (Ed.) Rahway, NJ: Merck and Co., Inc., 1996.

[147] T. Verbić. Abstracts. 4th World Conf. on Phys.-Chem. Meth. Drug Disc. Dev. (PCMDDD-4). Red Island, Croatia. 21-24 Sep 2015.

[148] E. Nicolaides, E. Galia, C. Efthymiopoulos, J.B. Dressman, C. Reppas. Pharm. Res. 16 (1999) 1876-1882.

[149] N. Suzuki, K. Kasahara, H. Hasegawa, T. Kawasaki. Int. J. Pharm. 248 (2002) 71-80.

[150] G. Völgyi, K. Takács-Novák. Filtration as phase separation technique in saturation shake-flask solubility determination method. Abstracts - 4th World Conf. Phys.-Chem. Meth. Drug Disc. Dev., Red Island, Croatia, 21-24 Sep 2015.

[151] S. Singh, T. Parikh, H.K. Sandhu, N.H. Shah, A.W. Malick, D. Singhal, A.T.M. Serajuddin. Pharm. Res. 30 (2013) 1561-1573.

[152] S.H. Yalkowsky, Y. He, P. Jain. Handbook of Aqueous Solubility Data, Second Edition. CRC Press, Boca Raton, FL, 2010.

[153] H.T.S. Britton, R.A. Robinson. J. Chem. Soc. (1931) 1456-1462.

[154] J. Bjerrum. Metal-Ammine Formation in Aqueous Solution, Haase, Copenhagen, 1941.

[155] D.D. Perrin, B. Dempsey. Buffers for pH and Metal Ion Control, Chapman and Hall, London, 1974.

[156] United States Pharmacopeia-National Formulary (USP25 NF20), 2002.

[157] T.C. Mcllvaine. J. Biol. Chem. 49 (1921) 183-186.

[158] F.H. Sweeton, R.E. Mesmer, C.F. Baes, Jr. J. Solut. Chem. 3 (1974) 191-214.

[159] A. Avdeef. Anal. Chim. Acta 148 (1983) 237-244. 


\section{Appendix A - Buffer Capacity and lonic Strength - Too Little, Too Much, or Just About Right}

Many buffer formulations have been described in the literature. Very useful detailed descriptions of specialty buffers have been tabulated by Perrin and Dempsey [155]. In this Appendix, five examples of buffer solutions are described, with emphasis on use in solubility measurements.

\section{Unbuffered Water}

Figure A1a shows the buffer capacity and ionic strength distribution as a function of $\mathrm{pH}$ for the "blank" titration shown in Figure A2a.
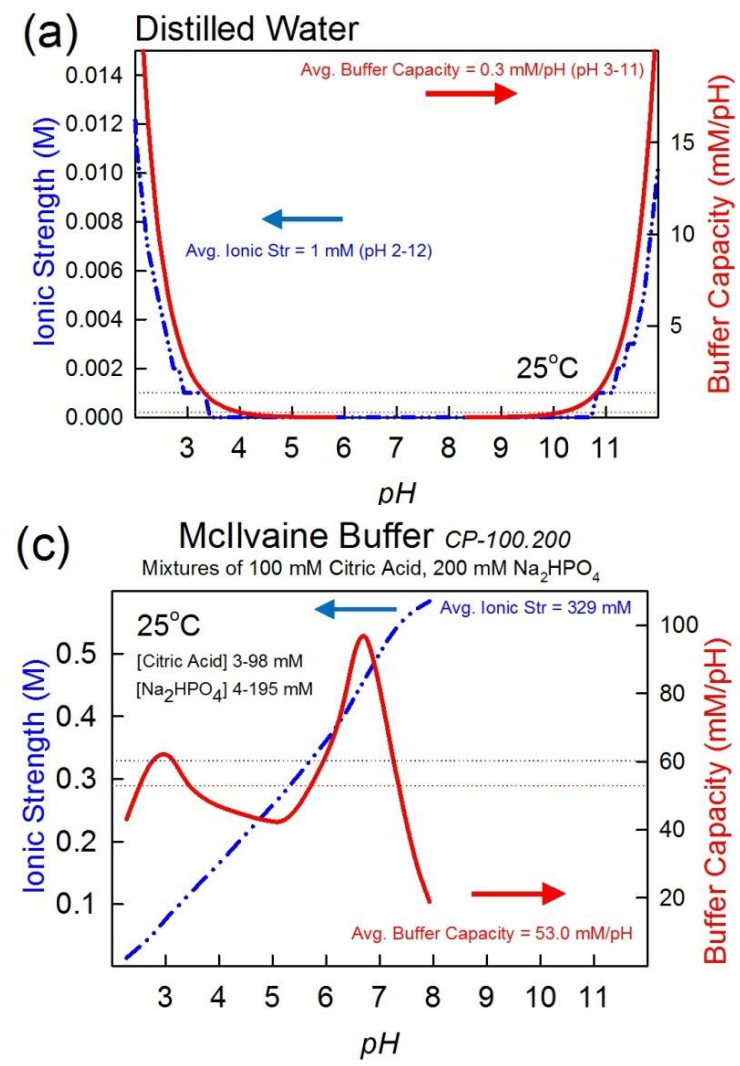

(e) Minimalist Solubility Buffer AEM-10.10.30
$10 \mathrm{mM}$ Acetic Acid, $10 \mathrm{mM}$ Ethylenediamine, $30 \mathrm{mM}$ Mesylic Acid

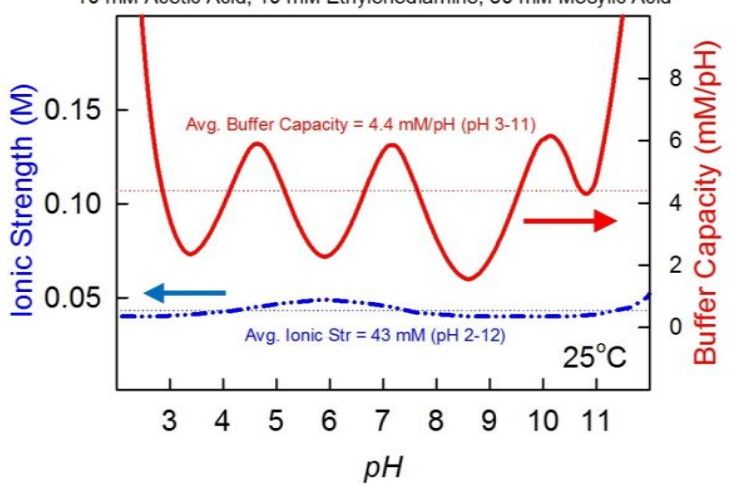

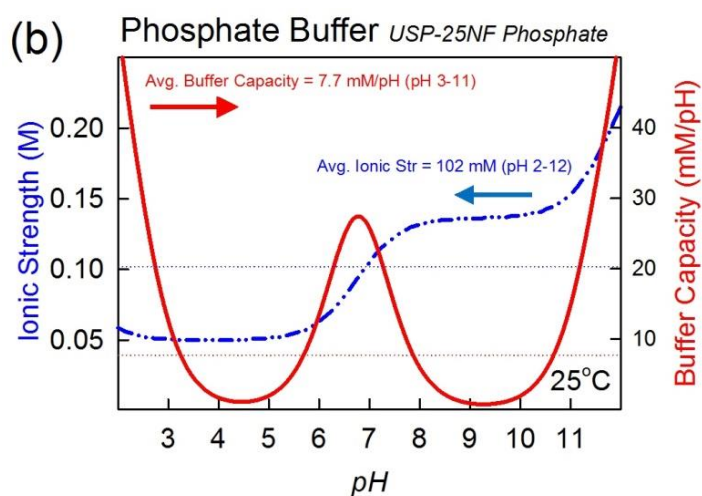

(d) Britton-Robinson Buffer $A P B-40.40 .40$

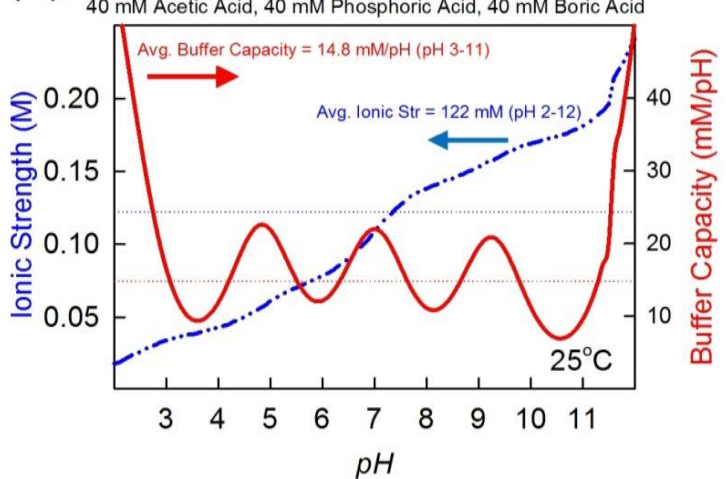

(f) Minimalist Solubility Buffer $A E T-25.25 .75$

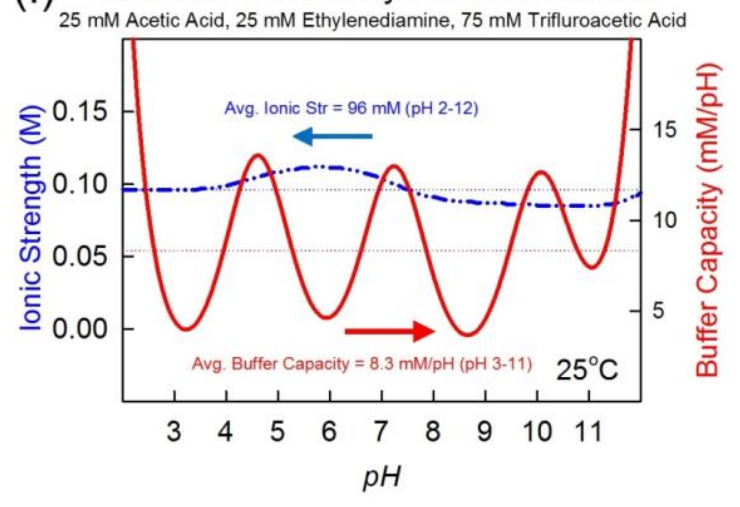

Figure A1. Plots of the buffer capacity and ionic strength as a function of $\mathrm{pH}$ for the unbuffered "blank" (a) solution, for the common phosphate buffer (b), and for four "universal" buffer solutions (c-f).

This is a case of the least buffered aqueous solution possible, where trace level of buffering is solely 
provided by the ionization of pure water. The region between $\mathrm{pH} 4$ and 10 is characterized by buffer capacity < $0.005 \mathrm{mM} / \mathrm{pH}$ and ionic strength $<0.0005 \mathrm{M}$. The commercial $\mathrm{p} K_{\mathrm{a}}$ analyzer dispensers adding minimum volumes of $0.5 \mathrm{M} \mathrm{HCl}$ or $\mathrm{NaOH}$ would not be able to resolve $\mathrm{pH}$ points any better than about $2 \mathrm{pH}$ units in the vicinity of $\mathrm{pH}$ 7. This case may be simply called the "unbuffered" solution.

\section{USP 50 mM Phosphate Buffer}

Figure A1b shows the buffer capacity and ionic strength distribution as a function of $\mathrm{pH}$ for the extended version of the popular $50 \mathrm{mM}$ phosphate buffer defined in U.S. Pharmacopeia - National Formulary No. 25 [156]. A characteristic feature is the high buffering occurring near $\mathrm{pH} 6.8(27.4 \mathrm{mM} / \mathrm{pH})$, as shown in the figure. Buffer capacity becomes minimal around $\mathrm{pH} 4.4(1.0 \mathrm{mM} / \mathrm{pH})$ and $9.3(0.7 \mathrm{mM} / \mathrm{pH})$. This is just about enough capacity for commercial $\mathrm{p} K_{\mathrm{a}}$ analyzers to adjust the $\mathrm{pH}$ of the phosphate solutions using typical titrants. Although the average ionic strength is near $0.1 \mathrm{M}$, the value increases from $0.05 \mathrm{M}$ to $0.14 \mathrm{M}$ as $\mathrm{pH}$ is adjusted across the $\mathrm{pH} 6.8$ region, as indicated in Figure A1b. Compensation for this level of change in ionic strength is theoretically feasible [22]. The phosphate buffer is suitable for most applications in solubility measurement. But one could do better.

\section{Mcllvaine Universal Buffer}

The Mcllvaine universal buffer [157] is sometimes used in dissolution studies as a function of $\mathrm{pH}$. It is formulated by adding different mixtures of $100 \mathrm{mM}$ citric acid (3-98 mM) and $200 \mathrm{mM} \mathrm{Na}{ }_{2} \mathrm{HPO}_{4}(4-195 \mathrm{mM})$, to cover the $\mathrm{pH}$ range from 2 to 8 . The buffer mixture has high buffer capacity, to be sure. Its unfavorable property vis-à-vis solubility measurement is that ionic strength varies from near zero to $0.5 \mathrm{M}$, as can be seen in Figure A1c. Also, the very high and continuously-variable phosphate concentration does not make this a suitable buffer for studying sparingly-soluble basic drugs, since analysis of drug-phosphate salt precipitates could be unwieldy [24]. In some cases the Mcllvaine buffer could be useful for studying acidic drugs. But there are better choices.

\section{Britton-Robinson Universal Buffer}

A very well formulated universal buffer is that of Britton-Robinson [153]. It consists of equimolar (e.g., $40 \mathrm{mM}$ ) mixtures of acetic acid, phosphoric acid, and boric acid [22, 45] Since the initial pH about 1.9, adjustment of the buffer with $\mathrm{NaOH}$ titrant can set the $\mathrm{pH}$ over a wide range. As Figure A1d shows, the buffer capacity is evened out to an average value of $14.8 \mathrm{mM} / \mathrm{pH}$ in the $\mathrm{pH}$ interval from 3 to 11 , which is a considerable improvement over the simple phosphate buffer (Figure A1b). When mass spectrometry is used to measure concentrations, the present of phosphate is problematic. Also, if the sample contains 1,2-diol groups, the boric acid may form covalent bonds with them. It can be noted that the ionic strength increases ten-fold uniformly as the $\mathrm{pH}$ increase from 2 to 11 (0.02 to $0.2 \mathrm{M})$, which should not be a problem in data analysis [22]. Generally, the Britton-Robinson buffer can be highly recommended, provided it is compatible with the detection method and the chemistry of the sample.

\section{Minimalist Universal Buffer (MUB)}

Finding ways to avoid the chloride may be a good feature when studying weaker salt formers of drugs. In cases where chloride, borate, and phosphate need to be avoided, the "AEM-10.10.30" (devised here) may be a 
suitable universal buffer (Figure A1e). The AEM buffer consists of $10 \mathrm{mM}$ acetic acid, $10 \mathrm{mM}$ ethylenediamine, and $30 \mathrm{mM}$ mesylic acid, which is used to adjust the starting $\mathrm{pH}$ to about 1.7. The buffer capacity is nearly as uniformly distributed as in the case of the Britton-Robinson buffer, but a lower level is chosen (average of 4.4 $\mathrm{mM} / \mathrm{pH}$ over $\mathrm{pH} 3-11)$. This buffer capacity would allow the commercial $\mathrm{p} K_{\mathrm{a}}$ analyzers to easily set $\mathrm{pH}$ in increments of 0.2 across the $\mathrm{pH}$ range, using $0.5 \mathrm{M}$ titrants. In that sense, the concentrations of buffer components are "minimalist" - being just right for the titration equipment used. Because the universal buffer is a combination of an acid and a base, the ionic strength remains nearly constant across the working $\mathrm{pH}$ range, which is unique among the common universal buffers, and is particularly well suited for solubility applications. A buffer similar to the AEM, consisting of lactic acid and ethylenediamine, has been successfully tested [114].

As oil, ethylenediamine may not be convenient to work with. To remain chloride-free, alternatively, it may be useful to form a dimesylate or diacetate salt from ethylenediamine free base and either mesylic or trifluroacetic acid.

\section{Mass Spectrometry-Friendly Minimalist Universal Buffer (MS-MUB)}

In the AEM-10.10.30 buffer design, mesylic acid is proposed to set the initial pH to 1.7. However, mesylic acid is not compatible with mass spectrometer use. AET-25.25.75 (25 mM acetic acid, $25 \mathrm{mM}$ ethylenediamine, $75 \mathrm{mM}$ trifluroacetic acid) was formulated to be MS-friendly, higher-capacity version of the AEM buffer. Figure A1f shows the buffer capacity and ionic strength as a function of pH capacity, with average values of $8.3 \mathrm{mM} / \mathrm{pH}$ and $0.096 \mathrm{M}$, respectively. Excessive dilution effects due to titrant addition would not favor higher buffer capacity versions.

\section{Titration Curves and the Incrementing of $\mathrm{pH}$ by Titrant Additions}

Figures $\mathbf{A} 2$ show the titration curves for four of the above cases. Figure $\mathbf{A 2 a}$ shows that the $\mathrm{pH}$ adjustment across the steep pH 3-11 region in the "blank" titration would be difficult to achieve with normal titration equipment and titrant concentrations, if $0.2 \mathrm{pH}$ increments were desired. By selecting the $50 \mathrm{mM}$ phosphate buffer, the steepness in the working $\mathrm{pH}$ range is lessened, and it should be possible to control $\mathrm{pH}$ adjustment with the usual equipment. It gets much easier with the virtually linear titration curve produced with the Britton-Robinson universal buffer (Figure A2c). The linearized titration curve in Figure A2d for the minimalist universal buffer, AEM-10.10.30, is nearly as attractive as that of the Britton-Robinson buffer. AET-25.25.75 looks similar to the AEM buffer.

\section{Possible Uses of the Minimalist Universal Buffer}

Figure A3 shows the expected titration curve for haloperidol (free base) in AEM-10.10.30 buffer, titrated with $0.5 \mathrm{M}$ mesylic acid (cf., Figure 3a). Haloperidol itself provides good buffering below $\mathrm{pH} 5$. Above $\mathrm{pH}$, the titration curve would have been too steep, were it not for the buffering action of the AEM buffer. The region from $\mathrm{pH} 5$ to 12 is sufficiently buffered, so that $\mathrm{pH}$ increments of 0.2 could be achieved by $0.5 \mathrm{M}$ titrant minimum volume additions. This is an example of a "minimalist" buffer, which provides a boost where needed, but otherwise stays minimally intrusive. 
(a) Distilled Water

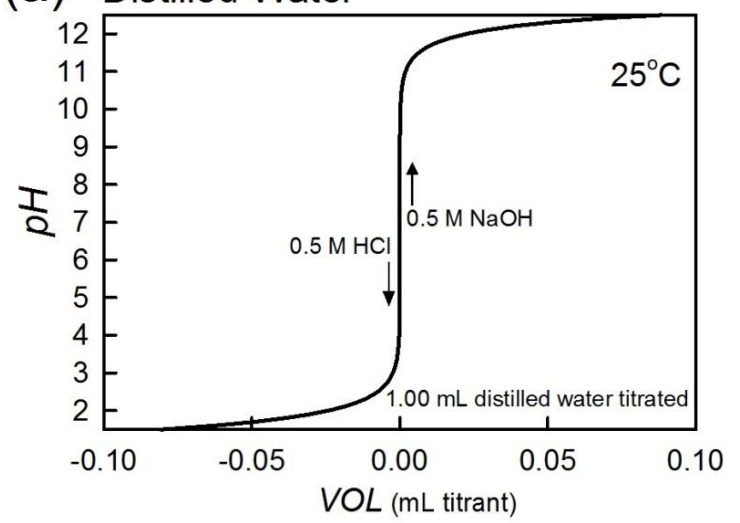

(C) Britton-Robinson Buffer APB-40.40.40

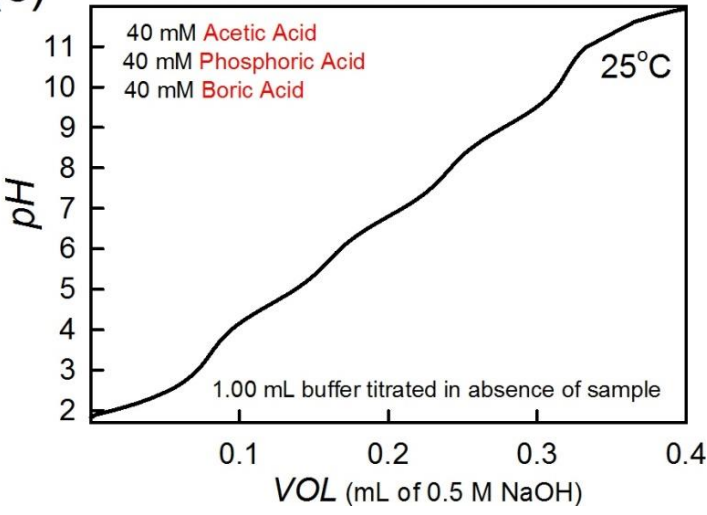

(b) Phosphate Buffer USP-25NF Phosphate

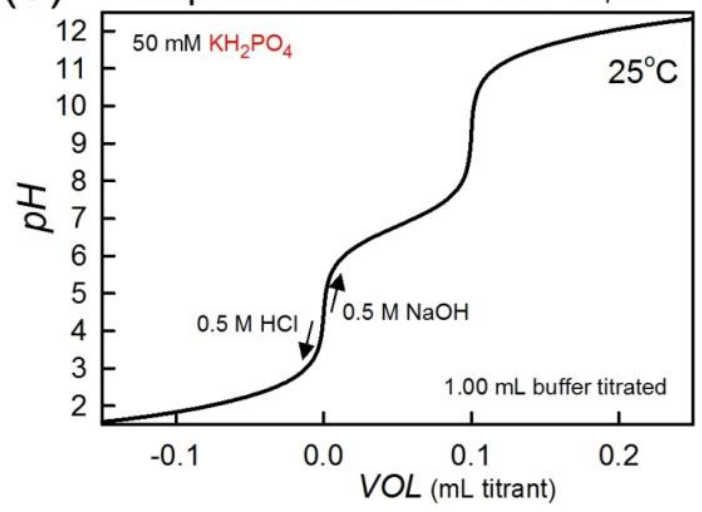

(d) Minimalist Solubility Buffer AEM-10.10.30

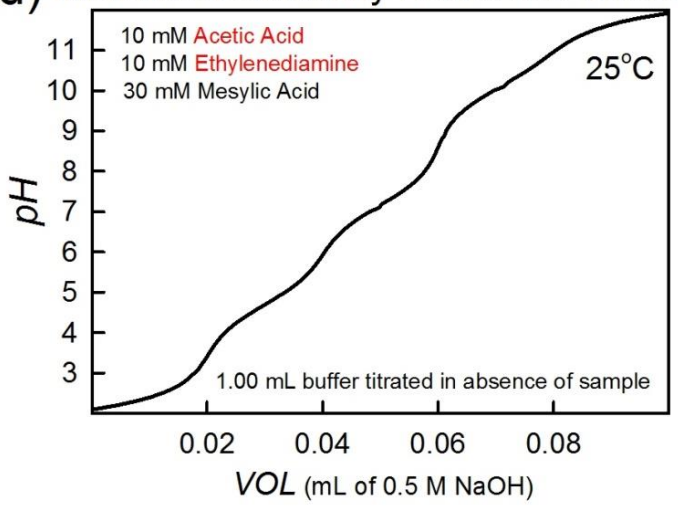

Figure A2. Titration curves for four of the examples in Figure A1. See text.
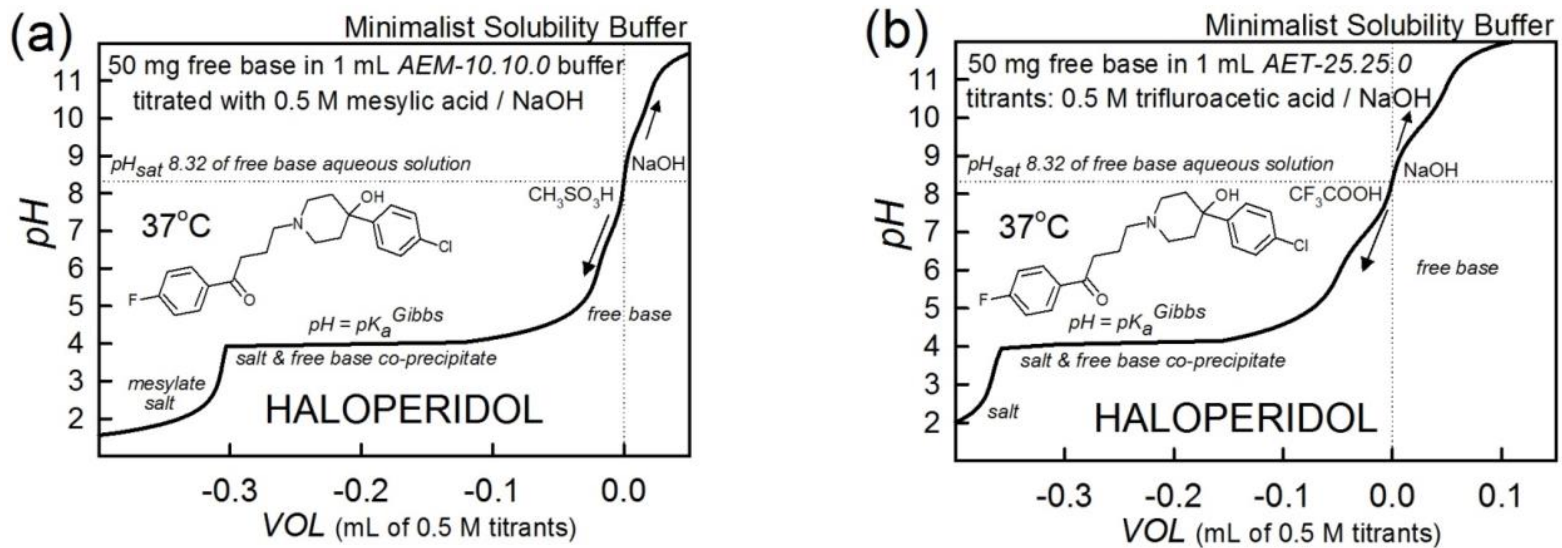

Figure A3. Examples of titrations of haloperidol in the AEM minimalist buffer at two different concentrations of buffer components. 


\section{Appendix B - Simple Henderson-Hasselbalch Equations}

The relationship between solubility and $\mathrm{pH}$ can be easily derived for a given equilibrium model. For example, in the case of a simple monoprotic base, a saturated solution can be defined by two equations and the corresponding constants:

$$
\begin{array}{ll}
\mathrm{BH}^{+} \leftrightarrows \mathrm{H}^{+}+\mathrm{B} & K_{\mathrm{a}}=\left[\mathrm{H}^{+}\right][\mathrm{B}] /\left[\mathrm{BH}^{+}\right] \\
\mathrm{B}(\mathrm{s}) \leftrightarrows \mathrm{B} & S_{0}=[\mathrm{B}]
\end{array}
$$

$S_{0}$ is the intrinsic solubility (of the free base at $\mathrm{pH} \gg \mathrm{p} K_{a}$ ). At a particular $\mathrm{pH}$, solubility is defined as the mass balance sum of the concentrations of all of the species dissolved in the aqueous phase:

$$
S=\left[\mathrm{BH}^{+}\right]+[\mathrm{B}]
$$

The square brackets denote molar concentration of species (at the constant ionic medium reference state [27]). The above equation is usually converted into an expression containing only constants and $\left[\mathrm{H}^{+}\right]$(as the sole variable), by substituting the ionization and solubility Eqs. B.1 into Eq. B.2.

$$
\begin{aligned}
S & =\left[\mathrm{H}^{+}\right][\mathrm{B}] / K_{\mathrm{a}}+[\mathrm{B}] \\
& =S_{0}\left(\left[\mathrm{H}^{+}\right] / K_{\mathrm{a}}+1\right) \\
& =S_{0}\left(10^{\mathrm{pKa}-\mathrm{pH}}+1\right) \\
\log S & =\log S_{0}+\log \left(10^{+\mathrm{p} K \mathrm{a}-\mathrm{pH}}+1\right)
\end{aligned}
$$

The dashed curve in Figure 1a is a plot of Eq. B.4 for atenolol ( $K_{\mathrm{a}}$ 9.54). At the bend in the curve, the pH equals the $\mathrm{p} K_{\mathrm{a}}$. For $\mathrm{pH} \gg \mathrm{p} K_{\mathrm{a}}$, the equation represents a horizontal line: $\log S \approx \log S_{0} ;$ for $\mathrm{pH}<<\mathrm{p} K_{\mathrm{a}}$, the equation is a diagonal line with slope -1 .

Other cases may be similarly derived. Table B.1 is a collection of solubility equations for such simple cases, with up to two $\mathrm{p} K_{\mathrm{a}}$ values.

Table B.1. Solubility-pH Equations for Mono- and Diprotic Molecules

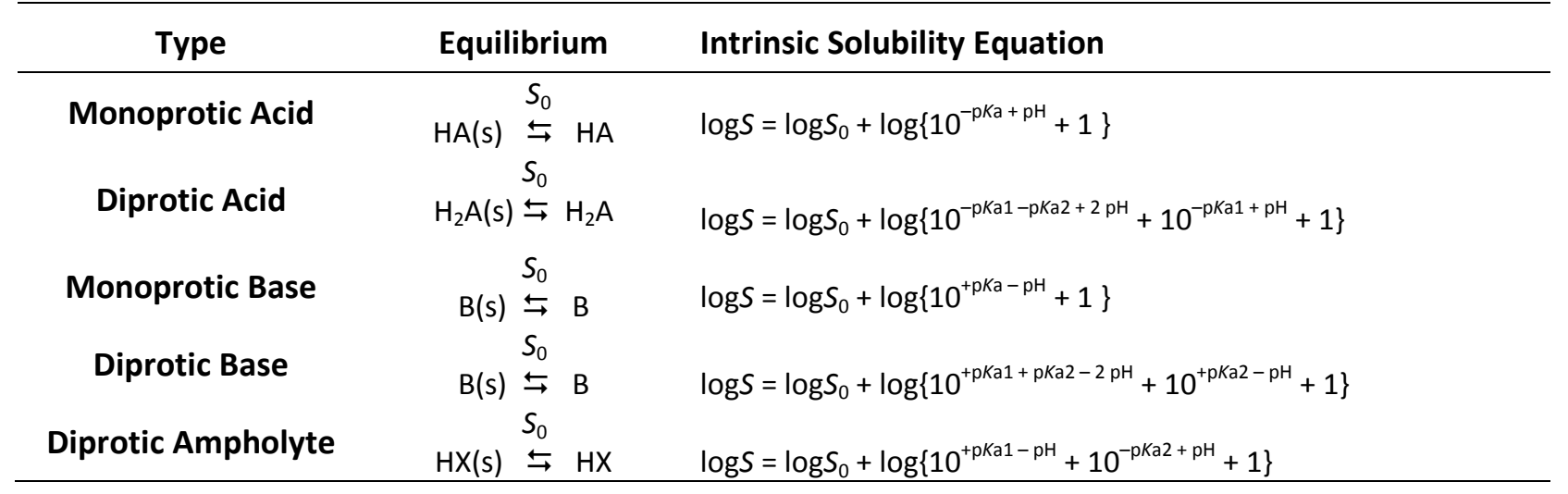




\section{Appendix C - The Four-Parameter Electrode Calibration Procedure}

In highly-developed potentiometric methods for $\mathrm{p} K_{\mathrm{a}}$ determination, research-grade combination $\mathrm{pH}$ electrodes are calibrated to cope with large swings in ionic strength, over a wide range of pH 0.5-13.5 [27]. Some of the calibration procedures can be translated to $\mathrm{pH}$ reading in solubility-pH experiments. The voltage read by a $\mathrm{pH}$ meter needs to be converted to ' $\mathrm{pH}^{\prime}$ on what is called the operational scale. In the two-part procedures of the Pion Inc and Sirius Analytical Ltd $\mathrm{p} K_{\mathrm{a}}$ analyzers, the $\mathrm{pH}$ electrode is first 'calibrated' before each assay with a single aqueous $\mathrm{pH} 7.00$ buffer(traceable to the NIST phosphate buffer at $25{ }^{\circ} \mathrm{C}, \mathrm{pH} 6.865$ [155]), with the ideal Nernst slope assumed to be $59.16 \mathrm{mV} / \mathrm{pH}\left(25^{\circ} \mathrm{C}\right)$. Then this operational $\mathrm{pH}$ is 'standardized' to a concentration-based $\mathrm{p}_{\mathrm{c}} \mathrm{H}$ scale (i.e., $-\log \left[\mathrm{H}^{+}\right]$), done on a weekly basis. Both steps are carried out under thermostated conditions (most commonly at $25 \pm 0.1^{\circ} \mathrm{C}$ ).

Equilibrium quotients in currently practiced $\mathrm{p} K_{\mathrm{a}}$ and solubility procedures employ the constant ionic medium activity scale where $\mathrm{p}_{\mathrm{c}} \mathrm{H}$ rather than $\mathrm{pH}$ is applied. This is a valid thermodynamic activity scale, where the limiting state is the ionic strength-adjusted solution (e.g., $0.15 \mathrm{M} \mathrm{KCl}$ ), rather than pure water. For many years, the $\mathrm{pH}-$ to $-\mathrm{p}_{\mathrm{c}} \mathrm{H}$ standardization has been based on the equation below $[27,45,111-113]$,

$$
\mathrm{pH}=\alpha+k_{\mathrm{s}} \cdot \mathrm{p}_{\mathrm{c}} \mathrm{H}+j_{\mathrm{H}} \cdot\left[\mathrm{H}^{+}\right]+j_{\mathrm{OH}} \cdot K_{\mathrm{w}} /\left[\mathrm{H}^{+}\right]
$$

where $K_{\mathrm{w}}$ is the ionization constant of water, $K_{\mathrm{w}}=\left[\mathrm{H}^{+}\right]\left[\mathrm{OH}^{-}\right]$(concentration scale, molarity). At $25^{\circ} \mathrm{C}$ and 0.15 $\mathrm{M}$ ionic strength, $\mathrm{p} K_{\mathrm{w}}=13.764$. The four parameters $\left(\alpha, k_{\mathrm{s}}, j_{\mathrm{H}}, j_{\mathrm{OH}}\right)$ are determined by a weighted least-squares procedure using data from alkalimetric "blank" titrations of known concentrations of $\mathrm{HCl}$ [45]. Typical aqueous values of the adjustable parameters are shown in Table C.1. Such a standardization scheme extends the range of accurate $\mathrm{pH}$ measurements and allows $\mathrm{p}_{\mathrm{a}}$ values to be assessed as low as 0.6 (caffeine) and as high as 13.3 (salicylic acid) [27].

Table C.1 Typical four parameter electrode standardization parameters ${ }^{\mathrm{a}}$

\begin{tabular}{cccccc}
\hline $\begin{array}{c}\text { Temperature } \\
\left({ }^{\circ} \mathbf{C}\right)\end{array}$ & $\begin{array}{c}\text { Ionic Strength } \\
(\mathbf{M})\end{array}$ & $\boldsymbol{\alpha}$ & $\boldsymbol{k}_{\mathrm{S}}$ & $\boldsymbol{j}_{\mathrm{H}}$ & $\boldsymbol{j}_{\mathrm{OH}}$ \\
\hline 25 & 0.00 & 0.070 & 0.9971 & 0.8 & -0.6 \\
$\mathbf{2 5}$ & $\mathbf{0 . 1 5}$ & $\mathbf{0 . 0 9 0}$ & $\mathbf{1 . 0 0 1 9}$ & $\mathbf{0 . 5}$ & $-\mathbf{0 . 5}$ \\
25 & 1.00 & 0.109 & 0.9935 & -1.2 & 0.0 \\
\hline 37 & 0.00 & 0.070 & 0.9972 & 0.3 & -0.8 \\
37 & 0.15 & 0.091 & 1.0017 & 0.0 & -0.7 \\
37 & 1.00 & 0.109 & 0.9933 & -2.7 & 0.2 \\
\hline
\end{tabular}

$\bar{a}$ Each pH electrode may have its own distinct parameters, which need to be checked on a weekly basis during periods of active use [27].

The intercept constant, $\alpha$, is ideally defined by $-\log f_{\mathrm{H}}$, where $f_{\mathrm{H}}$ is the activity coefficient of the $\mathrm{H}^{+}$(i.e., activity of $\left.\mathrm{H}^{+},\left\{\mathrm{H}^{+}\right\}=0.81\left[\mathrm{H}^{+}\right]\right)$. In practice, a may also serve to mop up other effects not dependent on $\mathrm{pH}$. The slope factor, $k_{S}$, is used to take into account that a particular $\mathrm{pH}$ electrode may not have the ideal $100 \%$ Nernstian slope. Liquid junction potentials that form in very low ionic strength solutions can be slightly $\mathrm{pH}$-dependent, an effect that propagates into the $k_{\mathrm{S}}$ empirical slope factor parameter [27]. The $j_{H}$ term corrects $\mathrm{pH}$ readings for the nonlinear $\mathrm{pH}$ response due to the liquid junction and asymmetry potentials in acidic 
solutions $(\mathrm{pH}<2.5)$. The $j_{\mathrm{OH}}$ term corrects for alkaline $(\mathrm{pH}>11.5)$ nonlinear effects, due to liquid junction potentials. The latter parameter may also compensate for 'sodium error' when $\mathrm{NaCl}$ is used to adjust the ionic strength and/or when $\mathrm{NaOH}$ is the titrant.

In a typical "blank" titration procedure to determine the four parameters, about $0.9 \mathrm{~mL}$ of $0.5 \mathrm{M} \mathrm{HCl}$ is added to $20 \mathrm{~mL}$ of $0.15 \mathrm{M} \mathrm{NaCl}$ (or $\mathrm{KCl}$ ), to lower the $\mathrm{pH}$ to 1.8 . The solution is then carefully titrated with standardized $0.5 \mathrm{M} \mathrm{NaOH}$ (or $\mathrm{KOH}$ ) up to about $\mathrm{pH} 12.2$ (which consumes about $2.0 \mathrm{~mL}$ of the titrant). Figure A2a shows such a plot. Since the ionization constant of water is known over a wide range of conditions [158] and since the concentrations of $\mathrm{NaOH}$ and $\mathrm{HCl}$ in the titrated solution are also precisely known at each point, then the hydrogen ion concentration, $\left[\mathrm{H}^{+}\right]$, can be calculated, so that at each point in the titration, both the operational $\mathrm{pH}$ and the concentration $\mathrm{p}_{\mathrm{c}} \mathrm{H}$ are known. The parametric relationship between the two scales can be deduced by least-squares fitting of the calculated $\mathrm{p}_{\mathrm{c}} \mathrm{H}$ titration curve to the observed $\mathrm{pH}$ titration data points. The typical values shown in Table C.1 (e.g., for $25^{\circ} \mathrm{C}, I=0.15 \mathrm{M}$ ) can vary substantially from electrode to electrode: $\alpha=0.08-0.15, k_{\mathrm{S}}=0.995-1.005, j_{\mathrm{H}}=0.5-1.0$ or higher, and $j_{\mathrm{OH}}=-0.5$ or lower. For a given electrode the precision of the four parameters are typically $\pm 0.02, \pm 0.002, \pm 0.2$, and \pm 0.4 , respectively.

When the differences $\mathrm{pH}-\mathrm{p}_{\mathrm{c}} \mathrm{H}$ in blank titrations are examined as a function of $\mathrm{pH}$, the variance in the interval pH 4-9 can be very large, as is expected of the unbuffered neutral pH region. Regression determination of the four parameters to such data requires an appropriate weighting scheme [159].

Besides the determination of the four parameters, the blank titration quality controls two other experimental variables. (a) The volume of standardized $\mathrm{NaOH}$ needed to neutralize the added $\mathrm{HCl}$ determines the $\mathrm{HCl}$ concentration very precisely. (b) $\mathrm{CO}_{2}$ concentration in the $0.15 \mathrm{M} \mathrm{KCl}$ and in the $\mathrm{NaOH}$ titrant can be precisely determined, since the neutral $\mathrm{pH}$ region clearly reveals the $\mathrm{p} K_{\mathrm{a}}$ profile of carbonic acid. 


\section{Appendix D - Questionnaire Summary}

Ten Participants from:

Doing solubility meausurements:

Attended panel discussion:
6 industry $\_$_ academia $\_$regulatory agency _ 0 _other

8 regularly _ 1 occasionally 1 n never

9 yes 1 n no

In various topic areas (italics below), statements were posed (underlined below), and the participants were asked if they agreed (YES), disagreed (NO), or were unsure (?) regarding the statement. The counts of responses are indicated in brackets at the beginning of the statement. Additional comments are in quotation marks and highlighted in yellow.

Solubility Units, Tabulation of Results and the Use of Logarithmic Plots

[9YES, $1 \mathrm{NO}$ ] Solubility needs to be tabulated both in molarity and in practical $(\mathrm{mg} / \mathrm{mL}$ or $\mu \mathrm{g} / \mathrm{mL})$ units. Logarithm should be expressed in molar concentrations.

"Molarity scale is more appropriate for me as a chemist. MW should be indicated together with molar solubility."

"I would not recommend to use $S$ in $\mu \mathrm{g} / \mathrm{mL}$ and logS in molar unit. As for me I would always use the molar concentration for the solubility measures. It would force people to characterize the solid form (salt? Not salt, partial) a bit more rigorously. In the worst case scenario we should express solubility in both units!"

[7YES, 3NO] Standard deviations in measured solubility (based on averaging 3 or more values) should be presented.

[9YES, 1NO] Additionally, a graphical display of log $S$ vs. $\mathrm{pH}$ (but not $S$ vs. $\mathrm{pH}$ - unless tabulated numbers are also available) would be visually helpful.

Solubility Methodology and the Benefits of Knowing the Accurate $p K_{a}$

[10YES] The "gold standard" multiple-pH buffer shake-flask measurement is recommended for challenging ionizable molecules.

"[I would agree] as long as "shake-flask" is broadly understood - in situ UV concentration monitoring, for example, is a "shake-flask" measurement of sort."

Which other methods may also be satisfactory (with the Henderson-Hasselbalch relationship independently validated):

[3 CheqSol; 2 potentiometry; 1 direct microscopic obs. of drop; 1 PXRD; 1 dissolution calorimetry]

[10YES] Miniaturization $(0.2-2 \mathrm{~mL})$ can be recommended, as long as the protocols are rigorous and well validated.

[10YES] For ionizable molecules, the measurement of $S_{w}$ without measuring the equilibrium $\mathrm{pH}$ is not 
recommended.

"Water solubility may be of practical interest, but it must be complemented, not substitute the procedure described above."

[10YES] It is far better to measure $S_{\mathrm{pH}}$ values in well-qualified buffers, at 3 or more equilibrated $\mathrm{pH}$ values, symmetrically bracketing the $\mathrm{p} K_{\underline{a}}$.

[8YES, 2NO] The $\log S-\mathrm{pH}$ data should be evaluated to estimate the value of the intrinsic solubility, $\underline{S}_{0}$.

[10YES] Solubility is a function of temperature, so the actual assay temperature needs to be reported.

"Measurements at multiple temperatures are highly desirable, since they may indicate also at important changes in the dissolution mechanism."

"Graphs vs. T are also helpful."

[6YES, 4?] If solubility is measured at several different temperatures, it is normal to report solubility in either mole fraction ( $\mathrm{x}_{2}$ ) or molality (mol/kg solvent) units. It would be very helpful also to include the solubility in molarity units (mol/L solution).

"We think it is better to provide Molar solubility always, indicating the temperature of each measurement."

[10YES] Measured solubility can be affected by ionic strength (particularly when salt solubility is measured), so the ionic strength usually needs to be reported. It is easy to calculate the ionic strength coming from the buffers and the adjustors, but it is not evident how to contemplate the contribution of the own sample.

\section{Equilibration Time and Stirring Protocol}

[9YES, 1?] Ordinarily, the shake-flask method determines the equilibrium solubility of the most stable solid state form of the compound (which may be different from the original solid form added to the suspension). "I am not sure about 'the most stable form,' but the two forms may immensely differ."

"It is recommended to prove this by alternative method, DSC, or PXRD."

"It would be beneficial to confirm the solid state after solubility measurements especially if the measurement relies on precipitation."

[6YES, 3NO, 1?] Probably $24 \mathrm{~h}$ is a good average equilibration time for the uncharged drug form equilibration, but when measuring the equilibrium solubility of practically-insoluble drugs it is a good idea to test longer times (e.g., 48-72 h). In regions of $\mathrm{pH}$ where the sample molecule is predominantly ionized (e.g., salt solubility applications), equilibration times as short as 1-6 $\mathrm{h}$ may be adequate.

"pH control is a good tool to see if equilibrium has been reached."

"It is helpful to have measurement during the incubation time."

[5YES, 3NO, 2?] The $25 \%$ stir $+75 \%$ sedimentation timing protocol (Baka et al. J. Pharm. Biomed. Anal. 2008, $46,335-341)$ is recommended. Stopping the stirring and allowing the solid to sediment contributes to formation of better quality crystals, consistent with a truly equilibrated solution. 
"It could be a different ratio."

[5YES, 3?, 2NO] In regions of $\mathrm{pH}$ near the $\mathrm{p} K_{\mathrm{a}}^{\text {Gibbs }}$ (sometimes called ' $\mathrm{pH}$ max'), where the salt form plus the uncharged form of the drug co-precipitate (e.g., Serajuddin and Rosoff 1984; Serajuddin and Jarowski 1984, 1985, 1993), longer equilibration times (e.g., 24 ]-48 h) may be necessary due to the tendency of the solutions becoming supersaturated.

[3YES, 4?, 3NO] For some kinds of drug (e.g, phenothiazines), at temperatures above the Krafft point, the solid may dissolve directly into a micellar or a highly supersaturated form, which may, in turn, transition into an oil (depending on $\mathrm{pH}$ ), longer equilibration times (e.g., 48-72 h) may be necessary if equilibrium solubility is to be measured.

"These kinds of compounds may go through liquid-liquid phase separation and additional equilibration time will not lead to solid form precipitation."

[3YES, 3?, 4NO] Equilibration times as long as many days have been used. Ordinarily, long times are not recommended, unless one is interested to determine the solubility enhancement of a biorelevant medium or human intestinal fluid.

"Long times can be helpful to follow re-crystallization of alternative solid forms."

"Equil. time should be as long as necessary to provide precipitation of the most stable polymorph."

\section{Shortening the Equilibration Time}

[2YES, 3?, 5NO] If the initial solid form of the drug is unknown, dissolve the crystals in the assay vial with a drop or two of methanol (or another volatile organic solvent in which the crystals dissolve). Swirl the vial to let the methanol evaporate before adding the solution medium. Usually the freshly-formed crystals wet better and dissolve quickly. This procedure is often used in the DTT method.

"The choice of solvent depends on the substance."

"Suitable for selection study of only candidate."

[4YES, 3?, 3NO] If the initial solid form of the drug is unknown, add the crystals to the buffer solution. For a weak acid/base drug, add a known amount of standardized base/acid titrant to dissolve the solid, but avoid extremes of $\mathrm{pH}$. Quickly add a known amount of standardized counter titrant to the desired starting $\mathrm{pH}$. A procedure like this is used in the CheqSol method, with repeated cycles of dissolution and re-precipitation by pH adjustment.

"Method suitable for fast solubility determination."

[2YES,5?,3NO] The Loftsson et al. (Int. J. Pharm. 2005, 302, 18-28) temperature spiking cycles and seed crystal may be used to shorten the equilibration time.

"Careful analysis of the solid phase and microscopy observations are needed for correct interpretation of results."

[1YES, 6?, 3NO] The Facilitated Dissolution Method of Higuchi (cf., Avdeef [4]), consisting of adding a drop of water-immiscible oil to the solution medium, may be used to speed up the rate of equilibration. The method is 
fundamentally rigorous and deserved much more attention than it has received, including validation studies. "This is an interesting approach that have not been much appreciated. We'd love to try it out."

[1YES, 8?, 1NO] Most of the above methods look promising.

\section{Solution and Solid State Composition}

[9YES, 1NO] It is a good idea to keep the assay solutions simple and to define all components precisely. Report the state of the solid, if known (e.g., polymorphic, hydrate, solvate, amorphous, stereoisomer).

"It is a must to characterize the solid samples as detailed as possible (structure, meso-structure, particle size, shape, surface area, etc.), also in the process of dissolution recrystallization."

"Time and resource consuming. Not be used for selection."

[10YES] When possible, it is a good idea to do solid state characterization of the precipitate at the end of equilibration, to determine if a solid-state transformation had taken place, or to confirm the crystalline nature of the solid, or to ensure that a mixture of solid forms is not present.

"At the beginning, at the end, at the intermediate stages; "unusual" temperature dependence of solubility also suggests a change in the solid form."

[10YES] When studying salt solubility, it is particularly helpful (and sometimes necessary) to specify the actual weight of sample in each vial. (It can be unhelpful to see statements such as "excess solid was added.")

"Actual weight should always be specified."

[9YES, 1NO] It is not enough to state that "water was used" as the solvent. Was there any added ionic strength adjustor (e.g., $0.15 \mathrm{M} \mathrm{NaCl}$, etc.)? Was carbon dioxide purged out? Low soluble bases such as terfenadine can indicate solubility over several magnitudes due to the effect of ambient $\mathrm{CO}_{2}$ in unbuffered solutions.

"All details of the method, procedure, have to be described."

pH Measurement Using Glass Electrodes

[10YES] It is highly recommended that the final $\mathrm{pH}$ of the saturated solution be carefully measured, using a properly standardized electrodes. (It is a risky practice to assume that the assay buffer $\mathrm{pH}$ is not altered by the addition of sample.)

"Besides final $\mathrm{pH}$, value of the beginning and, for example, after stirring, should be indicated."

[10YES] Particular attention should be given to the $\mathrm{pH}$ electrode calibration, especially when extreme $\mathrm{pH}$ is measured $(\mathrm{pH}<1$ or $\mathrm{pH}>12)$ or when the ionic strength reaches high values, as in the case of salt solubility measurement.

[7YES,3?] State whether the reported $\mathrm{pH}$ is on the "operational" or the "concentration" scale. The "mixed" scale is not recommended. 
"The definition of operational $\mathrm{pH}$ and mixed $\mathrm{pH}$ scales should be provided. If mixed scale refers to the measurement of $\mathrm{H}^{+}$ in activities and the rest of species in concentrations, we don't see why the mixed scale is not recommended if everything is well specified."

"Operational [is preferred]."

[6YES, 4?] A strong case can be made that general solubility equilibrium quotients are best formulated in the concentration scale (rather than activity), with $\mathrm{pH}$ electrodes standardized in a constant ionic medium, e.g., $0.15 \mathrm{M} \mathrm{NaCl}$. This may be especially important when salt solubility measurements are performed.

"We have never done that, but can be a good idea."

[8YES, 1?, 1NO] State how pH was adjusted (e.g., $0.1 \mathrm{M} \mathrm{HCl}, 1 \mathrm{M} \mathrm{NaOH}$, etc.). Statements like: "concentrated $\underline{\mathrm{H}}_{3} \underline{\mathrm{PO}}_{4}$ was added drop-wise to adjust the $\mathrm{pH}^{\prime \prime}$ are not clear enough. It is far better to state the precise concentration and volume of the titrant used to adjust the $\mathrm{pH}$ to the desired starting point.

"We agree with the comment."

Buffers

[10YES] It is inadequate to state that "buffers were used". State precisely which buffer compounds were used. Problems with specific buffers can occur so it is important to specify the nature.

[10YES] State which salt forms of buffers were used (e.g., $\mathrm{KH}_{2} \underline{P O}_{4}{ }_{2} \mathrm{Na}_{2} \underline{H P O}_{4} \cdot \underline{2} \underline{H}_{2} \mathrm{O}$ ).

[9YES, 1NO] State the concentrations of the buffers, since this will be needed to calculate the total ionic strength. Ordinarily, relatively low buffer concentrations (e.g., 5-10 mM) can be recommended.

"10 $\mathrm{mM}$ is not enough to buffer solutions of compounds with relatively high solubility"

[6YES, 3?, 1NO] Some buffers have inappropriately high UV absorptivity, if concentration is determined by UV spectroscopy. List some examples of buffers to avoid.

"We normally use HPLC for compound quantification."

"HEPES, phosphate buffer(s) [are to be avoided] if [sample] substance absorbs below 240-250 nm."

Separating Solid from Saturated Solution

[7YES, 2?, 1NO] Filtration is recommended, using hydrophilic filters, typically 0.2-0.45 $\mu \mathrm{m}$ pore sizes. "As Krisztina said, it depends on the $p K_{a}$ of the compound and also its $\log P . "$

"Type of filters may influence the So value; maybe several types of filters should be used (depending on the $p K_{a}$ value and pH of solution)."

"Filtration is very time consuming and for industry it is preferred to do centrifugation. We have performed centrifugation for a 5 or 6 years and no problem was encountered yet. Perhaps some micellar problems were encountered but the detection of this is not always clear. A better way to find those problems could be advisable."

[8YES, 2?] It is useful to discard the first $10-25 \%$ filtered solution, to allow filters and vial surfaces to be saturated with adsorbed compound. The filtration step should not be rushed. 
"One must take care that no change in the solid form occurs during filtering (possible for highly polymorphic [prone] solids)."

[6YES, 3?, 1NO] If colloidal dispersions or "promiscuous inhibitors" (McGovern et al. J. Med. Chem. 2002, 45, 1712-1722) are suspected, then the filtered solutions may be further subjected to ultracentrifugation.

[7YES, 2?, 1NO] It is highly inadvisable to centrifuge first and then filter.

\section{Additional Comments/Suggestions}

"A mass spectrometer can be very helpful in cases of low sensitivity of other techniques or to corroborate the presence of aggregates, etc."

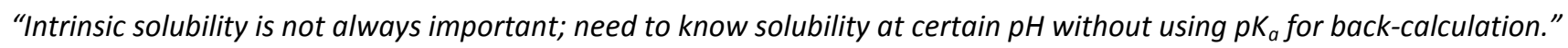

"Generally: my early-research group analyzes 15,000 cpds/annum in a high throughput type assay (initial dissolution in DMSO followed by addition to aqueous buffer, $24 \mathrm{~h}$ shaking, filtration, HPLC) and approximately $1000 \mathrm{cpds} /$ annum in a shake flask assay (solid compound + buffer or formulary solvents/various pHs), 24 h stirring, centrifugation, HPLC). Many of the suggestions given in the questionnaire are very interesting but definitely not feasible in an industry lab which has to provide the researcher with data as fast as possible and as accurately as necessary. Early solubility data very often serve as estimation for the pharmacologist and as comparison of different clusters within HT Lab. They don't need exact numbers but rather a hint if the compound could have an solubility issue during further activities."

"I am responsible for the PhysChem Lab in the Medicinal Chemistry - Global Drug Discovery. We have to measure the solubility of about 12,000 new compounds every year to support our colleagues in the different projects. They don't need absolutely exact solubility results at this time of research; they need the results very fast for a ranking between the several compounds. Thus some of the ideas and questions below are very good from the scientific point of view but not feasible for our high throughput measurements. In such cases I put a star $\left({ }^{*}\right)$ in the space following the statement."

"It would be very helpful to have two kind of operational suggestions: (1) a quick ones for a more routine work; (2) a more complete suggestions to be applied when compound is already in candidate profiling. Quick results must be obtained at the physchem departments in the lead optimization phase while in the candidate profiling more time and compound amount can be devoted to study the solubility of the compound."

"Although we are measuring HT solubility in a pragmatic way (DMSO 5\% precipitative solubility), [such measurements] are still useful in drug discovery. We can select the most soluble series, we could even build some sort of in silico guide for chemists using half a million compounds' solubility data ( $\mathrm{pH} 7.4,1 \mathrm{~h}$ equilibration $0.1 \mathrm{M}$ phosphate buffer) expressed in molar term using the matched molecular pairs approach. So we have a long lists of substituents with the average contribution to solubility (enhance or reduce) and its standard deviations. The pharmaceutical industry is interested in measuring solubility in various bio-relevant media such as Simulated Lung Fluid, Simulated Intestinal fluid, etc. I think even for those measurements the $\mathrm{pH}$, solid form characterization, temperature etc are important details to provide. Overall, it was an exciting session and I was amazed how many people were there until nearly midnight!"

"Solubility measurement methodology depends on sample quantity availability, precision of measurement and characteristics of the drug molecule (purity, solid state properties)."

"[Reporting] the amount of solid in excess is important, especially when the solid is a salt, as the counterion may affect pH of the solution significantly."

"Shaking intensity may be more important than time of incubation." 
"I do not like people saying "24 $h$ " [incubation time]. We have reached equilibrium at $4 h$ in some cases.

"I was really interested in learning yesterday on the importance of sedimentation on solubility measurements. If you have a relevant paper PLEASE could you send it to me?"

"Buffer species identity: We have actually observed effects (Vertzoni et al. JPP, 2004) when the API (if ionized) interacts with the oppositely charged (?) species of the buffer system."

Extra Topic Questions - Describe How You Handle The Following Potential Challenges

poor wettability

"High stirring power during a long time."

目 adding a very small weight (e.g., $1-10 \mu \mathrm{g})$ of drug to a vial

[No comments.]

囯 formation of drug aggr./oligomers (dimers, trimers, ...), micelles, or drug-buffer complexes

"Shake-flask S-pH profiles"

专 formation of ion-pair between ionic str. adjustor (e.g., $\mathrm{NaCl}$, etc.) and charged form of drug

"Shake-flask S-pH profiles"

目 effect of impurities, especially those which are ionizable

"Not contemplated"

毗 compound decomposition at the extremes of $\mathrm{pH}$ or over long saturation times

"HPLC measurements provide an idea of decomposition, so we know that additional processes occur at that pH values."

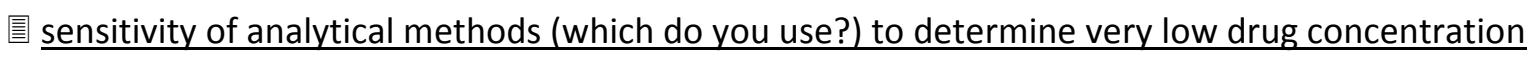

"HPLC"

\section{$Q \& A B \log$}

Q: Firstly, I remember that someone said at the conference that an ultrasonic bath can be used for stirring/mixing during the solubility assay. Is the ultrasonic bath a good option for dissolving compounds over $6 \mathrm{~h}$, or is it better to do some stirring in the assays? An ultrasonic bath could be an aggressive way to dissolve compounds, to shorten subsequent stirring time. No problems with wetting should exist. Is it true?

A1: My guess is that some would think that sonic stirring might create a system that is supersaturated (if the starting material is a salt of the drug). The sedimentation period should help the suspension to go in the direction of equilibrium. Wetting could make the sonic agitation problematic. (Not sure.)

A2: I do not recommend it. If the compound is poorly wettable, the sonication will drive the solid out to the surface of the flask.

Q: One question is about basics of solubility assay. I have planned that I will add certain amount of compound in vial and then add buffer. After this when no precipitate exists, I will add more solid so long that some precipitate will appear. But if I use this protocol, then I'm not able to say how much compound I add to the solution. Is it better to perform some advance experiments before final experiment to get some information about solubility? Another possibility is to use DMSO stock solution of compound, but that creates another problem. If I need to add too much DMSO solution, then DMSO concentration in solution will go very high and then may influence solubility. So now I'm a little confused and I can't decide which is the better option. Which method do you recommend? 
A2: It is advisable to work out a general protocol at the start, which can be suitable for the majority of compounds (given non-problematic molecules). Someone new to solubility measurement needs to practice with easy compounds. A preliminary experiment is useful: try to dissolve $10 \mathrm{mg}$ solid in $1 \mathrm{~mL}$ solvent; if the solid is not fully dissolved, add an additional $9 \mathrm{~mL}$ of solvent $(1 \mathrm{mg} / \mathrm{mL})$. I would avoid adding $\mathrm{MeOH}$ or DMSO to the crystals. Rather I suggest to increase the stirring time and to change the speed from low to fast and back many times. After the preliminary work, the frequent difficulties in the case of poorly soluble compounds (problematic molecules) can be considered.

Procedures in the refined SSF protocol (Baka et al. JPBA, 2008) may be recommended:

- two-fold excess of solid

- control the $\mathrm{pH}$ after $1 \mathrm{~h}$ stirring, re-adjust if necessary

- incubation: $6 \mathrm{~h}$ stirring $+18 \mathrm{~h}$ sedimentation

- minimum of 3 replicates

A1: In principle, $p$ DISOL-X should be able to suggest how much solid to add, since solubility is first predicted in $p D I S O L-X$ and information about buffers exists. Most people will not use $p D I S O L-X$, so the guessing game will continue. My advice is to keep track of how much solid was added by weighting the vile before and after each addition of solid. This is more important in the salt region, since that is where supersaturation is most likely to occur, or where micelles and salt compete with one another for the compound. Please keep track of how much compound is added. I don't recommend using DMSO stock. (cf., Chen et al., Combi. Chem. HTS 2002, 5, 575-581). If solubility is a problem and if you need to prepare a stock solution, use methanol (or any other suitable volatile solvent). When the solution is added to the vial (before buffer is added), you can let the methanol completely evaporate, before adding buffer. But stay away from DMSO, if you are interested in measuring true aqueous solubility.

Q: Other question is about filtration. If I remember correctly, the best filter option is 0.2 or $0.45 \mu \mathrm{m}$ hydrophilic PVDF filter. Is it correct? Is it correct to use filter plate with vacuum manifold for the separation or better is use individual filters? I plan to perform experiments in $2 \mathrm{~mL}$ vials and then pipetting the solution to the filter plate. Is it good plan?

A1: See Kristina's poster about filters. My own preference is to use disposable filter tips that attach to syringes, $0.2 / 0.45$ $\mu \mathrm{m}$-pore hydrophilic PVDF. Draw in the suspension slowly, discard the first $25-50 \%$, and use the remainder for the assay. There is a lot more to be said about filtration.

A2: Yes, it is better to avoid filtration. If this is not possible, then select the right filter type and use 0.2 or $0.45 \mu \mathrm{m}$ pore. Discarding the first portion of filtrate reduces the error which can be caused by the adsorption to the filter. Never filter directly after agitation, keep the sedimentation/resting time allowing the system to reach the equilibrium. For very stable colloidal system ultracentrifugation can be a solution for phase separation.

Q: Next question is about buffer. Is it better to use universal buffer (same compounds as your slides: glycolic acid, acetic acid, MES, HEPES and taurine) or Britton-Robinson buffer for the solubility assay? Universal buffer should be better, but otherwise Britton-Robinson buffer [comprises fewer components]. Which buffer do you recommend?

A2: I think the use of the same buffer in wide $\mathrm{pH}$ range (like Britton-Robinson) is an advantage, since all the possible effects of the buffer components should be the same. This acetate-phosphate-borate buffer provides enough buffer capacity and no interactions have been experienced by us.

A1: My own inclination is not to use "off-the-shelf" buffer recipes. Select a buffer for the job. Keep the solution as simple as possible, and avoid unintended buffer-drug interactions. Also, keep the ionic strength controlled. Of course, that requires some experience. There is a lot more to be said about buffers.

Q: Last question is about ionic strength. Ionic strength of buffer should be $0.15 \mathrm{M}$. Is it better use for fixation $\mathrm{KCl}$ or $\mathrm{NaCl}$ ? Usually $\mathrm{NaCl}$ has been used, but in your book (paragraph: $\mathrm{p} K_{\mathrm{a}}$ determination) is used $\mathrm{KCl}$. I have already noticed that SDS and $\mathrm{KCl}$ can't put together to the solution, so I'm little worried that maybe different salts (same 
concentration) can influence solubility of some compounds. [This is] one more thing which is not yet clear for me. If I use buffer with fixed ionic strength, then final ionic strength for solubility assay will be very different for different compounds. Can it influence the solubility value? Is it better firstly estimate solubility value and then use this value for ionic strength calculation to obtain final ionic strength value near to $0.15 \mathrm{M}$ ? Or it is not a problem that for different compounds ionic strength will be different?

A1: For solubility measurement, $\mathrm{NaCl}$ seems to be popular. (We have seen the $\mathrm{K}^{+}+\mathrm{SDS}$ precipitation, too.) Ideally, you want the final solution to be mimicking the physiological ionic strength, $0.15 \mathrm{M}$. But, sample and buffer will alter that value. A simulation using some program (e.g., $p \mathrm{DISOL-X)}$ would be helpful. When studying salts, it is not easy to keep the ionic strength at the physiological level, due to the excessive sample added.

A2: We do not use extra salt to keep the ionic strength constant.

Q: Why should wetting be a problem in sonic bath? If compound wet very easily, then it should dissolve quickly, because compound will be in contact to water. And when compound is poorly wettable, then sonic should help to wet the compound, because then molecules will move more quickly than usual stirring experiment. The idea to use ultrasonic bath feels more easily performable than construct some device, where I can mix many samples same time. I think that if I could use ultrasonic bath, then I wouldn't need any stirrer in the solution, which will make the system simpler and no extra thing will be in solution, which should be good thing.

A1: If a compound does not wet very well, it just floats on the vibrating water surface, just like a feather from a duck, especially one coated with soot. I think just a single drop of methanol on top of the crystals would cause them to wet (and dissolve), before the buffer is added. I think the main concern about sonic bath is that it may lead to (superstable) supersaturation effects. Perhaps others have experience with this?

Q: I agree that [it is] better [to] predict solubility before experiment and at least know something about the compound. I will try to perform the experiment as you suggest: first add a certain amount compound, and if needed, then add more by weighting. Is it possible to get access to $p D I S O L-X$ software and license? I think it is important to use this program before experiment, because this will make easier to perform correct solutions quicker. This is certain that I will avoid DMSO, because I wish to measure only water solubility, not solubility for mixture (water and DMSO).

A1: Heath Scientific (UK) is the distributor in the EU: Steve.Stones@heathscientific.com. A 30-day FREE trial is available.

Q: I forgot that I have this poster printout. I analyzed her data and I found that maybe it is better not filter at all, because sedimentation will give usually higher solubility value than filtration. Is it reasonable to do this? I think filtration will be only necessary for compounds which have problems with sedimentation.

A1: It depends on the type of compound. Some compounds convert to an oil after the crystals are added to water. Most oils don't sediment very well. Some surface-active compounds form colloid suspensions, which can be lyophilic (e.g., gelatin, ranitidine, guanosine), lyophobic (e.g., some nanoparticles), or associated (e.g., "LLPS" - liquid-liquid phase separated, like some phenothiazines - cf., Lynne Taylor's work at Purdue University) where ultracentrifugation is recommended for separation. In such cases, it may be useful to filter carefully first, and then ultracentrifuge.

Q: About buffer: is it correct to use different buffer system at different pHs? Or it is better to use the same composition buffer for the different $\mathrm{pH}$ values, because then all effects and interactions between compound and buffer components should be same. I agree that ionic strength should be controlled and if I'm not wrong then every $\mathrm{pH}$ should be same ionic strength. It means that best buffer should be such which has constant ionic strength over $\mathrm{pH}$ range or then at different buffers for different $\mathrm{pH}$-s should have same ionic strength. Is it correct? So I will not add any extra salt to buffer, since the compound contributions will usually increase ionic strength sufficiently. Although for some low soluble compounds it will be important, because then not enough compound will be in solution. I think $p D I S O L-X$ will be again the solution for this problem. 
A1: This is a tough but good question. I have done a lot of simulation calculations on a variety of drug substance solutions, to see at which $\mathrm{pH}$ the buffer capacity is low and in need of external boosting. It is not practical to keep the ionic strength exactly constant, and at $0.15 \mathrm{M}$. The $p$ DISOL-X will correct the equilibrium constants and the $\mathrm{pH}$ scale at individual points to a "reference" ionic strength, e.g., $0.15 \mathrm{M}$. The higher the actual ionic strength is above physiological, the more tenuous are the Stokes-Robinson-Debye-Huckel corrections for activity coefficient changes. About buffers: why do we need to use them at all? Two part answer: (a) In very poorly buffered solution, the tiniest addition of titrant to adjust $\mathrm{pH}$ causes huge changes in $\mathrm{pH}$ (e.g., try getting lots of points in the $\mathrm{pH} 3-9$ region in the titration of carbonate-free distilled water with $0.5 \mathrm{M} \mathrm{NaOH}$ ! - not so easy). Also, if the ionic strength is very low in the poorly buffered solution, then the $\mathrm{pH}$ electrode experiences "junction potential" shifts, leading to uncertainty in $\mathrm{pH}$ measurement. The addition of buffer in that steep slope region allows controlled additions of $\mathrm{pH}$ modifier (i.e., "titrant"). How much buffer? - no more than enough to do the job. That depends on the quality and resolution of the liquid dispensing devise. If I recall correctly, the $p I O N$ dispensers can added $30 \mathrm{~nL}$ minimum volumes. My guess is that in most cases, 5-10 mM buffer is enough. Don't forget, the drug itself can serve as a buffer. (b) if the solution is not adequately buffered, then reading of $\mathrm{pH}$ becomes uncertain, and also, trace protogenic impurities can be problematic (like ambient $\mathrm{CO}_{2}$ : it can cause big $\mathrm{pH}$ shifts, leading to uncertainty in the solubility reading for lowsoluble bases with $\mathrm{p} K_{\mathrm{a}}>8$ ). You don't need any extra buffering for $\mathrm{pH}<2$ or $\mathrm{pH}>11$; water does a good job there. To sum up: use "just enough" buffer only in the regions of $\mathrm{pH}$ where buffering is needed. Of course, that all depends on the compound studied. When the contributions from the buffer(s) added are assessed, if the ionic strength is significantly less than $0.15 \mathrm{M}$, then I would added some extra $\mathrm{NaCl}$ to reach $0.15 \mathrm{M}$ total ionic strength. Many people would be surprised how much time I spend planning a measurement for a new drug substance. I am not in favor of "canned formula" use of buffers. Some labs are fond of using the Mcllvaine buffer, which has high citrate and phosphate concentrations. It just makes the data analysis that much more complicated. "Theory guides, experiment decides," or "First think, then measure." - etc., etc. As a final thought, try to avoid buffers that have high UV absorption, if you are doing UV-Vis or HPLC analysis. Some buffers (like phosphate) gum up LC-MS-MS systems.

Q: What criteria did you use in order to make the conclusions at the end of the filter-selection poster? My working hypothesis is that if the value of solubility is unexpectedly low, then filter adsorption of the sample may have been a problem. This is a common assumption.

A2: Yes, this hypothesis is correct. Those conclusions were rather suggestions. It is better to avoid the filtration, but if not possible, we have to select the right filter (hydrophobic for ionized polar species and hydrophilic for unionized lipophilic species); with discarding the first few portions of filtration, etc.

A1: However, can there be a case when high solubility is not a good result? If the solubility assay produced some (not all) very tiny particles and if some were able to pass through the filter pores, then the measured solubility would be unexpectedly high.

A2: It may happen. We think that when we filter a high concentration solution the filter can be saturated more quickly and the error caused by the adsorption is relative less.

A1: Did you have any suggestions about how fast (or slow) to push the syringe plunger through the filter disks?

A2: Good question. We filtered $300 \mu \mathrm{L}$ and $600 \mu \mathrm{L}$ portions, generally quite slowly.

(C)2016 by the authors; licensee IAPC, Zagreb, Croatia. This article is an open-access article distributed under the terms and conditions of the Creative Commons Attribution license (http://creativecommons.org/licenses/by/3.0/) (cc) BY 